Publ. RIMS, Kyoto Univ.

19 (1983), 397 451

\title{
Une Classe d'Opérateurs Différentiels Invariants sur le Groupe de Heisenberg
}

par

N. LOHOUE*

0. Cet article est le premier d'une série où l'auteur se propose d'étudier certains opérateurs différentiels invariants à droite sur les groupes de Lie. La revue de S. Helgason dans [2] est une bonne présentation des résultats existants sur le sujet.

On s'intéressera aux problèmes de résolubilité locale, à la régularité des solutions de $P U=f$. On abordera ces questions le plus souvent par le biais des solutions fondamentales et leur construction explicite.

Les résultats qu'on présente dans ce travail se génèralisent à d'autres groupes de Lie résolubles. Cependant on a préferé présenter d'abord le cas des groupes de Heisenberg plus facilement digeste, puisque la structure algébrique et les répresentations de ces groupes sont simples; les questions difficiles, s'il y en a, sont de nature analytique.

$H_{n}$ désigne le groupe de Heisenberg de dimension $2 n+1 ; X_{j}$, resp. $Y_{j}, 1 \leq j \leq n$ et $Z$ désignent la base canonique de $H_{n}$ avec la relation de commutation habituelle.

$$
\left[X_{i}, Y_{j}\right]=\delta_{i j} Z
$$

On étudiera les opérateurs sur $H_{n}$ de la forme:

$$
P(D)=\sum_{I_{K}, r} a_{I_{K}, r} Y^{I_{K}} X^{I_{K}} Z^{r}
$$

où $I_{K}$ est un multi-indice et $a_{I_{K}, r}$ sont des constantes. En fait on peut prouver des théorèmes avec de $a_{I_{K}, r}$ non constants.

On obtiendra des théorèmes d'existence de solutions fondamentales,

Communiqué par S. Matsuura, le 6 mai, 1981.

* 30 Bld Pasteur, 94260 Fresnes, France.

Ce travail a été achevé et dactylographié au Département de Mathématiques de l'Université de Maryland, College Park, MD 20742 USA. 
d'où la résolubilité locale, l'existence et le contrôle des solutions de $P U$ $=f$.

1. Enoncés des Résultats. Les définitions précises des opérateurs $F_{\delta, \varepsilon}, H_{\delta, \varepsilon}^{\prime}$ sont exposées à la page 408. Dans la première partie du travail nous prouverons l'énoncé suivant.

Théorème I. Soit $P=\sum_{I_{K}, r} a_{I_{K}, r} Y^{I_{K}} X^{I_{K}} Z^{r}$. On suppose que les polynômes suivants:

$$
q_{\alpha}(\lambda)=\sum_{\alpha \leq I_{K} ; r} C_{I_{K}}^{\alpha} a_{I_{K}, r}(-i \lambda)^{r}(-\lambda)^{\left|I_{K}\right|}
$$

n'ont pas de zéros communs. $\quad\left\{C_{I_{K}}^{\alpha}\right.$ sont des constantes dont la dèfinition est à la page 405.

$1^{\circ}$. Soit $P^{*}$ l'adjoint de $P$. Alors il existe deux distributions $E_{\delta, \varepsilon}$ et $E_{\delta, \varepsilon}^{*}$ dépendant de deux paramètres $\varepsilon \in \boldsymbol{R}^{n}, 0<\delta<1 / 4 n, 2 \delta<\varepsilon_{j}+\delta<1$ dont l'une est solution fondamentale de $P$, l'autre celle de $P^{*}$.

De plus

$$
E_{\delta, \varepsilon}=F_{\delta, \varepsilon} * E_{\delta, \varepsilon}^{0} * H_{\delta, \varepsilon}^{\prime}
$$

où $E_{\delta, \varepsilon}^{0}$ est un opérateur de convolution borné sur $L^{2}\left(H_{n}\right)$.

$2^{\circ}$. $P$ et $P^{*}$ sont localement résolubles: soit $\Omega$ un ouvert relativement compact, soit $f \in C_{c}^{\infty}(\Omega)$, il existe une fonction $W \in C(\Omega)$ telle que $P W$ ou $P^{*} W$ coïncide avec $f$ sur $\Omega$.

$P$ et $P^{*}$ sont surjectifs sur $C^{\infty}\left(H_{n}\right)$.

On déduira du théorème un corrolaire qui donne des estimations globales des solutions de $P U=f$.

Nous renvoyons les énoncés et preuves d'une forme un peu plus précise du théorème I et des corollaires qu'on en déduit à la page 408.

On peut signaler tout de suite que les solutions fondamentales ainsi construites ne sont ni explicites, ni régularisantes. Si l'on veut avoir des solutions régulières de $P U=f$, il faut imposer des conditions sur $P$; sinon il faudra des conditions draconiennes sur $f$. 
On verra (page 406) qu'à l'opérateur $P=\sum_{I_{K}, r} a_{I_{K}, \gamma} Y^{I_{K}} X^{I_{K}} Z^{r}$ on peut associer un polynôme $Q(\xi, \lambda),(\xi, \lambda) \in \mathbb{R}^{n+1}$. Les solutions fondamentales qu'on veut décrire dépendent de ce polynôme.

L'opérateur suivant, dans $H_{3}$ est assez parlant:

$$
P=-Y^{2} X^{2}+Y X Z+R(Z)
$$

où $R$ est un polynôme.

Si $R(i \lambda)$ est réél et $R(i \lambda) \geq 1+\lambda^{2} / 3, P$ aura une solution fondamentale qui est un opérateur de convolution borné sur $L^{2}\left(H_{n}\right)$.

De plus si $R(i \lambda) \geq 1+\lambda^{4}+\lambda^{2} / 3$, on aura une formule pour la solution fondamentale qui est donnée par le théorème IIa ci-dessous.

Par contre si $0 \leq R(i \lambda) \leq \lambda^{2} / 4$, le polynôme associé à $P$ qui vaut ici $Q(\xi, \lambda)=\lambda^{2} \xi^{2}+R(i \lambda)$ aura des zéros $Z(\lambda)$ avec $0<\operatorname{Im} Z(\lambda)<1 / 2$, on peut encore trouver une solution fondamentale, moins bonne que les deux précédentes mais pas aussi mauvaise que la solution fondamentale du théorème I (voir le théorème III).

Mais la situation se gâte plus avec l'opérateur $P=Y_{1} X_{1}+i Y_{2} X_{2}+a_{0}$ dans $H_{5}$ dont le polynôme associé est $Q(\xi, \lambda)=-i \lambda \xi_{1}+\lambda \xi_{2}+a_{0}$. Si $a_{0}$ est réel, pour chaque $\lambda$ la variété des zéros de $Q$ rencontre la bande $0 \leq \operatorname{Im} \xi_{i} \leq 2$.

On ne pourra ni appliquer le théorème II, ni le théorème III à cet opérateur.

Pour terminer ce paragraphe signalons comme on le verra au cours des démontrations que la régularité des solutions fondamentales se mesure par l'absence des zéros des polynômes associés dans la bande $0 \leq \operatorname{Im} \xi_{i}$ $\leq 1 / 2$ de $\mathbb{C}^{n}$.

Le second énoncé que nous prouverons donne des formules explicites des solutions fondamentales pour une classe d'opérateurs.

Ce résultat s'applique aux opérateurs de la forme:

$$
P=\sum_{|\alpha| \leq 2 m} a_{\alpha} P^{\alpha}+R(Z)
$$

pour un bon choix des $a_{\alpha}$ et de polynôme $R$ voir (pages 438 et 439).

Pour énoncer ce résultat, nous aurons besoin de quelques définitions.

Définitions. Soient $I \subset J=\{1 ; \cdots ; n\}$ et $I^{c}$ son complémentaire. On note $\mathbf{1}_{I}$ la fonction caractéristique du sous-ensemble $\Gamma_{I}=\left\{x \in \mathbb{R}^{n}\right.$, 
tel que $x_{i}>0, \forall i \in I$ et $x_{i}<0$, si $\left.i \notin I\right\}$. On note $\mathbb{1}_{I}^{+}$la fonction caractéristique de $v \in \mathbb{R}^{|I|}$ telle que $v_{i} \geq 0$ pour tout $i \in I$; Pour les notations $\left(x_{I}, x_{I c}\right)$ et $\log _{I}((U+x) / U)$ voir page 425.

On prouvera alors l'énoncé suivant:

Théorème II. Soit $P=\sum_{I_{K}, r} a^{I_{K}, r} Y^{I_{K}} X^{I_{K}} Z^{r}$ et soit $Q$ le polynôme associé. On suppose dans les deux cas ci-dessous que pour $\lambda$ fixé $|Q(\xi, \lambda)| \geq c>0$ dans le tube $0 \leq \operatorname{Im} \xi_{i} \leq 1 / 2$.

a) On suppose aussi que $\int_{\boldsymbol{R}^{n+1}}|\lambda|^{n}\left|Q^{-1}(\xi, \lambda)\right| d \xi d \lambda<\infty$. Soit H la transformée de Fourier de la fonction $\left.|\lambda|\right|^{n} Q^{-1}(\xi, \lambda)$. Alors

$$
\begin{aligned}
E= & \lim _{\varepsilon \rightarrow 0} \sum_{I \subset J} \int_{\left|u_{i}\right| \leq 1 / \varepsilon} \mathbb{1}_{I}^{+}\left(U_{I}\right) \mathbf{1}_{I c}^{+}\left(-U_{I c}\right) \mathbb{1}_{I}(U+x) \\
& \times H\left[\log _{I}\left(\frac{U+x}{U}\right), Y \cdot(U+x)-z\right] \frac{d U}{U}
\end{aligned}
$$

existe au sens des distributions; c'est une solution fondamentale de $P: P E=I_{d}$.

b) Soit $\mu$ le dégrè en $\xi$ du polynôme $Q(\xi, \lambda)$; on suppose qu’il existe un polynôme $q(\lambda)$ en $\lambda$, sans zéro réel tel que

$$
|Q(\xi+i \eta, \lambda)| \geq c\left(\|\lambda \xi\|^{\mu}+|q(\lambda)|\right] \quad 0 \leq \eta_{i} \leq 1 / 2 .
$$

Pour $p$ assez grand on note $H_{p}$ la transformée de Fourier de la fonction $Q^{-1}(\xi, \lambda)|\lambda|^{n}\left(1+\lambda^{2 p}\right)^{-1}$. Alors

$$
\begin{aligned}
& \lim _{\varepsilon \rightarrow 0} \sum_{I \subset J} \int_{\left|u_{i}\right| \leq 1 / \varepsilon} \mathbb{1}_{I}^{+}\left(u_{I}\right) \mathbf{1}_{I^{c}}^{+}\left(-U_{I^{c}}\right) \mathbf{1}_{I}(u+x) \\
& \quad \times H_{p}\left[\log _{I}\left(\frac{U+x}{U}\right), Y \cdot(U+x)-z\right] \frac{d U}{U}=E_{0}
\end{aligned}
$$

existe au sens des distributions et $E=\left[I_{d}-\left(\frac{\partial}{\partial z}\right)^{2 p}\right] E_{0}$ est une solution fondamentale de $P$ : $P E=I_{d}$.

Dans le dernière partie, on s'intéresse à une classe d'opérateurs différentiels invariants déduite de celles qu'on a étudié dans les deux premières. On donnera une condition suffisante d'existence de solutions fondamentales 
pour cette classe.

Plus précisément, pour tout $\omega \in \boldsymbol{R}^{n}$, on considère l'opérateur:

$$
\begin{aligned}
P^{\omega}=T(Y+\omega Z, Z) & \sum_{I_{K}, r} a_{I_{K}, r}(Y+\omega z)^{I_{K}} X^{I_{K}} Z^{r} \\
& +\sum_{\substack{|p|+|s| \leq m \\
r \leq p}} b_{p, r, s}(Y+\omega Z)^{p} X^{r} Z^{s}
\end{aligned}
$$

où $Y+\omega Z=\left(Y_{1}+\omega_{1} Z ; \cdots ; Y_{n}+\omega_{n} Z\right) ; T$ est un polynôme en $Y_{i}+\omega_{i} Z$ et $Z$; il existe un entier $\beta>0$ tel que le degré de $\widehat{T}(i \lambda \xi, \lambda)$ transformé de Fourier de $T$, comme polynôme en $(i \lambda \xi)$, soit égal à $m+\beta$.

On suppose que $\operatorname{Max}\left\{\left|I_{K}\right|, a_{I_{K}, r} \neq 0\right\}=2 \mu$ où $\mu$ est un entier et que $\left|r_{0}\right|=\operatorname{Max}\left\{|r|, b_{p, r, s} \neq 0\right\}<2 \mu$. A l'opérateur $P_{0}=\sum_{I_{K, r}} a_{I_{K}, r} Y^{I_{K}} X^{I_{K}} Z^{r}$ correspond comme on l'a déjà signalé (page 399) un polynôme $Q(\xi, \lambda)$ $=\sum_{\alpha} q_{\alpha}(\lambda) \xi^{\alpha}$.

Par la suite on aura besoin de la fonction suivante:

$$
q_{2}(\lambda)=\sum_{j=1}^{2 \mu}\left(\sum_{j<|\alpha| \leq 2 \mu}\left|q_{\alpha}(\lambda)\right|\right)|\lambda|^{j}+\sum_{j=1}^{r_{0}}|\lambda|^{j} .
$$

Pour la définition de l'opérateur $H^{\theta}$ et la norme \|\|$_{2, \theta}$, voir la page 449. On prouvera l'énoncé suivant.

Théorème III. On suppose qu'il existe

i) $0<\varepsilon_{j}, \varepsilon_{j}^{\prime}<1$ et une fonction $\theta: \mathbb{R} \rightarrow \mathbb{R}^{n}$, mesurable $\varepsilon_{j}<\theta_{j}<2-\varepsilon_{j}^{\prime}$

ii) $0<e_{1}, e_{2}, 0 \leq \eta$, constantes

iii) deux polynômes d'une variable $q_{0}, q>1$ tels que:

$$
\begin{aligned}
& \left|Q_{\lambda}(\xi+i y)\right| \geq e_{1}\left[\|\lambda(\xi+i y)\|^{2}+q(\lambda)\right]^{\mu} \text { si }\|\theta(\lambda) / 2-y\| \leq \eta \\
& |\widehat{T}(i \lambda \xi, \lambda)| \geq e_{2}\left[\|\lambda \xi\|^{2}+q_{0}^{2}\right]^{(m+\beta) / 2} .
\end{aligned}
$$

Soient

$$
C(\lambda)=\left\{\begin{array}{lll}
\left|q_{0}(\lambda)\right|^{-\beta} \sum_{p, r, s}\left|b_{p, r, s}\right||\lambda|^{s} & \text { si } & |\lambda| \leq 1 \\
\left|q_{0}(\lambda)\right|^{-\beta} \sum_{p, r, s}\left|b_{p, r, s}\right||\lambda|^{s+\left|r_{0}\right|} & \text { si } & |\lambda|>1
\end{array}\right.
$$

(on suppose que $C(\lambda)$ est bornée par une constante).

$$
C_{1}(\lambda, T)=\sum_{\substack{\alpha \neq 0 \\ r}} \frac{\left|t_{\alpha, r}\right||\lambda|^{r}}{\left|q_{0}(\lambda)^{m+\beta-|\alpha|}\right|} \text { où } \widehat{T}(i \lambda \xi, \lambda)=\sum_{\alpha, r} t_{\alpha, r}(-i \lambda \xi)^{\alpha}(-i \lambda)^{r} \text {. }
$$


Alors il existe une constante absolue $C\left(\varepsilon, \varepsilon^{\prime}, \theta, \eta, \mu, m\right)$ et un entier $L_{T}$ telle que $P$ possède une solution fondamentale $E$ si:

i) $\sup _{|\lambda| \geq 1}|q(\lambda)|^{-1 / 2} C_{1}(\lambda, T)^{L_{T}} C(\lambda)\left\{\left[q_{2}(\lambda)\right]^{2 \mu(n+1)}+1\right\}\left\{|\lambda|^{-2 \mu+1}+|\lambda|^{-(n+1)}\right.$ $\left.+|\lambda|^{-n-2 \mu+1}\right\} \leq C\left(\varepsilon, \varepsilon^{\prime}, \theta, \eta, \mu, m\right)$

ii) $\sup _{|\lambda| \geq 1} C(\lambda)|q(\lambda)|^{-1 / 2}\left(|\lambda|^{-2 \mu+1}+\left.\left|\lambda_{1}^{\mid-(n+1)}+\right| \lambda\right|^{-n-2 \mu+1}\right)$

$$
\leq C\left(\delta, \delta^{\prime}, \theta, \eta, \mu, m\right)
$$

iii) De plus on a l'estimation

$$
\left\|H^{-\theta} E f\right\|_{L^{2\left(H_{n}\right)}} \leq C\|f\|_{2, r_{\theta}} .
$$

On dèduit évidemment des résultats de résolubilité locale de ce théorème.

Ce travail a été entrepris pendant un séjour au département de Mathématiques de l'Université McGill à Montréal. Il n'aurait peut-être pas été conçu sans l'atmosphère exceptionnellement amicale de "Burnside Hall." Nous tenons à remercier ceux qui y ont contribué de près où de loin.

Nous avons aussi profité d'une invitation au département de Mathématiques de l'Université de Maryland pour faire des mises au point des théorèmes I et III. Nous tenons à remercier Profs. R. Johnson et W: Kirwan pour ce séjour agréable "il fait beau en octobre à College Park" et fructueux.

Nos égards vont aussi à Mrs. Berta V. Casanova. Elle a assuré la frappe de ce texte avec beaucoup d'habileté et de gentillese, elle a travaillé contre la montre.

\section{$\S 1$. Quelques Notations et Définitions}

On note $H_{n}$ le groupe de Heisenberg de dimension $2 n+1$; un point générique $h \in H_{n}$ peut se décrire par trois coordonées $h=(x, y, z), x \in \boldsymbol{R}^{n}$, $y \in \mathbb{R}^{n}, z \in \boldsymbol{R}$.

On rappelle que le produit $h h^{\prime}$ de deux élèments du groupe est donné par la formule: $h h^{\prime}=(x, y, z)\left(x^{\prime}, y^{\prime}, z^{\prime}\right)=\left(x+x^{\prime}, y+y^{\prime}, z+z^{\prime}\right.$ $\left.+x y^{\prime}\right)$. 
On note $X_{j}$, resp $Y_{j}, 1 \leq j \leq n$ et $Z$ le vecteur de l'algèbre de Lie de $H_{n}$ tel que

$$
\exp X_{j}=\left(e_{j}, 0,0\right) ; \exp Y_{j}=\left(0, e_{j}, 0\right) ; \exp Z=(0,0,1) \text {. }
$$

Où l'on a désigné par exp l'application exponentielle de l'algèbre de Lie de $H_{n}$ sur lui-même. Alors on a les relations de commutation habituelle:

$$
\left[X_{i}, Y_{j}\right]=\delta_{i j} Z \text {. }
$$

Le groupe $H_{n}$ posséde une série de représentations fondamentales définies comme suit. Soit $f: \mathbb{R}^{n} \rightarrow \mathbb{C}$ une fonction de classe $C_{c}^{\infty}\left(\mathbb{R}^{n}\right)$ et soit $h=(x, y, z)$ un point de $H_{n}$, on pose:

$$
\Pi_{\lambda}(h) f(\xi)=\exp i \lambda(Y \cdot \xi-Z) f(\xi-X) .
$$

Pour tout $\lambda$ réel non nul, on obtient ainsi une représentation unitaire irréductible de $H_{n}$ sur l'espace $L^{2}\left(\boldsymbol{R}^{n}\right)$ des fonctions complexes de carré intégrables sur l'espace euclidien $\mathbb{R}^{n}$ pour la mesure de Lebesgue.

Soit $\varphi: H_{n} \rightarrow C$ une fonction $C_{c}^{\infty}\left(H_{n}\right)$ on définit $\widehat{\varphi}(\lambda)$ comme l'opérateur:

$$
\widehat{\varphi}(\lambda)=\int_{H_{n}} \Pi_{\lambda}(h) \varphi(h) d h
$$

Ainsi on a la formule de Plancherel:

$$
\left.\int_{H_{n}}|\varphi(x)|^{2} d x=\int_{-\infty}^{+\infty} \operatorname{Tr} \mid \widehat{\varphi}(\lambda) \hat{\varphi}^{*}(\lambda)\right]|\lambda|^{n} d \lambda .
$$

Dans cette formule $\operatorname{Tr} A$ désigne la trace de l'opérateur $A$ et $A^{*}$ son adjoint.

La différentielle de $\Pi_{\lambda}$ agit sur les vecteurs précédents de la façon suivante:

$$
\begin{array}{ll}
d \Pi_{\lambda}\left(X_{j}\right)=-\frac{\partial}{\partial \xi_{j}} & 1 \leq j \leq n \\
d \Pi_{\lambda}\left(Y_{j}\right)=i \lambda \xi_{j} & 1 \leq j \leq n \\
d \Pi_{\lambda}(Z)=-i \lambda . &
\end{array}
$$

On s'intéresse à la classe d'opérateurs différentiels:

$$
P(h, D)=\sum_{\substack{0 \leq i_{j} \leq m_{j} \\ 1 \leq j \leq n}} a_{i_{1}, \cdots, i_{n+1}}(h) Y_{1}^{i_{1}} X_{1}^{i_{1}} \ldots Y_{n}^{i_{n}} X_{n}^{i_{n}} Z^{i_{n+1}} .
$$

Dans un premier temps on s'intéressera au cas où les coefficients sont 
constants

$$
P(h, D)=\sum_{\substack{0 \leq i_{j} \leq m_{j} \\ 1 \leq j \leq n}} a_{i_{1}, \ldots, i_{n+1}} Y_{1}^{i_{1}} X_{1}^{i_{1}} \ldots Y_{n}^{i_{n}} X_{n}^{i_{n}} Z^{i_{n+1}} .
$$

Pour simplifier les écritures, on notera cet opérateur par:

$$
P(D)=\sum_{I_{K}, r} a_{I_{K}, r} Y^{I_{K}} X^{I_{K}} Z^{r}
$$

Dans cette notation $I_{K}$ est un esemble d'indices entiers indexés par une partie $K$ de $J=(1, \cdots, n): I_{K}=\left\{i_{k_{1}}, \cdots, i_{k_{s}}\right\}$ où $k_{j} \in K \subset J$.

$Y^{I_{K}}$, resp $X^{I_{K}}$, désigne l'opérateur différentiel,

$$
Y^{I_{K}}=\prod_{i_{k} \in I_{K}} Y_{k}^{i_{k}}, \quad \operatorname{resp} . X^{I_{K}}=\prod_{i_{k} \in I_{K}} X_{k}^{i_{k}}
$$

On peut ramener, comme nous le verrons dans les pages qui suivent, l'étude de ces opérateurs à celle des opérateurs différentiels à coèfficients constants.

Pour introduire ces opérateurs, nous aurons besoin de quelques notations.

\section{Notations.}

$1^{\circ}$. Si $x \in \boldsymbol{R}^{n}, x_{I}$ désigne le point: $x_{I}=\left\{x_{i_{1}}, \cdots, x_{i_{r}}\right\}$ de $\boldsymbol{R}^{|I|}$ où l'on a noté $|I|$ le cardinal de $I$
a) $i_{j} \in I$
b) $i_{j} \leq i_{j+1}$
c) $x_{i_{j}}$ est la $i_{j}$ éme composante de $x$.
d) $|I|$ est égal à $r$.

Ainsi chaque point $x$ de $\boldsymbol{R}^{n}$ s'écrit $x=\left\{x_{I}, x_{I c}\right\}$

$2^{\circ}$ Soient $I_{K}=\left\{i_{1}, \cdots, i_{r}\right\}$ où $i_{k} \leq i_{k+1}$ et $K \subset J$ on pose, pour $\delta \in \boldsymbol{R}$

$$
D^{I_{K}}=\prod_{i_{k} \in I_{K}}\left(-i \frac{\partial}{\partial \xi_{k}}\right)^{i_{k}} \quad\left|X_{I}\right|^{\delta}=\prod_{i \in I}\left|x_{i}\right|^{\delta} .
$$

Soit $t \in \boldsymbol{R}^{n}$ on pose:

$$
\begin{aligned}
e^{t_{I_{K}}} & =\left(e^{t_{i_{1}}}, \cdots, e^{t_{i_{r}}}\right) \\
e^{t_{I_{K}^{c}}} & =\left(e^{t_{j_{1}}}, \cdots, e^{t_{i_{n-r}}}\right)
\end{aligned}
$$

où l'on a ordonné $I_{K}^{c}$ comme $I_{K}$. 
On définit alors un difféomorphisme $\Psi_{I}$ de $\mathbb{R}^{n}$ dans $\Gamma_{I}$ par la relation: pour la définition de $\Gamma_{I}$ (voir page 399)

$$
\Psi_{I}(t)=\left(e^{t_{I}},-e^{t_{I c}}\right) .
$$

Pour toute fonction $U: \mathbb{R}^{n} \rightarrow \boldsymbol{C}$, on note $U_{I}$ sa restriction à $\Gamma_{I}$ et $\widetilde{U}_{I}$ la fonction:

$$
\widetilde{U}_{I}=U_{I} \circ \Psi_{I}
$$

$4^{\circ}$ Pour tout $\alpha \in \mathbb{R}^{n}$ on note $L^{2}\left(\mathbb{R}^{n}, e^{\alpha \cdot x} d x\right)$ l'espace de Hilbert des fonctions $f: \mathbb{R}^{n} \rightarrow \mathbb{C}$ telles que:

$$
\|f\|_{2, \alpha}^{2}=\int_{\boldsymbol{R}^{n}}|f(x)|^{2} e^{\alpha \cdot x} d x<\infty
$$

$L^{2}\left(\boldsymbol{R}^{n}, e^{\alpha \cdot x} d x\right)$ est muni de la norme $\|f\|_{2, \alpha}$.

$5^{\circ}$ Pout tout $\lambda \in \mathbb{R}, \Pi_{\lambda}(P)=P_{\lambda}$ désignera la valeur de la transformée de Fourier de $P$ au point $\Pi_{\lambda}$.

On veut établir la proposition suivante grâce à laquelle l'étude de l'opérateur $P$ précédent se ramènera à l'étude d'opérateurs à coefficients constants.

Proposition 1. Pour tout $\lambda \neq 0$, il existe un opérateur différentiel sur $\mathbb{R}^{n}$ à coefficients constants $Q_{\lambda}(D)$ tel que pour tout couple $(U, f)$ de fonctions $C_{c}^{\infty}\left(\mathbb{R}^{n}\right)$ la relation

$$
P_{\lambda} U=f
$$

a lieu si et seulement si pour toute partie finie $I \subset J$

$$
Q_{\lambda}(D) \widetilde{U}_{I}=\tilde{f}_{I} \text {. }
$$

Preuve de la Proposition. Pour prouver cette proposition nous aurons besoin de fixer quelques notations. Soit $\left(i_{j}, k_{j}\right)$ un couple d'entiers positifs, $0 \leq k_{j} \leq i_{j}$, on note $C_{i_{j}}^{k_{j}}$ le $k_{j}^{\text {ème }}$ coefficient du polynôme $\prod_{r=0}^{i_{j}}(\xi-r)$.

Si $I_{r}$ et $I_{r}^{\prime}$ sont deux ensembles ordonnés d'indices: $I_{r}=\left(i_{1}, \cdots, i_{r}\right)$ et $I_{r}^{\prime}=\left(i_{1}^{\prime}, \cdots, i_{r}^{\prime}\right)$ tels que $0 \leq i_{j}^{\prime} \leq i_{j}$ pour tout $j$, on posera:

$$
C_{I_{r}}^{I_{r}^{\prime}}=\prod_{i_{j} \in I_{r}} C_{i_{j}}^{i_{j}^{\prime}}
$$


Considérons l'équation différentielle ordinaire d'Euler d'ordre $k$ sur $\boldsymbol{R}$ : $x^{k} \frac{d^{k}}{d x^{k}}$ où $k>0$ est un entier:

Soient $U$ et $f$ deux fonctions de classe $C_{c}^{\infty}(\boldsymbol{R})$. On pose:

$$
\begin{array}{ll}
\widetilde{U}_{1}(z)=U\left(e^{z}\right) ; & \widetilde{U}_{2}(z)=U\left(-e^{z}\right) \\
\tilde{f}_{1}(z)=f\left(e^{z}\right) ; & \tilde{f}_{2}(z)=f\left(-e^{z}\right) .
\end{array}
$$

Alors:

$$
\begin{aligned}
{\left[x^{k} \frac{d^{k}}{d x^{k}}(U)\right]\left(e^{z}\right) } & =\left[\prod_{r=0}^{k-1}\left(\frac{d}{d z}-r\right)\right] \widetilde{U}_{1}(z) \\
& =\prod_{r=0}^{k} C_{k}^{r}\left[\left(\frac{d}{d z}\right)^{k-r} \widetilde{U}_{1}\right](z) .
\end{aligned}
$$

On a une égalité analogue pour $\widetilde{U}_{2}$.

D'une part:

$$
P_{\lambda}(\xi, D)=\sum_{I_{K}}\left(\sum_{\gamma} a_{I_{K}, \gamma}(-i \lambda)^{r}(-\lambda)^{\left|I_{K}\right|}\right) \xi^{I_{K}} D^{I_{K}} .
$$

D'autre part si $U: \boldsymbol{R}^{n} \rightarrow \boldsymbol{C}$ est une fonction de classe $C_{c}^{\infty}, I \subset J$ et $\widetilde{U}_{I}$ la fonction correspondante à $U$ définie au paragraphe précèdent (page 405), d'après ce qui a été dit ci-dessus,

$$
\begin{aligned}
& \left(P_{\lambda}(\xi, D) U\right) \circ \phi_{I} \\
& \quad=\sum_{I_{K}}\left(\sum_{\gamma} a_{I_{K}, r}(-i \lambda)^{r}(-\lambda)^{\left|I_{K}\right|}\right) \prod_{i_{k} \in I_{K}}(-i)^{i_{k}} \sum_{i_{k}^{\prime}=0}^{i_{k}} C_{i_{k}}^{i_{k}^{\prime}} D^{i_{k}-i_{k}^{\prime}} \widetilde{U}_{I} \\
& \quad=Q_{\lambda}(D) \widetilde{U}_{I} .
\end{aligned}
$$

Par conséquent $P_{\lambda}(\xi, D) U=f$ si et seulement pour tour $I \subset J, Q_{\lambda}(D) \widetilde{U}_{I}$ $=\tilde{f}_{I}$.

Avant d'attaquer la preuve du théorème I faisons la remarque suivante:

Remarque. Pour tout opérateur différentiel à coefficients constants $Q(D)$ sur $\boldsymbol{R}^{n}$, il existe un opérateur $P$ sur $H_{n}$ de page 397 tel que l'opérateur $Q_{1}(D)$ associé à $P_{\lambda}$ par la proposition précédente pour la valeur 1 du paramétre soit $Q(D)$.

Cette remarque est facile est établir, nous laissons la preuve au 
lecteur.

Expression du symbole de $Q_{\lambda}(D)$.

Par définition

$$
P_{\lambda}(\xi, D)=\sum_{I_{K}, r} a_{I_{K}, r}(-i \lambda)^{I_{K}+\gamma} \xi^{I_{K}} \frac{\partial^{I_{K}}}{(\partial \xi)^{I_{K}}} .
$$

Par conséquent le symbole de $Q_{\lambda}(D)$ vaut:

$$
Q_{\lambda}(\xi)=\sum_{I_{K}, r} a_{I_{K}, r}(-i \lambda)^{r}(-\lambda)^{\mid I_{K^{\prime}}} \prod_{i_{k} \in I_{K}} \xi_{k}^{i_{k}}+\sum_{1 \leq p_{k} \leq i_{k}-1} C_{p_{k}}^{i_{k}} \xi_{k}^{i_{k}-p_{k}}
$$

Mais

$$
\prod_{i_{k} \in I_{K}}\left(\xi_{k}^{i_{k}}+\sum_{1 \leq p_{k} \leq i_{k}-1} C_{p_{k}}^{i_{k}} \xi_{k}^{i_{k}-p_{k}}\right)=\sum_{1 \leq \alpha_{k} \leq i_{k}}\left(\prod_{i_{k} \in I_{K}} C_{i_{k}-\alpha_{k}}^{i_{k}}\right) \xi^{\alpha}
$$

où l'on convient que $C_{i_{k}}^{i_{k}}=1$.

Soit $C_{I_{K}}^{\alpha}=\prod_{i_{k} \in I_{K}} C_{i_{k}-\alpha_{k}}^{i_{k}}$. Alors :

$$
\begin{aligned}
Q_{\lambda}(\xi) & =\sum_{I_{K}, \gamma} a_{I_{K}, \gamma}(-i \lambda)^{\left|I_{K}\right|} \sum_{0 \leq \alpha \leq I_{K}} C_{I_{K}}^{\alpha} \xi^{\alpha} \\
& =\sum_{\alpha} \xi^{\alpha}\left(\sum_{0 \leq \alpha \leq I_{K}} C_{I_{K}}^{\alpha} a_{I_{K}, r}(-i \lambda)^{\gamma}(-\lambda)^{\left|I_{K}\right|}\right) .
\end{aligned}
$$

\section{$\S$ 2. Preuve du Théorème $\mathbb{I}$}

La preuve de ce théorème nécessite quelques lemmes et des notations.

Notations. Soit $\theta: \mathbb{R} \mapsto \boldsymbol{R}$ une fonction mesurable et soit $I \subset J$. On note $\left|Y_{I}\right|^{\theta}$ l'opérateur, à priori non borné, sur $L^{2}\left(H_{n}\right)$ dont la transformée de Fourier pour chaque $\lambda$ est l'opérateur de multiplication par la fonction $\prod_{i \in I}\left|\xi_{i}\right|^{\theta_{i}(\lambda)}$. Si $\theta \in \mathbb{R}^{n}$, on le considère comme l'application constante de $\mathbb{R}$ dans $\boldsymbol{R}^{n}: \theta_{i}(t)=\theta_{i}$ et $\left|Y_{I}\right|^{\theta}$ aura le sens ci-dessus. De même si $\delta \in \mathbb{R}$, on le considère comme le vecteur $\vec{\delta}=(\delta, \cdots, \delta)$ de $\boldsymbol{R}^{n}$ et $\left|Y_{I}\right|^{\delta}$ est défini comme précédement.

Pour tout $j \in J$, on désignera par $\varphi_{j}^{0}$ la fonction caractéristique de l'intervalle $\left|x_{j}\right|<1$ et $\varphi_{j}^{1}$ la fonction caractéristique des $\left|x_{j}\right|>1$. Soient:

$$
\varphi_{I}^{0}(x)=1_{B}(x) \prod_{i \in I} \varphi_{i}^{0}\left(x_{i}\right) ; \quad \varphi_{I}^{1}(x)=\prod_{i \in I} \varphi_{i}^{1}\left(x_{i}\right) 1_{B}(x)
$$

où $1_{B}$ est la fonction caractéristique des $x \in \mathbb{R}^{n}$ tels que $x_{j} \neq 1$ pour $j \in J$. On note $\Phi_{I}^{0}$, resp $\Phi_{I}^{1}$, l'opérateur de convolution sur $H_{n}$ dont la trans- 
formée de Fourier pour chaque $\lambda$ est l'opérateur de multiplication par $\varphi_{I}^{0}$, resp, $\varphi_{I}^{1}$.

Soient $\varepsilon \in \boldsymbol{R}^{n}, 0 \leq \varepsilon_{i} \leq 1,0<\delta<1 / 4 n$ et $\delta_{0}=(n-\varepsilon \cdot 1+(1-\delta n) / 2) / 2$. On supposera par la suite que: $\forall j ; \varepsilon_{j}+\delta<1, \varepsilon_{j}-\delta>0$. On définit les opérateurs $F_{\delta, \varepsilon}, F_{\delta, \varepsilon}^{\prime}, H_{\delta, \varepsilon}$ et $H_{\delta, \varepsilon}^{\prime}$ par les formules:

$$
\begin{aligned}
& F_{\delta, \varepsilon}=|Z|^{\delta_{0}} * \sum_{I \subset J} \Phi_{I}^{0} *\left|Y_{I}\right|^{(1-\delta-\varepsilon) / 2} * \Phi_{I c}^{1} *\left|Y_{I c}\right|^{(1-\varepsilon+\delta) / 2} \\
& F_{\delta, \varepsilon}^{\prime}=|Z|^{-\delta_{0}} * \sum_{I \subset J} \Phi_{I}^{0} *\left|Y_{I}\right|^{-(1-\delta-\varepsilon) / 2} * \Phi_{I c}^{1} *\left|Y_{I c}\right|^{(1-\varepsilon-\delta) / 2} \\
& H_{\delta, \varepsilon}=|Z|^{\delta_{0}} * \sum_{I C J} \Phi_{I}^{0} *\left|Y_{I}\right|^{(1-\varepsilon+\delta) / 2} * \Phi_{I c}^{1} *\left|Y_{I c}\right|^{(1-\varepsilon-\delta) / 2} \\
& H_{\delta, \varepsilon}^{\prime}=|Z|^{-\delta_{0}} * \sum_{I C J} \Phi_{I}^{0} *\left|Y_{I}\right|^{-(1-\varepsilon+\delta) / 2} * \Phi_{I c}^{1} *\left|Y_{I c}\right|^{-(1-\varepsilon-\delta) / 2}
\end{aligned}
$$

Remarquons tout de suite que $F_{\delta, \varepsilon}^{\prime}$, resp $H_{\delta, \varepsilon}^{\prime}$, ainsi défini est l'inverse de $F_{\delta, \varepsilon}$, resp $H_{\delta, \varepsilon}$. On prouvera l'énoncé suivant dont dé coulera le théorème I.

Théorème $\mathbb{I}^{\prime}$. Soit $P=\sum_{I_{K}, r} a_{I_{K}} Y^{I_{K}} X^{I_{K}} Z^{r}$; on suppose que les polynômes suivants n'ont pas de zéros communs: $q_{\alpha}(\lambda)=\sum_{I_{K}, r} \sum_{0 \leq \alpha \leq I_{K}} C_{I_{K}}^{\alpha} a_{I_{K}, r}$ $\times(-i \lambda)^{r}(-\lambda)^{\left|I_{\boldsymbol{K}}\right|}$. Soient $\delta$ et $\varepsilon$ comme ci-dessus, alors il existe un opérateur $E_{\delta, \varepsilon}^{0}$ de convolution de $L^{2}\left(H_{n}\right)$ telque la distribution $E_{\delta, \varepsilon}=$ $F_{\delta, \varepsilon} * E_{\delta, \varepsilon}^{0} * H_{\delta, \varepsilon}$ vérifie l'égalité: $P E_{\delta, \varepsilon}=H_{\delta, \varepsilon}$.

On peut prouver un énoncé analogue même si $\varepsilon=0$; ceci conduirait au corollaire 2 ci-dessous. La preuve du théorème $I^{\prime}$ repose sur une proposition et une série de lemmes dont les énoncés nécessitent quelques notations.

Notations. $1^{\circ}$ Pour tout $x \in \mathbb{R}^{n}$, on note $\|\log x\|$ la quantité $\|\log x\|=\left(\sum_{i=1}^{n}\left(\log \mid x_{i i}\right)^{2}\right)^{1 / 2}$. Si $\delta \in \boldsymbol{R}, \varepsilon \in \mathbb{R}^{n}, L_{\delta, \varepsilon}^{2}\left(\mathbb{R}^{n}\right)$ désignera l'espace des fonctions sur $\boldsymbol{R}^{n}$ de carré sommables pour la mesure $\left|X_{J}\right|^{\varepsilon-1} e^{2 \delta\|\log x\|} d x$.

On note aussi $F_{\delta, \varepsilon}$ l'espace des fonctions de carré sommables pour la mesure $e^{\varepsilon \cdot x} e^{2 \delta\|x\|}$

$2^{\circ}$ Soit $P(c, D)=\sum_{|\alpha| \leq m} C_{\alpha} D^{\alpha}$ un opérateur différential sur $\mathbb{R}^{n}$ à coefficients constants: On pose: 


$$
|c|=\left(\sum_{|\alpha| \leq m}\left|C_{\alpha}\right|^{2}\right)^{1 / 2}
$$

On note aussi $N(m, n)$ la dimension de l'espace vectoriel des polynômes en $n$ variables de degré $m$ et $k=\left(k_{1}, \cdots, k_{\omega}\right)$ un point de $\mathbb{R}^{\omega}$ où $\omega=$ $2 N(n, m)$, à coordonnées entières.

On prouvera la:

Proposition 2. Soient $0<\delta<1 / 4 n, 0 \leq \varepsilon_{i}<1$, alors il existe une application linéaire continue $E_{\delta, \varepsilon, \lambda}$ de $L_{\delta, \varepsilon}^{2}\left(\mathbb{R}^{n}\right)$ dans $L_{-\delta, \varepsilon}^{2}\left(\mathbb{R}^{n}\right)$ dont la norme ne dépend pas de $\lambda$ telle que pour toute fonction $f$ dans $L_{\hat{\delta}, \varepsilon}^{2}\left(\mathbb{R}^{n}\right)$ on ait les égalités.

$$
P_{\lambda}(\xi, D) E_{\delta, \varepsilon, \lambda} f=f ; \quad E_{\delta, \varepsilon, \lambda} P_{\lambda}(\xi, D) f=f .
$$

On désigne, pour tout $\rho>0$, par $V_{\rho}$ l'ouvert de $\mathbb{R}^{\omega}$ des $|c|>2 \rho$. La preuve de la proposition 2 passe par l'assertion suivante.

Proposition 3. Soit $P(C, D)=\sum_{|\alpha| \leq m} C_{\alpha} D^{\alpha}$ un opérateur différentiel a coefficients constants. On peut trouver:

a) Une suite $\left(\theta_{j, k}\right)$ de points de $\mathbb{R}^{n}$ indexée par les couples $(j, k)$ où $j$ est un entier positif et $k$ un point de à coordonnées entières $\left\|\theta_{j, k}\right\| \leq \delta / 2 \pi$.

b) Une partition $C_{c}^{\infty}\left(\mathbb{R}^{n+\omega}\right)$ de l'unité de $\mathbb{R}^{n+\omega}$, go $g^{j, k}$ qui satisfont les propriétés ci-dessous:

$1^{\circ}$ Pour tout couple $(j, k)$ on note $E_{\rho}^{j, k}$ la distribution sur $\mathbb{R}^{n}$ définie par la formule:

$$
f \rightarrow\left\langle E_{\delta}^{j, k}, f\right\rangle=\int_{\boldsymbol{R}^{n}} g_{\rho}^{j, k}(c, \xi) \int_{|z|=1} \frac{\widehat{f}\left(2 \pi\left(\xi+z \theta_{j, k}\right)\right) d \xi d z}{2 \pi i z P\left(c, 2 \pi\left(\xi+z \theta_{j, k}\right)\right)}
$$

et

$$
\widehat{f}(\xi)=\int_{\mathbb{R}^{n}} e^{-i x \cdot \xi} f(x) d x .
$$

Alors $E_{\delta}=\sum_{k, j} E_{\rho}^{j, k}$ est une distribution qui dépend de manière $C^{\infty}$ des $C$ dans $V_{\rho}$.

$2^{\circ}$ Elle définit par convolution un opérateur borné de $\mathscr{L}_{\delta}$ dans $\mathscr{F}_{-\delta}$ dont la norme ne dépasse pas une constante fois $1 / \rho$, où $\mathscr{F}_{\delta}$, resp. 
$\mathscr{F}_{-\delta}$, désigne $F_{\delta, 0}$, resp. $F_{-\delta, 0}$, telle que $P E_{\delta}=I_{d}$.

Cette proposition est prouvée dans [5]. Nous voulons seulement en déduire un corollaire dont découle la preuve de la proposition 2 .

Lemme 4. Soint $\varphi \in C_{c}^{\infty}\left(\boldsymbol{R}^{n}\right)$ et $\beta$ un $n$ multi-indice. On définit une fonction $\psi$ dans $\Gamma_{J}$ par: $\psi=\left(E_{\delta} * \varphi\right) \circ \log$. Alors $\left|\frac{\partial^{\beta} \psi}{\partial x^{\beta}}\right| \leq C(\varphi, x)$ $\times \prod_{\beta=\left(\beta_{1}, \cdots, \beta_{r}\right)}\left|x_{j}\right|^{-\beta_{j}}$ avec $C(\varphi, x) \simeq e^{\delta_{\|1 \log x\|}}$ quand $\|\log x\| \rightarrow+\infty$.

Preuve du lemme. Par définition, $\psi$ est la somme en $(j, k)$ des termes $F_{j, k}$, que nous allons examiner:

$$
\begin{aligned}
& F_{j, k}(x)=\int_{\boldsymbol{R}^{n}} g_{\rho}^{j, k}(c, \xi) \\
& \quad \times \int_{|z|=1} \frac{\exp \left(2 \pi i(\log x)\left(\xi+z \theta_{j, k}\right)\right) \hat{\varphi}\left(-2 \pi\left(\xi+z \theta_{j, k}\right)\right) d z d \xi}{2 \pi i z P\left(c, 2 \pi\left(\xi+z \theta_{j, k}\right)\right)} .
\end{aligned}
$$

Mais il est facile de vérifier par récurrence que:

$$
\begin{gathered}
\frac{\partial^{\beta} F_{j, k}}{\partial x^{\beta}}(x)=\prod_{\beta=\left(\beta_{1}, \cdots, \beta_{r}\right)} x_{j}^{-\beta_{j}} \int_{\boldsymbol{R}^{n}} \frac{g^{j, k}(c, \xi)}{(1+\|\xi\|)^{n+1}} \int_{|z|=1} \exp \left(-2 \pi i(\log x) \cdot\left(\xi+z \theta_{j, k}\right)\right) \\
\times \frac{(1+\|\xi\|)^{n+1} R_{j, k}\left(\frac{\partial}{\partial x}\right) \varphi\left(-2 \pi\left(\xi+z \theta_{j, k}\right)\right)}{2 \pi i P\left(c, 2 \pi\left(\xi+z \theta_{j, k}\right)\right)} d \xi d z
\end{gathered}
$$

où $R_{j, k}$ est un polynôme dont des coefficients sont bornées en $j$ et $k$.

On vérifie facilement, puisque $\left\|\theta_{j, k}\right\| \leq \delta / 2 \pi$ que si l'on désigne par $\left(R_{j, k}\left(\frac{\partial}{\partial x}\right) \varphi\right)_{X}$ la fonction $\left(R_{j, k}\left(\frac{\partial}{\partial x}\right) \varphi\right)_{X}(t)=\left(R_{j, k}\left(\frac{\partial}{\partial x}\right) \varphi\right)(\log x+t)$ alors:

$$
\begin{aligned}
\mid(1+\|\xi\|)^{n+1} & \widehat{\left(R_{j, k}\left(\frac{\partial}{\partial x}\right) \varphi\right)_{X}}\left(\xi+z \theta_{j, k}\right) \mid \\
& \leq C \int_{\boldsymbol{R}^{n}} e^{\delta\|t\|} \sum_{|s| \leq n+1}\left|D^{s}\left(R_{j, k}\left(\frac{\partial}{\partial x}\right) \varphi\right)(\log x+t)\right| d t \\
& =C \int_{\boldsymbol{R}^{n}} e^{\delta\|\log x-t\|} \sum_{|s| \leq n^{n+1}}\left|D^{s}\left(R_{j, k}\left(\frac{\partial}{\partial x}\right) \varphi\right)(t)\right| d t
\end{aligned}
$$


Puisque $\varphi$ est à support compact on voit immédiatement que cette quantité est équivalente à $e^{\delta\|\log x\|}$ quand $\|\log x\| \rightarrow+\infty$.

Comme $\left|P\left(c, 2 \pi\left(\xi+z \theta_{j, k}\right)\right)\right|^{-1}<c$, voir [5] p. 216, en sommant en $j, k$, on voit immédiatement que:

$$
\left|\frac{\partial^{\beta} \psi}{\partial x^{\beta}}(x)\right| \leq C(x, \varphi) \prod_{\beta=\left(\beta_{1}, \cdots, \beta_{r}\right)}\left|x_{j}\right|^{-\beta_{j}} .
$$

Ce qui termine la preuve du lemme.

Lemme 5. Remarquons que si $x_{i_{1}}, \cdots, x_{i_{r}}$ restent minorer par $\varepsilon_{1}^{0}$ et bornés par $\varepsilon_{2}^{0}$ alors

$$
\left|\frac{\partial^{\beta} \Psi}{\partial x^{\beta}}(x)\right| \leq C\left(\varepsilon_{1}^{0}, \varepsilon_{2}^{0}\right) \prod_{\beta^{\prime}=\left(\beta_{j_{1}}, \cdots, \beta_{j_{s}}\right)}\left|x_{j_{k}}\right|^{-\left(\beta_{j_{k}}+\delta\right)}
$$

où $\beta^{\prime} \subset \beta$ ne contient pas les $\beta_{j}$ pour $\left(j=i_{k}\right)$.

Nous sommes maintenant en mesure de prouver la proposition 2 .

Preuve de la proposition 2. Soient $0<\delta<1 / 4 n$ et $\varepsilon \in \mathbb{R}^{n}, 0 \leq \varepsilon_{i} \leq 1$. Soit $Q_{\lambda}$ l'opérateur différentiel à coefficients constants associé à $P_{\lambda}$ dont l'existence a été prouvée à la proposition 1 . On considère l'opérateur $Q_{\lambda}^{\varepsilon}(D)$ dont le symbole est $Q_{\lambda}^{\varepsilon}(\xi)=Q_{\lambda}(\xi+i \varepsilon / 2)$. On note $\widetilde{E}_{\lambda, 0}^{\delta, \varepsilon}$ une solution fondamentale de l'opérateur $Q_{\lambda}^{\varepsilon}(D)$ comme à la proposition 3 pour la valeur $\delta / 2$ et l'on définit $\widetilde{E}_{\lambda}^{\delta, \varepsilon}$ par la relation:

$$
\varphi \in C_{c}^{\infty}\left(\boldsymbol{R}^{n}\right) \mapsto\left\langle\widetilde{E}_{\lambda}^{\delta, \varepsilon}, \varphi\right\rangle=\left\langle\widetilde{E}_{\lambda, 0}^{\delta, \varepsilon},\left(e^{-\varepsilon \cdot t / 2} \varphi\right)\right\rangle .
$$

Alors:

$$
\widetilde{E}_{\lambda}^{\delta, \varepsilon} * \varphi(x)=e^{-\varepsilon \cdot x / 2}\left(\widetilde{E}_{\lambda, 0}^{\delta, \varepsilon} *\left(e^{+\varepsilon \cdot t / 2} \varphi\right)\right)(x) .
$$

Il s'en suit que $\widetilde{E}_{\lambda}^{\delta, \varepsilon}$ définit par convolution un opérateur borné de $F_{\delta / 2, \varepsilon}$ dans $F_{-\delta / 2, \varepsilon}$ dont la norme ne dépasse pas $M=\operatorname{Inf}_{\alpha, \lambda}\left|q_{\alpha}(\lambda)\right|$ puisque $\widetilde{E}_{\lambda, 0}^{\delta, \varepsilon}$ définit un opérateur borné de $F_{\delta / 2}$ dans $F_{-\delta / 2}$. Pour tout $I \subset J$, toute fonction $U$ de classe $C_{c}^{\infty}\left(\boldsymbol{R}^{n}\right)$ à support disjoint des axes, on définit la fonction $E_{\delta, \varepsilon, \lambda}^{I} U$ par la formule:

$$
E_{\delta, \varepsilon, \lambda}^{I} U(x)=\left(\widetilde{E}_{\lambda}^{\delta, \varepsilon} * \widetilde{U}_{I}\right) \circ \psi_{I}^{-1}(x) .
$$

On pose: 


$$
E_{\delta, \varepsilon, \lambda} U(x)=\sum_{I \subset J} E_{\delta, \varepsilon, \lambda}^{I} U(x) .
$$

D’après ce qui a été dit précédemment, un changement de variables montre tout de suite que $E_{\delta, \varepsilon, \lambda}$ est une application linéaire bornée de $L_{\delta / 2, \varepsilon}^{2}\left(\boldsymbol{R}^{n}\right)$ dans $L_{-\delta / 2, \varepsilon}^{2}\left(\boldsymbol{R}^{n}\right)$ dont la norme ne dépasse pas une constante fois $M$.

Il reste à vérifier la relation algébrique $P_{\lambda}(\xi, D) E_{\delta, \varepsilon, \lambda} U=U$ au sens de distributions. On fera cette vérification pour $n=2$, le cas $n \neq 2$ se traite de la même façon, mais exige plus de notations.

Soit $g \in C_{c}^{\infty}\left(\boldsymbol{R}^{2}\right)$, on veut prouver que $\left\langle P_{\lambda}(\xi, D) E_{\delta, \varepsilon, \lambda} U, g\right\rangle=\langle U, g\rangle$. Il suffit (c'est la méthode générale) d'examiner la cas où $P_{\lambda}=$ $(-i \lambda)^{k_{1}+k_{2}} \xi_{1}^{k_{1}} \xi_{2}^{k_{2}} \frac{\partial^{k_{1}+k_{2}}}{\partial \xi_{1}^{k_{1}} \partial \xi_{2}^{k_{2}}}$ où $k_{1}$ et $k_{2}$ sont des entiers $k_{1}, k_{2} \geq 0$. On ne s'occupera pas de $(-i \lambda)^{k_{1}+k_{2}}$. On veut donc calculer l'expression

$$
\begin{aligned}
& \left\langle P_{\lambda}(\xi, D) E_{\delta, \varepsilon, \lambda} U, g\right\rangle \\
& \quad=\left\langle E_{\delta, \varepsilon, \lambda} U,{ }^{t} P g\right\rangle=(-1)^{k_{1}+k_{2}} \int_{R^{2}} E_{\delta, \varepsilon, \lambda} U(\xi) \frac{\partial^{k_{1}+k_{2}}}{\partial \xi_{1}^{k_{1}} \partial \xi_{2}^{k_{2}}}\left(\xi_{1}^{k_{1}} \xi_{2}^{k_{2}} g\right)(\xi) d \xi \\
& \quad=(-1)^{k_{1}+k_{2}} \sum_{I \subset\{1,2\}} \int_{\Gamma_{I}} E_{\delta, \varepsilon, \lambda}^{I} U(\xi) \frac{\partial^{k_{1}+k_{2}}}{\partial \xi_{1}^{k_{1}} \partial \xi_{2}^{k_{2}}}\left(\xi_{1}^{k_{1}} \xi_{2}^{k_{2}} g\right)(\xi) d \xi .
\end{aligned}
$$

Il suffira encore d'étudier le cas où $I=(1,2)$ les autres se traitant de façon analogue:

$$
\begin{aligned}
A_{1} & =\int_{0}^{+\infty} \int_{0}^{+\infty} E_{\delta, \varepsilon, \lambda}^{I} U(\xi) \frac{\partial^{k_{1}+k_{2}}}{\partial \xi_{1}^{k_{1}} \partial \xi_{2}^{k_{2}}}\left(\xi_{1}^{k_{1}} \xi_{2}^{k_{2}} g\right)(\xi) d \xi \\
& =\lim _{\varepsilon_{1}^{0} \rightarrow 0} \lim _{\varepsilon_{2}^{0} \rightarrow 0} \int_{\varepsilon_{1}^{0}}^{+\infty} \int_{\varepsilon_{2}^{0}}^{+\infty}\left(\widetilde{E}_{\lambda}^{\delta, \varepsilon} * \widetilde{U}_{I}\right) \circ \log (\xi) \frac{\partial^{k_{1}+k_{2}}}{\partial \xi_{1}^{k_{1}} \partial \xi_{2}^{k_{2}}}\left(\xi_{1}^{k_{1}} \xi_{2}^{k_{2}} g\right)(\xi) d \xi .
\end{aligned}
$$

Car l'intégrale $A_{1}$ converge absolument d'après le lemme 4, puisque $\left(\varepsilon_{i}+\delta\right) / 2<1$, d'après les choix de $\varepsilon$ et $\delta$. Mais alors:

$$
\begin{aligned}
\int_{\varepsilon_{1}^{0}}^{+\infty} \int_{\varepsilon_{2}^{0}}^{+\infty}\left(\widetilde{E}_{\lambda}^{\delta, \varepsilon} * \widetilde{U}_{I}\right) \circ \log (\xi) \frac{\partial^{k_{2}+k_{1}}}{\partial \xi_{2}^{k_{2}} \partial \xi_{1}^{k_{1}}}\left(\xi_{2}^{k_{2}} \xi_{1}^{k_{1}} g\right)(\xi) d \xi \\
=\int_{\varepsilon_{1}^{0}}^{+\infty} \sum_{k_{2}^{\prime}=0}^{k_{2}-1}(-1)^{k_{2}^{\prime}} \frac{\partial^{k_{2}^{\prime}}\left(\widetilde{E}_{\lambda}^{\delta, \varepsilon} * \widetilde{U}_{I} \circ \log \left(\xi_{1}, \varepsilon_{2}^{0}\right)\right.}{\partial \xi_{2}^{k_{2}^{\prime}}} \\
\quad \times\left[\frac{\partial^{k_{2}-k_{2}^{\prime}+k_{1}-1}}{\partial \xi_{2}^{k_{2}-k_{2}^{\prime}-1} \partial \xi_{1}^{k_{1}}}\left(\xi_{1}^{k_{1}} \xi_{2}^{k_{2}} g\right)\left(\xi_{1}, \xi_{2}\right)\right]_{\xi_{2}=\varepsilon_{2}^{0}} d \xi_{1} \\
\quad+(-1)^{k_{2}} \int_{\varepsilon_{1}^{0}}^{+\infty} \int_{\varepsilon_{2}^{0}}^{+\infty} \xi_{2}^{k_{2}} \frac{\partial^{k_{2}}}{\partial \xi_{2}^{k_{2}}}\left(\left(\widetilde{E}_{\lambda}^{\delta, \varepsilon^{\prime}} * \widetilde{U}_{I}\right) \circ \log \left(\xi_{1}, \xi_{2}\right)\right)
\end{aligned}
$$




$$
\times \frac{\partial^{k_{1}}\left(\xi_{1}^{k_{1}} g\right)\left(\xi_{1}, \xi_{2}\right) d \xi_{1} d \xi_{2}}{\partial \xi_{1}^{k_{1}}}
$$

Puisque $g$ est à support compact, il est facile de vérifier que:

$$
\left|\frac{\partial^{k_{2}-k_{2}^{\prime}-1}}{\partial \xi_{2}^{k_{2}-k_{2}-1}}\left[\xi_{2}^{k_{2}} \frac{\partial^{k_{1}}}{\partial \xi_{1}^{k_{1}}}\left(\xi_{1}^{k_{1}} g\right)\left(\xi_{1}, \xi_{2}\right)\right]\right| \leq C\left|\xi_{2}\right|^{k_{2}^{\prime}+1}
$$

D'autre part puisque $\widetilde{U}_{I}$ est $C_{c}^{\infty}\left(\boldsymbol{R}^{n}\right)$, d'après le lemme 5

$$
\left|\frac{\partial^{k_{2}^{\prime}}}{\partial \xi_{2}^{k_{2}^{\prime}}}\left[\left(\widetilde{E}_{\lambda}^{\delta, \varepsilon} * \widetilde{U}_{I}\right) \circ \log (\xi)\right]\right| \leq c\left(\widetilde{U}_{I}\right)\left|\xi_{2}\right|^{-k_{2}^{\prime}-\left(\delta+\varepsilon_{2}^{0}\right) / 2}\left|\xi_{1}\right|^{-\left(\delta+\varepsilon_{1}^{0}\right) / 2}
$$

et:

$$
\begin{aligned}
& {\left[\mid \frac{\partial^{k_{2}^{\prime}}}{\partial \xi_{2}^{k_{2}^{\prime}}}\left(\widetilde{E}_{\lambda}^{\delta, \varepsilon} * \widetilde{U}_{I} \circ \log \left(\xi_{1}, \xi_{2}\right) \frac{\partial^{k_{2}-k_{2}^{\prime}+k_{1}-1}}{\partial \xi_{2}^{k_{2}-k_{2}^{\prime}+k_{1}-1}}\left(\xi_{1}^{k_{1}} \xi_{2}^{k_{2}} g\right)\left(\xi_{1}, \xi_{2}\right) \mid\right]_{\xi_{2}=\varepsilon_{2}^{0}}\right.} \\
& \quad \leq\left(\varepsilon_{2}^{0}\right)^{1-\left(\delta+\varepsilon_{2}^{0}\right) / 2}\left|\xi_{1}\right|^{-\left(\delta+\varepsilon_{1}^{0}\right) / 2}\left|g_{1}(\xi)\right|
\end{aligned}
$$

où $g_{1}$ est une fonction à support compact dont l'existence est assurée par le fait que $g$ est à support compact. Le théorème de convergence dominée de Lebesgue prouve alors que:

$$
\begin{gathered}
\lim _{\varepsilon_{2}^{0} \rightarrow 0} \int_{\varepsilon_{1}^{0}}^{+\infty} \sum_{k_{2}^{\prime}=0}^{k_{2}-1}(-1)^{k_{2}^{\prime}} \frac{\partial^{k_{2}^{\prime}}\left(\widetilde{E}_{\lambda}^{\delta, \varepsilon} * \widetilde{U}_{I}\right) \circ \log \left(\xi_{1}, \varepsilon_{2}^{0}\right)}{\partial \xi_{2}^{k_{2}^{\prime}}} \\
\times \frac{\partial^{k_{2}-k_{2}^{\prime}+k_{1}-1}\left(\xi_{1}^{k_{1}} \xi_{2}^{k_{2}} g\right)\left(\xi_{1}, \varepsilon_{2}^{0}\right) d \xi_{1}}{\partial \xi_{2}{ }^{k_{2}-k_{2}^{\prime}-1} \partial \xi_{1}^{k_{1}}}=0
\end{gathered}
$$

et :

$$
\begin{gathered}
A_{1}=\lim _{\varepsilon_{1}^{0} \rightarrow 0} \lim _{\varepsilon_{2}^{0} \rightarrow 0} \int_{\varepsilon_{1}^{0}}^{+\infty} \int_{\varepsilon_{2}^{0}}^{+\infty}(-1)^{k_{2}} \xi_{2}^{k_{2}} \frac{\partial^{k_{2}}}{\partial \xi_{2}^{k_{2}}}\left(\widetilde{E}_{\lambda}^{\delta, \varepsilon} * \widetilde{U}_{I}\right) \circ \log \left(\xi_{1}, \xi_{2}\right) \frac{\partial^{k_{1}}}{\partial \xi_{1}^{k_{1}}} \\
\times\left(\xi_{1}^{k_{1}} g\right)\left(\xi_{1}, \xi_{2}\right) d \xi_{1} d \xi_{2} .
\end{gathered}
$$

On utilise encore le lemme 5 pour voir que l'intégrale

$$
A_{1}^{\prime}=\int_{0}^{+\infty} \int_{0}^{+\infty} \xi_{2}^{k_{2}} \frac{\partial^{k_{2}}}{\partial \xi_{2}^{k_{2}}}\left(\widetilde{E}_{\lambda}^{\delta, \varepsilon} * \widetilde{U}_{I}\right) \circ \log \left(\xi_{1}, \xi_{2}\right) \frac{\partial^{k_{1}}}{\partial \xi_{1}^{k_{1}}}\left(\xi_{1}^{k_{1}} g\right)\left(\xi_{1}, \xi_{2}\right) d \xi_{1} d \xi_{2} .
$$

Converge absolument par conséquent on a l'égalité $A_{1}^{\prime}=A_{1}$ et:

$$
\begin{gathered}
A_{1}^{\prime}=\lim _{\substack{0 \\
\varepsilon_{2} \rightarrow 0}} \lim _{\varepsilon_{1}^{0} \rightarrow 0}(-1)^{k_{2}} \int_{\varepsilon_{2}^{0}}^{+\infty} \int_{\varepsilon_{1}^{0}}^{+\infty} \xi^{k_{2}} \frac{\partial^{k_{2}}}{\partial \xi_{2}^{k_{2}}}\left(\widetilde{E}_{\lambda}^{\delta, \varepsilon} * \widetilde{U}_{I}\right) \circ \log \left(\xi_{1}, \xi_{2}\right) \\
\times \frac{\partial^{k_{1}}}{\partial \xi_{1}^{k_{1}}}\left(\xi_{1}^{k_{1}} g\right)\left(\xi_{1}, \xi_{2}\right) d \xi_{1} d \xi_{2} .
\end{gathered}
$$


On refait le même raisonnement et l'on voit que:

$$
A_{1}=(-1)^{k_{2}+k_{1}} \lim _{\varepsilon_{2}^{0} \rightarrow 0} \lim _{\varepsilon_{1}^{0} \rightarrow 0} \int_{\varepsilon_{2}^{0}}^{+\infty} \int_{\varepsilon_{1}^{0}}^{+\infty} \xi_{1}^{k_{1}} \xi_{2}^{k_{2}} \frac{\partial^{k_{1}+k_{2}}\left(\widetilde{E}_{\lambda}^{\delta, \varepsilon} * \widetilde{U}_{I}\right) \circ \log (\xi) g(\xi) d \xi}{\partial \xi_{1}^{k_{1}} \partial \xi_{2}^{k_{2}}}
$$

Comme $E_{\lambda, 0}^{\delta, \varepsilon}$ est une solution fondamentale, on voit tout de suite que:

$$
\begin{aligned}
A_{1} & =(-1)^{k_{2}+k_{1}} \lim _{\varepsilon_{2}^{0} \rightarrow 0} \lim _{\varepsilon_{1}^{0} \rightarrow 0} \int_{\varepsilon_{2}^{0}}^{+\infty} \int_{\varepsilon_{1}^{0}}^{+\infty} U(\xi) g(\xi) d \xi \\
& =\int_{0}^{+\infty} \int_{0}^{+\infty} U(\xi) g(\xi) d \xi
\end{aligned}
$$

si l'on fait la sommation sur $I$, on voit que l'on a l'égalité $\left\langle P_{\lambda} E_{\delta, \varepsilon, \lambda} U, g\right\rangle$ $=\langle U, g\rangle$ voulue.

Remarquons que $L_{-\delta / 2, \varepsilon}^{2}$ se plonge continûment dans $\mathscr{D}^{\prime}\left(\boldsymbol{R}^{n}\right)$ et la formule $\left\langle P_{\lambda} E_{\delta, \varepsilon, \lambda} U, g\right\rangle$ a toujours un sens puisque $E_{\delta, \varepsilon, \lambda} U$ est dans $L_{\delta / 2, \varepsilon}^{2}\left(\boldsymbol{R}^{n}\right)$ d'après la proposition 3 .

On remarque aussi que le sous-espace de $L_{\delta / 2, \varepsilon}^{2}\left(\boldsymbol{R}^{n}\right)$ constitué des fonctions à support disjoint des axes est dense dans $L_{\delta / 2, \varepsilon}^{2}\left(\boldsymbol{R}^{n}\right)$.

En effet si cela n'était pas vrai, il existerait une fonction $f \in L_{\delta / 2, \varepsilon}^{2}\left(\boldsymbol{R}^{n}\right)$ qui ne soit pas dans l'adhérence de ce sous-space. D'où une forme linéaire (d'après Hahn-Banach), par conséquent une fonction $F$ de $L_{\delta / 2, \varepsilon}^{2}\left(\boldsymbol{R}^{n}\right)$ qui soit triviale sur ce sous-espace telle que

$$
\int_{\boldsymbol{R}^{n}} f(x) \bar{F}(x)\left|X_{J}\right|^{\varepsilon-1} e^{-\delta / 2\|\log x\|} d x=1 .
$$

Mais il est clair qu'unetelle fonction $F$ s'annule en de hors des axes par conséquent il y a une contradiction.

Puisque nous venons de prouver l'égalité $\left\langle P_{\lambda} E_{\delta, \varepsilon, \lambda} U, h\right\rangle=\langle U, h\rangle$ pour les fonctions $U \in C_{c}^{\infty}\left(\mathbb{R}^{n}\right)$ à support disjoint des axes, les deux remarques précèdentes prouvent que cette égalité subsiste quand $U \in L_{\delta / 2, \varepsilon}^{2}\left(\boldsymbol{R}^{n}\right)$. Ce qui termine la preuve de la première égalité; la seconde se prouve de la même façon.

On se propose d'énoncer la dernière proposition dont on aura besoin pour établir le théorème $I^{\prime}$. Pour formuler cette proposition on aura besoin de quelques notations.

Notations. Soit $P_{t}$ le noyau de Poisson de $\theta_{\Gamma_{J}}=\left\{(y, t) \in \boldsymbol{R}^{(n+1)}\right.$, 
$\left.t_{i}>0\right\}: P_{t, t_{n+1}}(y, z)=\prod_{j \in J} \frac{t_{j}}{t_{j}^{2}+y_{j}^{2}} \frac{t_{n+1}}{t_{n+1}^{2}+z^{2}}$ si $f$ est une distribution raisonnable sur $H_{n}$, on définit un prolongement de $f$ par:

$$
F\left(x, y+i t, z+i t_{n+1}\right)=\int_{\mathbb{R}^{n+1}} P_{t, t_{n+1}}((y, z)-(v, u)) f(x, v, u) d v d u .
$$

Par suite d'énormes notations qui s'introduisent quand on vent écrire les énoncés pour $n$ quelconque, nous supposons $n=2$ dans la définition suivante.

Soit $f$ une distribution raisonnable sur $H_{3}$ et soit $p=\left(p_{1}, p_{2}, p_{3}\right), 0 \leq p_{i}$ $\leq 1$ on définit $\|f\|_{\vec{p}}^{2}$ comme étant égale à la quantité suivante, finie ou infinie:

$$
\begin{aligned}
\|f\|_{\vec{p}}^{2}=\int_{\boldsymbol{R}^{2}} & \left\{\int _ { \boldsymbol { R } } \left\{\int _ { \boldsymbol { R } } \left\{\int_{\Gamma_{J}}\left[\left|\frac{\partial^{3} F\left(x, y+i t, z+i t_{3}\right)}{\partial t_{1} \partial t_{2} \partial t_{3}}\right|^{2} t_{1} t_{2} t_{3} d t_{1} d t_{2} d t_{3}\right]^{p_{1} / 2}\right.\right.\right. \\
& \left.\left.\left.\times d y_{1}\right\}^{p_{2} / p_{1}} d y_{2}\right\}^{p_{3} / p_{2}} d z\right\}^{2 / p_{3}} d z
\end{aligned}
$$

On note $R_{\vec{p}}$ l'espace des $f$ telles que $\|f\|_{\vec{p}}<\infty$.

Maintenant si $\vec{p}=\left(p_{1}, \cdots, p_{n+1}\right), 1 \leq p_{i}$, on pose:

$$
\begin{aligned}
\|f\|_{2, \vec{p}}^{2}=\int_{\boldsymbol{R}^{n}} & {\left[\int_{-\infty}^{+\infty} \cdots\left(\int_{-\infty}^{+\infty}\left|f\left(x, y_{1}, y_{2}, \cdots, y_{n}, z\right)\right|^{p_{1}} d y_{1}\right)^{p_{2} / p_{1}}\right.} \\
& \left.\times d y_{2} \cdots d z\right]^{2 / p_{n+1}} d x
\end{aligned}
$$

On note $L_{2, \vec{p}}\left(H_{n}\right)$ l'espace des $f$ telles que $\|f\|_{2, \vec{p}}<\infty$.

Soient $\vec{q} \in \mathbb{R}^{n}, \vec{q}_{i} \geq 1$, on pose $\vec{q}^{0}=(\vec{q}, 16 / 13) ; \vec{q}^{1}=(\vec{q}, 16 / 9) ;$ on va prouver la proposition suivante:

Proposition 6. Soient $\delta$, $\varepsilon$ comme précédemment. Il existe une constante $c_{\delta, \varepsilon}$ telle que:

$1^{\circ} \quad$ a) $\quad\left\|F_{\delta, \varepsilon}(g)\right\|_{L^{2}\left(H_{n}\right)}^{2} \leq c_{\delta, \varepsilon} \sum_{|s| \leq n+2}\left\|D^{s} g\right\|_{L^{2}\left(H_{n}\right)}^{2}$

$$
\left\|H_{\delta, \varepsilon}(g)\right\|_{L^{2}\left(H_{n}\right)}^{2} \leq c_{\delta, \varepsilon} \sum_{|s| \leq n+2}\left\|D^{s} g\right\|_{L^{2}\left(H_{n}\right)}^{2}
$$

b) Soit $p$ suffisamment proche de un pour que $0<\left(1-\varepsilon_{j}+\delta\right) p<1$ alors:

$$
\left\|F_{\delta, \varepsilon}^{\prime}(g)\right\|_{L^{2\left(H_{n}\right)}}
$$




$$
\begin{aligned}
& \quad \leq c_{\delta, \varepsilon}\left(\sum_{|s| \leq 3 n+1}\left\|(1+\|x\|)^{3 n} D^{s} g\right\|_{2}^{2}\right)^{1 / 2 p^{\prime}}\left\|(1+\|(x, y, z)\|)^{3 n} g\right\|_{L^{2}\left(\bar{H}_{n}\right)}^{\left(p^{\prime}-2\right) / p^{\prime}} \\
& \left\|H_{\delta, \varepsilon}^{\prime}(g)\right\|_{L^{2}\left(H_{n}\right)} \\
& \quad \leq c_{\delta, \varepsilon}\left(\sum_{|s| \leq 3 n+2}\left\|(1+\|x\|)^{3 n} D^{s} g\right\|_{2}^{2}\right)^{1 / 2 p^{\prime}}\left\|(1+\|(x, y, z)\|)^{3 n} g\right\|_{L^{1}\left(\bar{H}_{n}\right)}^{\left(p^{\prime}-2\right) / p^{\prime}}
\end{aligned}
$$

$2^{\circ}$ On pose:

a) $\forall j \in I \subset J, 1 / P_{I, j}=1-\left(\delta-\varepsilon_{j}\right) / 2 ; \forall k \notin I, 1 / P_{I, k}=1-\left(\varepsilon_{k}+\delta\right) / 2$. Alors:

$$
\left\|H_{\delta, \varepsilon}^{\prime}(g)\right\|_{L^{2}\left(H_{n}\right)} \leq c_{\delta, \varepsilon} \sum_{I \subset J}\|g\|_{2, \vec{p}_{T}^{1}}+\|g\|_{2, \vec{p}_{T}^{0}} .
$$

b) On suppose $\varepsilon=0$, on pose $1 / p_{j}=1+\delta / 2, j \in I$ et $1 / p_{k}=1-\delta / 2$. Alors:

$$
\left\|H_{\delta, \varepsilon}(g)\right\|_{L^{2\left(H_{n}\right)}} \leq c_{\delta, \varepsilon}\left(\sum_{I \subset J}\|g\|_{\vec{P}_{I}^{0}}+\|g\|_{\vec{P}_{I}^{1}}\right) .
$$

Preuve de la proposition. $1^{\circ}$ L'estimation de $F_{\delta, \varepsilon}$ est analogue à celle de $H_{\delta, \varepsilon}$; celle de $F_{\delta, \varepsilon}^{\prime}$ est aussi la même que $H_{\delta, \varepsilon}^{\prime}$. On se contentera donc des estimations de $H_{\delta, \varepsilon}$ et $H_{\delta, \varepsilon}^{\prime}$.

a) On va utiliser la transformation de Fourier. Pour tout $\lambda \in \mathbb{R}$, le noyau de la transformee de Fourier de $H_{\delta, \varepsilon}(g)$ vaut:

$$
\widehat{T}(x, \xi, \lambda)=\widehat{g}_{2,3}^{0}(x-\xi, \lambda \xi, \lambda) \sum_{I \subset J} \varphi_{I}^{0}(\xi) \varphi_{I c}^{1}(\xi)\left|\xi_{I}\right|^{(1-\varepsilon+\delta) / 2}\left|\xi_{I c}\right|^{(1-\varepsilon-\delta) / 2}|\lambda|^{\delta_{0}}
$$

où

$$
\widehat{g}_{2,3}^{0}(x, \lambda \xi, \lambda)=\int_{\boldsymbol{R}^{n+1}} g(x, y, z) e^{i \lambda(y \cdot \xi-z)} d y d z
$$

Mais

$$
\operatorname{Tr}\left[\widehat{T}(\lambda) \circ \widehat{T}^{*}(\lambda)\right] \leq \int_{\boldsymbol{R}^{2 n+1}}|\widehat{T}(x, \xi, \lambda)|^{2} d x d \xi
$$

Par conséquent

$$
\begin{aligned}
& \int_{-\infty}^{+\infty} \operatorname{Tr}\left[\widehat{T}(\lambda) \circ \widehat{T}^{*}(\lambda)\right]|\lambda|^{n} d \lambda \\
& \leq \int_{\boldsymbol{R}^{2 n+1}} \sum_{I \subset J}\left|\lambda \xi_{I}\right|^{1-\varepsilon+\delta}\left|\lambda \xi_{I c^{\prime}}\right|^{1-\varepsilon-\delta}\left|\widehat{g}_{2,3}^{0}(x, \lambda \xi, \lambda)\right|^{2}|\lambda|^{2 \delta_{0}+\varepsilon \cdot 1-\delta\left(\left|I_{1}-\right| I c^{\prime} \mid\right)}|\lambda|^{n} \\
& \times d \lambda d \xi d x
\end{aligned}
$$


(d'après le choix de $\delta$ )

$$
\begin{aligned}
\leq c_{2, \delta} \int_{\boldsymbol{R}^{2 n+1}} & (1+\|\lambda \xi\|)^{n\left(1-\varepsilon_{0}^{\prime}+\delta\right)}\left|\widehat{g}_{2,3}^{0}(x, \lambda \xi, \lambda)\right|^{2}|\lambda|^{n}(1+|\lambda|) d \lambda d \xi d x \\
\varepsilon_{0}^{\prime}=\min \varepsilon_{i} ; & =c_{\varepsilon, \delta} \int_{\boldsymbol{R}^{2 n+1}}(1+\|\xi\|)^{n\left(1-\varepsilon_{0}^{\prime}+\delta\right)}(1+|\lambda|)\left|\widehat{g}_{2,3}^{0}(x, \xi, \lambda)\right|^{2} d \lambda d \xi d x \\
& \leq c_{\varepsilon, \delta} \sum_{\substack{|s| \leq n\left(1-\varepsilon_{0}^{\prime}+\delta\right) \\
|p| \leq 1}}\left\|D_{y}^{s} D_{z}^{p} g\right\|_{L^{2}\left(H_{n}\right)}^{2} \\
& \leq c_{\varepsilon, \delta} \sum_{|s| \leq n+3 / 2}\left\|D^{s} g\right\|_{L^{2}\left(H_{n}\right)}^{2}
\end{aligned}
$$

ce qu'il fallait prouver pour $H_{\delta, \varepsilon}$.

b) Ouvrons une parenthèse pour $H_{\delta, \varepsilon}^{\prime}$.

On reprend toutes les estimations précédentes jusqu'à *; il suffit d'examiner le terme:

$$
\begin{aligned}
& \left.\int_{\boldsymbol{R}^{2 n+1}} \varphi_{I}^{0}(\xi) \varphi_{I^{c}}^{1}(\xi) !(\lambda \xi)_{I}\right|^{-(1-\varepsilon+\delta)}\left|\lambda \xi_{I^{c}}\right|^{-(1-\varepsilon-\delta)} \\
& \times\left|\widehat{g}_{2,3}^{0}(x, \lambda \xi, \lambda)\right|^{2}|\lambda|^{n}|\lambda|^{-2 \delta_{0}+(n-\varepsilon \cdot 1)+\delta\left(|I|-\left|I^{c}\right|\right)} d \xi d \lambda d x \\
& \leq \int_{\substack{\left|\xi_{i}\right| \leq|\lambda|, i \in I \\
\xi_{j}|\geq \lambda|, j \in I}}\left|\xi_{I}\right|^{-(1-\varepsilon+\delta)}\left|\xi_{I c}\right|^{-(1-\varepsilon-\delta)} \\
& \times\left|\widehat{g}_{2,3}^{0}(x, \xi, \lambda)\right|^{2}|\lambda|^{-2 \delta_{0}+(n-\varepsilon \cdot 1)+\delta\left(|I|-\mid I c^{c \mid}\right)} d \xi d \lambda d x
\end{aligned}
$$

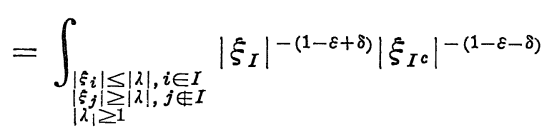

$$
\begin{aligned}
& \times\left|\widehat{g}_{2,3}^{0}(x, \xi, \lambda)\right|^{2}|\lambda|^{-2 \delta_{0}+(n-\varepsilon \cdot 1)+\delta\left(|I|-\left|I^{c}\right|\right)} d \xi d \lambda d x
\end{aligned}
$$

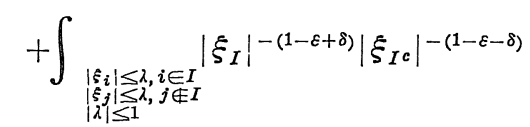

$$
\begin{aligned}
& \times\left|\widehat{g}_{2,3}^{0}(x, \xi, \lambda)\right|^{2}|\lambda|^{-2 \delta_{0}+(n-\varepsilon \cdot 1)+\delta\left(|I|-\left|I^{c}\right|\right)} d \xi d \lambda d x .
\end{aligned}
$$

Puisque $-5 / 8 \leq-2 \delta_{0}+(n-\varepsilon \cdot 1)+\delta\left(|I|-\left|I^{c}\right|\right) \leq-1 / 8$, ces expressions ne dépassent pas:

$$
\begin{aligned}
& \int_{\boldsymbol{R}^{n+1}}\left|\xi_{I}\right|^{-(1-\varepsilon+\delta)}\left|\xi_{I^{c}}\right|^{-(1-\varepsilon-\delta)}\left|\widehat{g}_{2,3}^{0}(x, \xi, \lambda)\right|^{2}|\lambda|^{-5 / 8} d \xi d \lambda d x \\
& \quad+\int_{\boldsymbol{R}^{n+1}}\left|\xi_{I}\right|^{-(1-\varepsilon+\delta)}\left|\widehat{g}_{2,3}^{0}(x, \xi, \lambda)\right|^{2} d \xi d \lambda d x \\
& =A_{1}+A_{2} .
\end{aligned}
$$


Soit $1<p$ assez voisin de 1 . Alors d'après le choix de $\delta$ et $\varepsilon$

$$
\prod_{i=1}^{n}\left(1+\xi_{1}^{2}\right)^{-p}\left|\xi_{I}\right|^{-p(1-\varepsilon-\delta)}\left|\xi_{I^{c}}\right|^{-p(1-\varepsilon-\delta)}\left(1+\lambda^{2}\right)^{-p}|\lambda|^{-5 p / 8} \prod_{i=1}^{n}\left(1+x_{i}^{2}\right)^{-p}
$$

et: $h_{2}^{p}(x, \xi, \lambda)=\prod_{i=1}^{n}\left(1+\xi_{i}^{2}\right)^{-p}\left(1+x_{i}^{2}\right)^{-p}\left(1+\lambda^{2}\right)^{-p}$ sont intégrables.

$$
\begin{aligned}
A_{1} \leq & \left(\int_{\boldsymbol{R}^{n+1}} h_{1}^{p}(\xi) d \xi\right)^{1 / p}\left[\int_{\boldsymbol{R}^{2 n+1}}\left|\widehat{g}_{2,3}^{0}(x, \xi, \lambda)\right|^{2}\left|h_{1}^{-1}(x, \xi, \lambda)\right|^{p^{\prime}} d x d \xi d \lambda\right]^{1 / p^{\prime}} \\
\leq & c_{\delta, \varepsilon}\left(\sum_{|s| \leq 3 n+2}\left\|(1+\|x\|)^{3 n} D^{s} g\right\|_{2}^{2}\right)^{1 / p^{\prime}} \\
& \times\left(\sum_{|s| \leq 3 n+2}\left\|(1+\|(x, y, z)\|)^{3 n} g\right\|_{L^{1\left(H_{n}\right)}}\right)^{\left(p^{\prime}-2\right) / p^{\prime}}
\end{aligned}
$$

où $1 / p+1 / p^{\prime}=1$, d'après l'inégalité de convexité de Riesz.

L'estimation de $A_{2}$ est analogue avec $h_{2}$; l'estimation suivante n'est qu'une amélioration de celle-ci.

$2^{\circ}$ a) On part encore de $\circledast$ et l'on veut faire des estimations plus fines de cette intégrale. On peut trouver une constante $\Gamma(I, \varepsilon, \delta)$ telle que l'intégrale à estimer soit le carré de la norme $L^{2}\left(H_{n}\right)$ de la fonction:

$$
\begin{aligned}
& G_{I, \delta, \varepsilon}(x, y, z) \\
& \quad=\Gamma(I, \varepsilon, \delta) \int_{\boldsymbol{R}^{n+1}} \frac{g(x, y+t, z+s)|s|^{-1+\left(\delta_{0}-(n-\varepsilon \cdot 1) / 2\right)-\left(\delta\left(|I|-\left|I^{c}\right|\right) / 2\right)} d s d t}{\left|t_{I}\right|^{(1+\varepsilon-\delta) / 2}\left|t_{I c}\right|^{(1+\varepsilon+\delta) / 2}} .
\end{aligned}
$$

A cause de la longueur des notations, on va supposer que $n=2$; on laisse de côté la constante multiplicative qui intervient dans la définition de $G_{I, \varepsilon, \delta}$. On prend par exemple $I=\{1\}$, ce qui ne restreint nullement la généralité de la méthode.

$$
\begin{aligned}
& \int_{\boldsymbol{R}^{3}}\left|G_{I, \delta, \varepsilon}(x, y, z)\right|^{2} d y d z \\
& \leq \int_{\boldsymbol{R}^{2}} \int_{-\infty}^{+\infty}\left|\int_{-\infty}^{+\infty} \int_{\boldsymbol{R}^{2}} \frac{|g(x, y+t, z+s)| d t d s}{\left|t_{1}\right|^{(1+\varepsilon-\delta) / 2}\left|t_{2}\right|^{(1+\varepsilon+\delta) / 2}|s|^{11 / 16}}\right|^{2} d y d z \\
& \quad+\int_{\boldsymbol{R}^{2}} \int_{-\infty}^{+\infty}\left|\int_{-\infty}^{+\infty} \int_{\boldsymbol{R}^{2}} \frac{|g(x, y+t, z+s)| d s d t}{\left|t_{1}\right|^{(1+\varepsilon-\delta) / 2}\left|t_{2}\right|^{(1+\varepsilon+\delta) / 2}|s|^{15 / 16}}\right|^{2} d y d z \\
& =\int_{\boldsymbol{R}^{3}}\left|A_{3}(x, y, z)\right|^{2} d x d y d z+\int_{\boldsymbol{R}^{3}}\left|A_{4}(x, y, z)\right|^{2} d x d y d z
\end{aligned}
$$

Les deux fonctions $A_{3}$ et $A_{4}$ se traitent de la même façon; examinons $A_{3}(x)$ par exemple: en posant $p_{3}=16 / 13$ et $p_{4}=16 / 9$ 


$$
\begin{aligned}
& \int_{\boldsymbol{R}^{3}}\left[A_{3}(x, y, z)\right]^{2} d y d z \\
& \quad \leq c_{\delta, \varepsilon}^{\prime} \int_{\mathbb{R}^{2}}\left(\int_{\boldsymbol{R}}\left|\int_{\boldsymbol{R}^{2}} \frac{|g(x, y+t, z)| d t_{1} d t_{2}}{\left|t_{1}\right|^{(1+\varepsilon-\delta) / 2}\left|t_{2}\right|^{(1+\varepsilon+\delta) / 2}}\right|^{p_{3}} d z\right)^{2 / p_{3}} d y
\end{aligned}
$$

d'après [2].

On utilise l'inégalité de Minkowski pour trouver que cette intégrale ne dépasse pas:

$$
\left(\int_{\mathbb{R}^{2}}\left(\int_{\boldsymbol{R}^{2}}\left|\int_{\boldsymbol{R}^{2}} \frac{|g(x, y+t, z)| d t_{1} d t_{2}}{\left|t_{1}\right|^{(1+\varepsilon-\delta) / 2}\left|t_{2}\right|^{(1+\varepsilon+\delta) / 2}}\right|^{2} d y\right)^{p_{3} / 2}\right)^{2 / p_{3}}
$$

on recommence le même argument pour voir que:

$$
\begin{aligned}
& \int_{\boldsymbol{R}^{2}}\left|\int_{\boldsymbol{R}^{2}} \frac{g(x, y+t, z) d t_{1} d t_{2}}{\left|t_{1}\right|^{\left(1+\varepsilon_{1}-\delta\right) 2}\left|t_{2}\right|^{\left(1+\varepsilon_{2}+\delta\right) / 2}}\right|^{2} d y \\
& \quad \leq c_{\delta, \varepsilon}^{\prime \prime}\left(\int_{-\infty}^{+\infty}\left(\int_{-\infty}^{+\infty}\left|g\left(x, y_{1}, y_{2}, z\right)\right|^{p_{1}} d y_{1}\right)^{p_{2} / p_{1}} d y_{2}\right)^{2 / p_{2}} .
\end{aligned}
$$

Par conséquent:

$$
\begin{aligned}
& \int_{\boldsymbol{R}^{3}}\left[A_{3}(x, y, z)\right]^{2} d y d z \\
& \leq c_{\delta, \varepsilon}\left(\int_{-\infty}^{+\infty}\left\{\int_{-\infty}^{+\infty}\left(\int_{-\infty}^{+\infty}\left|g\left(x, y_{1}, y_{2}, z\right)\right|^{p_{1}} d y_{1}\right)^{p_{2} / p_{1}} d y_{2}\right\}^{p_{3} / p_{2}} d z\right)^{2 / p_{3}} \\
& \int_{\boldsymbol{R}^{5}}\left[A_{3}(x, y, z)\right]^{2} d x d y d z \\
& \leq c_{\delta, \varepsilon} \int_{\mathbb{R}^{2}}\left\{\int_{-\infty}^{+\infty}\left(\int_{-\infty}^{+\infty}\left[\int_{-\infty}^{+\infty}\left|g\left(x, y_{1}, y_{2}, z\right)\right|^{p_{1}} d y_{1}\right]^{p_{2} / p_{1}} d y_{2}\right)^{p_{3} / p_{2}} d z\right\}^{2 / p_{3}} d x .
\end{aligned}
$$

Les autres fonctions $G_{I, \delta, \varepsilon}$ s'estiment de la même façon. Les majorations portant sur $H_{\delta, \varepsilon}^{\prime}(g)$ sous les hypothèses $2^{\circ} \mathrm{b}$ de la proposition sont analogues; pour les lecteurs qui ne sont pas au courant de la théorie des classes de Hardy, on va indiquer les modifications.

b) On peut supposer $g$ assez, réguliére, par un argument de régularisation standard. Il s'agit alors d'estimer la norme $L^{2}\left(H_{n}\right)$ de la fonction $G_{I, \delta, \varepsilon}$ ci-dessus; on va encore supposer $n=2$ et $I=\{1\}$.

On pose

$$
G_{I, \delta, \varepsilon}^{1}(x, y, z)=\int_{-\infty}^{+\infty} \int_{-\infty}^{+\infty} \frac{g(x, y+t, z) d t_{1} d t_{2}}{\left|t_{1}\right|^{\left(1-\varepsilon_{1}+\delta\right) / 2}\left|t_{2}\right|^{\left(1+\varepsilon_{2}+\delta\right) / 2}}
$$


Alors d'après [2]:

$$
\begin{aligned}
& \int_{-\infty}^{+\infty}\left|A_{3}(x, y, z)\right|^{2} d z \\
& \quad \leq c_{\delta, \varepsilon}^{\prime}\left[\int_{-\infty}^{+\infty}\left(\int_{0}^{+\infty}\left|\frac{\partial p_{s}^{3}}{\partial s} * G_{I, \delta, \varepsilon}^{1}(x, y, z)\right|^{2} s d s\right)^{p_{3} / 2} d z\right]^{2 / p_{3}} .
\end{aligned}
$$

On utilise l'inégalité de Minkowski:

$$
\begin{aligned}
& \int_{\boldsymbol{R}^{2}}\left(\int_{-\infty}^{+\infty}\left|A_{3}(x, y, z)\right|^{2} d z\right) d y \\
& \quad \leq\left(\int_{-\infty}^{+\infty}\left[\int_{\boldsymbol{R}^{2}}\left(\int_{0}^{+\infty}\left|\frac{\partial p_{s}^{3}}{\partial s} * G_{I, \delta, \varepsilon}^{1}(x, y, z+i s)\right|^{2} s d s\right) d y\right]^{p_{3} / 2}\right)^{2 / p_{3}}
\end{aligned}
$$

On recommence le même argument en intégrant en $y_{1}$ et $y_{2}$ dans le second membre de l'inégalité ci-dessus; on trouve finalement que:

$$
\int_{\boldsymbol{R}^{5}}\left|A_{3}(x, y, z)\right|^{2} d x d y d z \leq c_{\delta, \varepsilon}\|\check{g}\|_{\vec{p}_{I}^{0}}^{2}=c_{\delta, \varepsilon}\|g\|_{\vec{p}_{I}^{0}} .
$$

On refait les mêmes estimations pour $A_{4}$. Ce qui termine la preuve de la proposition.

Cette proposition est démésurément longue, de plus, nous n'avons qu'esquissé les preuves un peu compliquées et nous sommes étendus sur les démonstrations faciles. On verra plus loin que pour établir l'existence d'une solution fondamentale les inégalités $1^{\circ}$ de la proposition suffisent. Mais les inégalités $2^{\circ}$ interviendront pour avoir des estimations globales des solutions $U$ de $P U=f$.

\section{Preuve du théorème I'.}

\section{Remarques.}

a) $1_{B}(x)\left|X_{J}\right|^{-1+\varepsilon} e^{+\delta\|\log x\|} \leq \sum_{I \subset J} \varphi_{I}^{0}(x) \varphi_{I^{c}}^{1}(x)\left|X_{I}\right|^{-(1-\varepsilon+\delta)}\left|X_{I^{c}}\right|^{-(1-\varepsilon-\delta)}$

b) $1_{B}(x)\left|X_{J}\right|^{-1+\varepsilon} e^{-\delta\|\log x\|} \geq \sum_{I \subset J} \varphi_{I}^{0}(x) \varphi_{I^{c}}^{1}(x)\left|X_{I}\right|^{-(1-\varepsilon-\delta)}\left|X_{I^{c}}\right|^{-(1-\varepsilon+\delta)}$

\section{Prouvons a):}

$$
\begin{aligned}
1_{B}(x)\left|X_{J}\right|^{-1+\varepsilon} e^{\delta\|\log x\|} & =1_{B}(x)\left|X_{J}\right|^{-1+\varepsilon} e^{\delta\|\log x\|} \sum_{j \in J}\left[\varphi_{J}^{0}\left(x_{j}\right)+\varphi_{j}^{1}\left(x_{j}\right)\right] \\
& =1_{B}(x)\left|X_{J}\right|^{-1+\varepsilon} e^{\delta\|\log x\|} \sum_{I \subset J} \varphi_{I}^{0}(x) \varphi_{I c}^{1}(x) .
\end{aligned}
$$


Mais

$$
\begin{aligned}
e^{\delta\|\log x\|} \varphi_{I}^{0}(x) \varphi_{I^{c}}^{1}(x) & \leq \exp \left(\delta \sum_{i \in I} \log \frac{1}{\left|x_{i}\right|}\right) \exp \left(\delta \sum_{i \in I} \log \left|x_{i}\right|\right) \varphi_{I}^{0}(x) \varphi_{I}^{1}(x) \\
& \leq\left|X_{I}\right|^{-\delta}\left|X_{I^{c}}\right|^{\delta} \varphi_{I}^{0}(x) \varphi_{I^{c}}^{1}(x) .
\end{aligned}
$$

Remarquons en fin que

c) Pour tout nombre réel $s$.

$$
\left[\sum_{I \subset J} \varphi_{I}^{0}(x) \varphi_{I^{c}}^{1}(x)\left|X_{I}\right|^{\alpha}\left|X_{I^{c}}\right|^{\beta}\right]^{s}=\sum_{I \subset J} \varphi_{I}^{0}(x) \varphi_{I^{c}}^{1}(x)\left|X_{I}\right|^{s \alpha}\left|X_{I c}\right|^{s \beta} .
$$

Pour tout $0<\delta<1 / 4 n$ et $\varepsilon$ satisfaisant les conditions de l'introduction (page 408) on pose:

$$
h_{\delta, \varepsilon}(\lambda, x)=\sum_{I \subset J} \varphi_{I}^{0}(x) \varphi_{I}^{1}(x)\left|X_{I}\right|^{(1-\varepsilon+\delta) / 2}\left|X_{I c}\right|^{(1-\varepsilon-\delta) / 2}|\lambda|^{\delta_{0}} .
$$

D’après les remarques a et $c$, $h_{\delta, \varepsilon} f$ est dans $L_{\delta / 2, \varepsilon}^{2}\left(\mathbb{R}^{n}\right)$ si $f \in L^{2}\left(\mathbb{R}^{n}\right)$. On peut donc lui appliquer la solution fondamentale $E_{\delta / 2, \varepsilon, \lambda}$ pour les valeurs $\delta / 2, \varepsilon$ des paramètres, construite à la proposition 4 pour l'opérateur $P_{\lambda}(D)$. On trouve alors une fonction $E_{\delta / 2, \varepsilon, \lambda} h_{\delta, \varepsilon} f \in L_{-\delta / 2, \varepsilon}^{2}\left(\mathbb{R}^{n}\right)$ dont la norme ne dépasse pas $|\lambda|^{\delta_{0}}\|f\|_{L^{2}\left(\boldsymbol{R}^{n)}\right)}$. Soient:

$$
\begin{aligned}
& f_{\delta, \varepsilon}(\lambda, x)=\sum_{I \subset J} \varphi_{I}^{0}(x) \varphi_{I^{c}}^{1}(x)\left|X_{I}\right|^{(1-\varepsilon-\delta) / 2}\left|X_{I c}\right|^{(1-\varepsilon+\delta) / 2}|\lambda|^{\delta_{0}} \\
& f_{\delta, \varepsilon}^{\prime}(\lambda, x)=\sum_{I \subset J} \varphi_{I}^{0}(x) \varphi_{I c}^{1}(x)\left|X_{I}\right|^{-(1-\varepsilon-\delta) / 2}\left|X_{I c}\right|^{-(1-\varepsilon+\delta) / 2}|\lambda|^{-\delta_{0}} .
\end{aligned}
$$

Définissons un opérateur de convolution $E_{\delta, \varepsilon}^{0}$ de $L^{2}\left(H_{n}\right)$ en imposant que sa transformée de Fourier vaut: $E_{\delta, \varepsilon, \lambda}^{0} g_{1}=f_{\delta, \varepsilon}(\lambda) E_{\delta / 2, \varepsilon, \lambda} h_{\delta}(\lambda) g_{1}$. C'est un opérateur borné sur $L^{2}\left(\boldsymbol{R}^{n}\right)$ dont la norme ne dépasse pas $1 / M$ par suite des remarques a), b) et c). Puisque $E_{\delta / 2, \varepsilon, \lambda}$ est une solution fondamentale de $P_{\lambda}(D)$ on a l'égalité:

$$
P_{\lambda} f_{\delta, \varepsilon} E_{\delta, \varepsilon, \lambda}^{0} g_{1}=h_{\delta} g_{1}
$$

au sens des distributions d'après la remarque $c$.

$1^{\circ}$ Nous allons transporter cette égalité à $H_{n}$. Nous avons déjà vu que $F_{\delta, \varepsilon}$ et $H_{\delta, \varepsilon}$ sont des distributions tempérèes sur $H_{n}$; leurs transformées de Fourier sont les opérateurs de multiplication par les fonctions $f_{\delta, \varepsilon}$ et $h_{\delta, \varepsilon}$.

Vérifions que $F_{\delta, \varepsilon} *\left(E_{\delta, \varepsilon}^{0} * g\right)$ est bien une distribution sur $H_{n}$ quand $g$ est dans $L^{2}\left(H_{n}\right)$. Soit $f$ une fonction de classe $C_{c}^{\infty}\left(\mathbb{R}^{n}\right)$, par définition 
a) $\left\langle F_{\delta, \varepsilon} * E_{\delta, \varepsilon}^{0} * g, f\right\rangle=\left\langle E_{\delta, \varepsilon}^{0} * g, f * \check{F}_{\delta, \varepsilon}\right\rangle$.

Alors $\left|\left\langle F_{\delta, \varepsilon} * E_{\delta, \varepsilon}^{0} * g, f\right\rangle\right| \leq\left\|E_{\delta, \varepsilon}^{0}\right\|\|g\|_{2}\left(\sum_{|s| \leq n+2}\left\|\left(1+\|x\|^{2}\right)^{n+1} D^{s} f\right\|_{L^{2}\left(\boldsymbol{H}_{n}\right)}^{2}\right)^{1 / 2}$ d'après la proposition 6 .

On veut vérifier que $P\left(F_{\delta, \varepsilon} * E_{\delta, \varepsilon}^{0} * f\right)=H_{\delta, \varepsilon} * f$ pour toute fonction de classe $C_{c}^{\infty}$. Soit $U_{l} \in C_{c}^{\infty}\left(H_{n}\right)$ une approximation de l'unité; il est facile de voir que: $U_{l} *\left(F_{\delta, \varepsilon} * E_{\delta, \varepsilon}^{0} * f\right)=\left(U_{l} * F_{\delta, \varepsilon}\right) *\left(E_{\delta, \varepsilon}^{0} * f\right)$. Puisque $U_{l} * F_{\delta, \varepsilon}$ est dans $L^{2}\left(H_{n}\right)$ d'après la proposition 6 , on voit que la transformée de Fourier de $U_{l} * F_{\delta, \varepsilon}$ pour chaque $\lambda$ vaut $\widehat{U}_{l}(\lambda) f_{\delta, \varepsilon}(\lambda)$ où $\widehat{U}_{l}$ est la transformée de Fourier de $U_{l}$. On considère aussi $U_{l} * F_{\delta, \varepsilon} * E_{\delta, \varepsilon} * f$ passage à la limite on voit que la transformée de Fourier de $F_{\delta, \varepsilon} *\left(E_{\delta, \varepsilon}^{0} * f\right)$ vaut $f_{\delta, \varepsilon}(\lambda) E_{\delta, \varepsilon, \lambda}^{0} \circ \widehat{f}(\lambda)$ qui est égale, par construction à $h_{\delta, \varepsilon} \widehat{f}(\lambda)$. Et l'égalité à prouver résulte de la proposition 2.

Soit $f$ une fonction $C_{c}^{\infty}\left(H_{n}\right)$, d'après la proposition $6(1) H_{\delta, \varepsilon}^{\prime} * f$ existe et elle est dans $L^{2}\left(H_{n}\right)$. On veut vérifier que $U=F_{\delta, \varepsilon} * E_{\delta, \varepsilon}^{0} * H_{\delta, \varepsilon}^{\prime} * f$ comme distribution et vérifie $P U=f$.

Mais d'après l'inégalité $1^{\circ}$ de la proposition $6, U$ est une distribution tempérée puisque pour toute $g \in C_{c}^{\infty}\left(H_{n}\right)$.

$2^{\circ} \quad\left|\left\langle F_{\delta, \varepsilon} * E_{\delta, \varepsilon}^{0} * H_{\delta, \varepsilon}^{\prime} * f, g\right\rangle\right| \leq c_{\delta, \varepsilon}\left\|E_{\delta, \varepsilon}^{0} * H_{\delta, \varepsilon}^{\prime} * f\right\|_{2}\left\|g * \breve{F}_{\delta, \varepsilon}\right\|_{2}$

$$
\begin{aligned}
& \leq c_{\delta, \varepsilon}\left\|H_{\delta, \varepsilon}^{\prime} * f\right\|_{2}\left(\sum_{|s| \leq n+2}\left\|\left(1+\|x\|^{2}\right)^{n} D^{s} g\right\|_{L^{2}\left(H_{n}\right)}^{2}\right)^{1 / 2} \\
& \leq c_{\delta, \varepsilon}\left(\sum_{|s| \leq 3 n+2}\left\|(1+\|x\|)^{3 n} D^{s} f\right\|_{2}^{2}\right)^{1 / p^{\prime}} \\
& \quad \times\left(\sum_{|s|<3 n+2}\left\|(1+\|(x, y, z)\|)^{3 n} f\right\|_{L^{1}\left(H_{n}\right)}\right)^{\left(p^{\prime}-2\right) / p^{\prime}} \\
& \quad \times\left(\sum_{|s| \leq n+2}\left\|\left(1+\|x\|^{2}\right)^{n} D^{s} g\right\|_{L^{2}\left(H_{n}\right)}^{2}\right)^{1 / 2}
\end{aligned}
$$

d'après les inégalités $1 \mathrm{a}$ ) et $1 \mathrm{~b}$ ) de la proposition 6 . Ce qui prouve bien que $F_{\delta, \varepsilon} * E_{\delta, \varepsilon}^{0} * H_{\delta, \varepsilon}^{\prime} * f$ est une distribution.

Il reste à vérifier la relation algébrique. Comme on l'a remarqué au début de la preuve, $H_{\delta, \varepsilon}^{\prime}$ est l'inverse de $H_{\delta, \varepsilon}$. D'après la construction précèdente on sait que $P U=H_{\delta, \varepsilon}^{\prime} * H_{\delta, \varepsilon}^{\prime} * f=f$. Ceci prouve que $F_{\delta, \varepsilon} * E_{\delta, \varepsilon}^{0}$ $* H_{\delta, \varepsilon}^{\prime}$ est une solution fondamentale de $P$.

$3^{\circ}$ Mettons en évidence une solution fondamentale pour $P^{*}$.

Par construction $E_{\delta / 2, \varepsilon, \lambda}$ satisfait l'equation $E_{\delta / 2, \varepsilon, \lambda} P_{\lambda} U=U$ pour des 
donctions raisonables $U$ sur $\mathbb{R}^{n}$.

D'autre part, d'après, la première partie, $E_{\delta / 2, \varepsilon, \lambda}$ n'est autre que la transformée de Fourier en $\lambda$ de la distribution $F_{\delta, \varepsilon} * E_{\delta, \varepsilon}^{0} * H_{\delta, \varepsilon}^{\prime}=E_{\delta, \varepsilon}$. Il s'en suit que

$$
E_{\delta, \varepsilon} P=I_{d} \quad \text { et } \quad P^{*} E_{\delta, \varepsilon}^{*}=I_{d}
$$

ce qu'il fallait prouver.

Démonstration du théorèm I. Pour prouver le théorème I il ne reste plus qu'à prouver la seconde assertion puisque la première a été prouvée dans le théorèm $I^{\prime}$.

On va donc supposer que les polynômes $q_{\alpha}(\lambda)=\sum_{1 \leq \alpha \leq I_{K}, r} C_{I_{K}}^{\alpha} a_{I_{K}, r}(-i \lambda)^{r}$ - $(-\lambda)^{\left|I_{K}\right|}$ ont des zéros communs. Soit $q_{p}$ leur plus grand commun diviseur. Alors les coefficients du polynôme $Q_{k} \mid q_{p}$ qui sont des polynômes en $\lambda$ n'ont plus de zéros communs. On peut donc faire les constructions du théorème $I^{\prime}$ pour $P_{\lambda} \mid q_{p}$. D'oú une solution fondamentale $E_{\delta / 2, \varepsilon, \lambda}$ pour cet opérateur et une distribution $E_{\delta, \varepsilon}$ sur $H_{n}$ de la forme $E_{\delta, \varepsilon}=F_{\delta, \varepsilon} * E_{\delta, \varepsilon}^{0}$ $* H_{\delta, \varepsilon}^{\prime}$ telle que

$$
P E_{\delta, \varepsilon} f=q_{p}\left(-i \frac{\partial}{\partial z}\right) f
$$

pour toute fonction $C_{c}^{\infty}\left(H_{n}\right)$.

La même observation est valable pour $P^{*}$. Soit $\Omega$ un ouvert relativement compact de $H_{n}$ et soit $V$ une distribution à support compact dans $\Omega$; considérons une solution fondamentale $S$ pour l'opérateur différentiel à coefficients constants $q_{p}\left(-i \frac{\partial}{\partial z}\right)$ qui soit une distribution tempérée et une fonction $\theta \in C_{c}^{\infty}\left(\mathbb{R}^{n}\right)$ qui vaut 1 sur un voisinage de $\Omega$; alors $\theta(S * V)$ est une distribution à support compact; soit $W=\left[F_{\delta, \varepsilon} * E_{\delta, \varepsilon}^{0} * H_{\delta, \varepsilon}^{\prime}\right.$ $* \theta(S * V)]_{\Omega}$ la restriction à $\Omega$ de la distribution $F_{\delta, \varepsilon} * E_{\delta, \varepsilon}^{0} * H_{\delta, \varepsilon}^{\prime} *[\theta(S * V)]$ alors il est facile de vérifier que $\langle P W, g\rangle=\langle V, g\rangle$ pour toute $g \in C_{c}^{\infty}(\Omega)$ :

Evidemment si $V$ est une fonction $C_{c}^{\infty}(\Omega), W$ est une fonction $C_{c}^{\infty}(\Omega)$. Par ailleurs (1) Chang a montré que $H_{n}$ est $P$-convexe pour tout opérateur différentiel $P$ invariant; il découle de $\left[5\right.$ Th. 1.9] que $P C^{\infty}=C^{\infty}$; ces arguments s'appliquent à $P^{*}$ et terminent la preuve du théorèm I. 
Corollaire 1. Soit $f \in L_{2, \vec{p}^{0}}\left(H_{n}\right) \cap L_{2, \vec{p}^{1}}\left(H_{n}\right)$ où $1<p_{i}<2$. Alors l'équation $P U=f$ a toujours une solution distribution $U$; pour toute partie finie $I \subset J$, il existe $\delta^{I}, \varepsilon^{I}$ et une distribution $U_{I}$ telle que:

a) $\quad U=\sum_{I \subset J} U_{I}$

b) $\quad \sum_{I C J}\left\|F_{\delta I, \varepsilon I^{\prime}}^{\prime} * U_{I}\right\|_{L^{2\left(H_{n}\right)}} \leq C\left(\|f\|_{2, \vec{p}^{0}}+\|f\|_{2, \vec{p}^{1}}\right)$

Preuve $d u$ Corollaire 1. On va considérer la fonction $f_{I}=\Phi_{I}^{0} * \Phi_{I c}^{1} * f$, pour toute partie finie $I \subset J$. On peut choisir $\delta^{I}$ assez petit de telle sorte que le vecteur $\varepsilon^{I}$ de $\mathbb{R}^{n}$ défini par:

$$
\begin{aligned}
& \forall i \in I, \quad \varepsilon_{i}^{I}=2 / p_{i}^{\prime}+\delta^{I} \\
& \forall i \notin I, \quad \varepsilon_{i}^{I}=2 / p_{i}^{\prime}-\delta^{I}
\end{aligned}
$$

satisfasse les inégalités:

$$
0<\varepsilon_{i}^{I}<1 ; \quad \varepsilon_{i}^{I}+\delta^{I}<1 ; \quad \varepsilon_{i}^{I}-\delta^{I}>0 .
$$

Ces choix de $\delta^{I}, \varepsilon^{I}$ sont possibles par les hypothèses du corollaire. On peut donc trouver une solution de $P U_{I}=f_{I}$ de la forme:

$$
U_{I}=F_{\delta I, \varepsilon r} * E_{\delta I, \varepsilon r}^{0} * H_{\delta r, \varepsilon r}^{\prime} * f_{I}
$$

d'après le théorème puisque:

$$
\begin{aligned}
\left\|H_{\delta, \varepsilon}^{\prime} * f_{I}\right\|_{2} & \leq c_{\delta I, \varepsilon I}\left(\|f\|_{2, \vec{p}_{I}^{1}}+\|f\|_{2, \vec{p}_{I}^{0}}\right) \\
& =c_{\delta I, \varepsilon I}\left(\|f\|_{2, \vec{p}^{1}}+\|f\|_{2, \vec{p}^{0}}\right) .
\end{aligned}
$$

Cette inégalité découle de la proposition 6 (2a) puisque $f=\sum_{I \subset J} f_{I}$. Par ailleurs $F_{\delta I, \varepsilon I} * g$ est une distribution tempérée pour toute fonction $g \in L^{2}\left(H_{n}\right)$ d'après la proposition 6 (1a). Puisque $F_{\delta I, \varepsilon I}^{\prime}$ est l'inverse de $F_{\delta I, \varepsilon I}$, la preuve est terminée.

On va énoncer un autre corollaire, dont la preuve est exactement la même que celle qu'on vient de faire, qui découlerait d'un analogue pour $\varepsilon=0$ du théorème $I^{\prime}$ et de la proposition $(6,2 . b)$.

Corollaire 2. Soit $f \in R_{\vec{p}^{0}} \cap R_{\vec{p}^{1}}, \quad 0<p \leq 1$. Alors l'équation $P U$ If a toujours une solution distribution $V$; pour toute partie finie $I$ $\subset J$, il existe $\delta^{I}, \varepsilon^{I}$ est une distribution $U_{I}$ telle que: 

a) $U=\sum_{I \subset J} U_{I}$
b) $\sum_{I \subset J}\left\|F_{\delta I, \varepsilon I}^{\prime} * U_{I}\right\|_{L^{2}\left(H_{n}\right)} \leq c\left(\|f\|_{\vec{p}^{0}}+\|f\|_{\vec{p}^{1}}\right)$.

Pour terminer ce paragraphe signalons qu'on peut préciser la régularité des $f$ telles que la solution $U$ de $P U=f$ trouvée ci-dessus soit par exemple une fonction continue.

\section{§ 3. Demonstration du Théorème $\mathbb{H}$}

La preuve de ce théorème ainsi que celle du théorème suivant utilisera quelques résultats sur les transformées de Fourier des inverses de pôlynômes.

Nous commencerons cependant par une description de la solution fondamentale et nous examinerons les problèmes de convergence d'intégrales soulevés au cours de cette étude dans les pages qui suivent.

1. Description de la solution fondamentale. On va commencer par introduire certaines fonctions et décrire la solution fondamentale comme la limite de ces fonctions dans un sens approprié.

Pour tout $\lambda \in \mathbb{R}$, notons $H_{\lambda}$ la transformée de Fourier de $\left[Q_{\lambda}(x)\right]^{-1}$ comme fonction de $x$. Soit $U$ une fonction $C_{c}^{\infty}\left(\mathbb{R}^{n}\right)$ à support disjoint des axes de coordonnées et soit $I \subset J$ une partie finie. On pose:

$$
E_{\lambda}^{I} U(x)=\mathbb{1}_{I}(x) \int_{\mathbb{R}^{n}} \widetilde{U}_{I}(t) H_{\lambda}\left[\psi_{I}^{-1}(x)-t\right] d t
$$

$E_{\lambda}^{I} U$ est bien définie car $H_{\lambda}$ donne par convolution un opérateur borné sur $L^{2}\left(\boldsymbol{R}^{n}\right)$. C'est d'ailleurs une fonction de classe $C^{\infty}$ dans le secteur où on l'a définie, qui tend vers 0 quand l'un des $x_{i}$ tend aussi vers 0 . $\mathrm{Si}$ on fait le changement de variables $t=\psi_{I}^{-1}(v)$ on trouve que

$$
E_{\lambda}^{I} U(x)=\int_{\left(\mathbb{R}_{+}\right)^{n}} U\left(v_{I},-v_{I c}\right) H_{\lambda}\left\{\log _{I}\left(\frac{x_{I}}{v_{I}}\right), \log \left(\frac{-x_{I c}}{v_{I^{c}}}\right)\right\} \frac{d v}{v} \mathbb{H}_{I}(x) .
$$

Dans cette expression $\left[\log _{I}\left(\frac{x_{I}}{v_{I}}\right), \log \left(\frac{-x_{I c}}{v_{I c}}\right)\right]$ désigne le point de $\mathbb{R}^{n}$ de coordonnées:

$$
\left\{\log \frac{x_{i_{1}}}{v_{i_{1}}}, \cdots, \log \frac{x_{i_{r}}}{v_{i_{r}}}, \log \left(\frac{-x_{i_{r+1}}}{v_{i_{r+1}}}\right), \cdots, \log \left(\frac{-x_{i_{n}}}{v_{i_{n}}}\right)\right\}
$$




$$
\frac{d v}{v}=\prod_{i=1}^{n} \frac{d v_{i}}{v_{i}} \text { et }\left\{v_{I},-v_{I c}\right\}=\left(v_{i_{1}}, \cdots, v_{i_{r}},-v_{i_{r+1}}, \cdots,-v_{i_{n}}\right)
$$

où $I=\left(i_{1}, \cdots, i_{r}\right) ; I^{c}=\left(i_{r+1}, \cdots, i_{n}\right)$.

Elle vaut aussi:

$$
\begin{aligned}
E_{\lambda}^{I} U(x)= & \mathbb{I}_{I}(x) \int_{\boldsymbol{R}^{|I|}} \int_{\boldsymbol{R}^{\prime I^{c \mid}}}\left(\mathbb{1}_{J}^{+} U\right)\left(v_{I},-v_{I c}\right) \\
& \times H_{\lambda}\left(\log \frac{x_{I}}{v_{I}}, \log \left(\frac{-x_{I c}}{v_{I c}}\right)\right) \frac{d v_{I^{c}}}{v_{I c}} \frac{d v_{I}}{v_{I}} \\
= & \mathbb{I}_{I}(x) \int_{R^{n}} U(v) \mathbf{1}_{I}^{+}\left(v_{I}\right) \mathbb{1}_{I c}^{+}\left(-v_{I c}\right) H_{\lambda}\left[\log \left(\frac{x}{v}\right)\right] \frac{d v}{v}
\end{aligned}
$$

Considérons l'opérateur $E_{\lambda}$ défini à priori sur les fonctions $U, C_{c}^{\infty}\left(\boldsymbol{R}^{n}\right)$ à support compact disjoint des axes par la formule:

$$
E_{\lambda} U(x)=\sum_{I \subset J} \mathbf{1}_{I}(x) E_{\lambda}^{I} U(x)
$$

qui, d'après le calcul antèrieur vaut aussi:

$$
E_{\lambda} U(x)=\int_{\boldsymbol{R}^{n}} U(v)\left\{\sum_{I \subset J} \mathbb{1}_{I}(x) \mathbf{1}_{I}^{+}\left(v_{I}\right) \mathbb{1}_{I^{c}}^{+}\left(-v_{I c}\right)\right\} H_{\lambda}\left(\frac{x}{v}\right) \frac{d v}{v} .
$$

Posons:

$$
K_{\lambda}(v, u)=\sum_{I \subset J} \mathbb{1}_{I}(v) \mathbb{1}_{I}^{+}\left(u_{I}\right) \mathbf{1}_{I^{c}}^{+}\left(-u_{I c}\right) H_{\lambda}\left[\log _{I}\left(\frac{v}{u}\right)\right],
$$

$K_{\lambda}$ n'est autre que le noyau de $E_{\lambda}$.

Si l'on prouve que $E_{\lambda}$ donne lieu à un opérateur borné $\operatorname{sur} L^{2}\left(\boldsymbol{R}^{n}\right)$ le même argument qu'à la preuve du théorème $I$ montrera que $E_{\lambda}$ est une solution fondamentale de $P_{\lambda}(\xi, D)$.

En fait on veut calculer la trace de $\Pi_{\lambda}(x, y, z) E_{\lambda}$ dont le noyau vaut

$$
\begin{aligned}
& {\left[\Pi_{\lambda}(x, y, z) K_{\lambda}\right](v, u)} \\
& \quad=e^{i \lambda(y \cdot v-z)} \sum_{I \subset J} \frac{\mathbf{1}_{I}(v-x) \mathbf{1}_{I}^{+}\left(u_{I}\right) \mathbf{1}_{I^{c}}^{+}\left(-u_{I c}\right) H_{\lambda}\left[\log _{I}((v-x) / u)\right]}{u} .
\end{aligned}
$$

En général sous les hypothèses du théorème cette trace n'existe pas toujours; pour éviter ces ennuis, on va régulariser $E_{k}$ correctement. Pour cela on va introduire deux fonctions $\omega_{\varepsilon^{\prime}}$ et $\theta_{\varepsilon, \varepsilon_{1}}$ dépendant de trois paramètres $\varepsilon, \varepsilon^{\prime}, \varepsilon_{1}$. 
$\omega(x)=\left(\varepsilon^{\prime}\right)^{-(n+1)} e^{-\|(x, t)\| / \varepsilon^{\prime}} ; \theta_{\varepsilon, \varepsilon_{1}}(x)=\prod_{j=1}^{j=n} \theta_{j}\left(x_{j}\right)$ où $\theta$ est la fonction caractéristique de l'ensemble $\left\{x_{j}, \varepsilon<\left|x_{j}\right|<1 / \varepsilon_{1}\right\}$.

Soit

$$
\begin{aligned}
K_{\lambda}^{\varepsilon, \varepsilon^{\prime}, \varepsilon_{1}}(v, u)= & \mathbb{1}_{I}(v) \sum_{I \subset J} \theta_{\varepsilon, \varepsilon_{1}}(u) \mathbb{1}_{I}^{+}\left(u_{I}\right) \\
& \times \frac{\mathbb{1}_{I^{c}}^{-}\left(u_{I c}\right) H * \omega_{\varepsilon^{\prime}}\left[\log _{I}(v / u)\right]\left(1+\varepsilon \lambda^{2}\right)^{-n}}{u}
\end{aligned}
$$

ou $\mathbb{1}_{I^{c}}(u)=\mathbb{1}_{I^{c}}^{+}(-u)$.

On va prouver qu'il existe une suite $E^{\varepsilon, \varepsilon^{\prime}, \varepsilon_{1}}$ d'opérateurs de convolution de $L^{2}\left(H_{n}\right)$, un opérateur de convolution $E$ de $L^{2}\left(H_{n}\right)$ tel que pour tout $\lambda$, la transformée de Fourier de $E^{\varepsilon, \varepsilon^{\prime}, \varepsilon_{1}}$ a pour noyau $K_{\lambda}^{\varepsilon^{\varepsilon} \varepsilon^{\prime}, \varepsilon_{1}}$, la norme de $E^{\varepsilon, \varepsilon^{\prime}, \varepsilon_{1}}$ comme opérateur sur $L^{2}\left(H_{n}\right)$ est dominée par une constante absolue $c ; E_{\lambda}^{\varepsilon, \varepsilon^{\prime}, \varepsilon_{1}}$ provient d'une fonction explicite sur $H_{n} ; E^{\varepsilon, \varepsilon^{\prime}, \varepsilon_{1}}$ converge fortement vers $E$.

Preuve de l'assertion précédente. Définissons

$$
\begin{aligned}
& E_{\lambda, \varepsilon_{1}}^{\varepsilon, \varepsilon^{\prime}, I} U(x)=\mathbb{1}_{I}(x) \\
& \quad=\mathbb{1}_{I}(x) \int_{\mathbb{R}^{n}} \mathbb{1}^{+}\left(v_{I^{c}}\right) \mathbb{1}_{I^{c}}\left(v_{I^{c}}\right) H_{\lambda} * \omega_{\varepsilon^{\prime}}\left[\log \left(\frac{x}{v}\right)\right] U(v) \theta_{\varepsilon, \varepsilon_{1}}(v) \frac{d v}{v} .
\end{aligned}
$$

Il est clair que pour tout couple $\left(U_{1}, U_{2}\right)$ de fonctions à supports compacts disjoints des axes de coordonnées

$$
\lim _{\substack{\varepsilon \rightarrow 0 \\ \varepsilon^{\prime} \rightarrow 0 \\ \varepsilon_{1} \rightarrow 0}}\left\langle E_{\lambda, \varepsilon_{1}}^{\varepsilon, \varepsilon^{\prime}} U_{1}, U_{2}\right\rangle=\left\langle E_{\lambda}^{I} U_{1}, U_{2}\right\rangle .
$$

Il est non moins clair que la norme de $E_{\lambda, \varepsilon_{1}}^{\varepsilon_{,}^{\prime}, I}$ comme opérateur sur $L^{2}\left(\boldsymbol{R}^{n}\right)$ est dominée par une constante indépendante de $\lambda$ fois celle de $H_{\lambda} * \omega_{\varepsilon}$, comme opérateur de convolution de $L^{2}\left(\mathbb{R}^{n}, e^{1 \cdot x} d x\right)$ que nous estimons ci-dessous.

On remarque aussi que

$$
H_{\lambda} * \omega_{\varepsilon^{\prime}}(x)=e^{-1 \cdot x / 2} \int_{\boldsymbol{R}^{n}} \frac{\widehat{\omega}_{\varepsilon^{\prime}}(\xi+\vec{i} / 2)}{Q(s+\vec{i} / 2, \lambda)} e^{i x \cdot \xi} d \xi
$$

où $\vec{i} / 2$ désigne le vecteur de $\boldsymbol{C}^{n}$ de composantes $i / 2$.

Ceci est une simple conséquence de la formule de Stokes et du fait 
que la fonction $\xi \mapsto \sup _{0 \leq y_{j} \leq 1 / 2} \frac{\left|\widehat{\omega}_{\xi^{\prime}}(\xi+i y)\right|}{|Q(\xi+i y, \lambda)|}$ tend vers zéros quand $\|\xi\| \rightarrow+\infty$.

On pose $H_{\lambda} * \omega_{\varepsilon^{\prime}}(x)=e^{-1 \cdot x / 2} H_{\varepsilon^{\prime}}^{1}(x)$. Par hypothèse $H_{\varepsilon^{\prime}}^{1}$ est un opérateur de convolution de $L^{2}\left(\boldsymbol{R}^{n}\right)$ dont la norme est dominée par une constante absolue, puisque $\forall \xi \in \mathbb{R}^{n}, \forall y, \quad 0 \leq y_{i} \leq 1 / 2, \mid Q(\xi+i y, \lambda) i \geq c$. Soit $g \in C_{c}^{\infty}\left(\boldsymbol{R}^{n}\right)$

$$
\begin{aligned}
H_{\lambda} * \omega_{\varepsilon^{\prime}} * g(x) & =e^{-1 \cdot x / 2} \int_{\boldsymbol{R}^{n}} H_{\varepsilon^{\prime}}^{1}(x-t) e^{1 \cdot t / 2} g(t) d t \\
\left\|H_{\lambda} * \omega_{\varepsilon^{\prime}} * g\right\|_{2,1} & =\left\|H_{\varepsilon^{\prime}}^{1} *\left(e^{1 \cdot t / 2} g\right)\right\|_{2} \\
& \leq c\|g\|_{2,1} .
\end{aligned}
$$

On voit done que $H_{\lambda} * \omega_{\varepsilon}$, définit un opérateur sur $L^{2}\left(\boldsymbol{R}^{n}, e^{1 \cdot x} d x\right)$ dont la norme ne dépasse pas une constante fixe.

On définit une fonction $f^{\varepsilon, \varepsilon^{\prime}, \varepsilon_{1}}$ sur $H_{n}$ par la relation

$$
\begin{aligned}
& f^{\varepsilon, \varepsilon^{\prime}, \varepsilon_{1}}(x, y, z) \\
& =\sum_{I \subset J} \int_{\boldsymbol{R}^{n+1}} \frac{e^{-i \lambda(y \cdot(U+x)-z)} \mathbf{1}_{I}(U+x) \theta_{\varepsilon, \varepsilon_{1}}(U) \mathbf{I}_{I}^{+}\left(U_{I}\right) \mathbf{1}_{I^{c}}^{-}\left(U_{I c}\right)}{\times H_{\lambda} * \omega_{\varepsilon^{\prime}}\left[\log _{I}((U+x) / U)\right]|\lambda|^{n} d \lambda d U} \\
& =\int_{\boldsymbol{R}^{n+1}} e^{-i \lambda(y \cdot(U+x)-z)} K_{\lambda}^{\varepsilon, \varepsilon^{\prime}}(U+x, U)|\lambda|^{n} d U d \lambda .
\end{aligned}
$$

Remarquons que $f^{\varepsilon, \varepsilon^{\prime}, \varepsilon_{1}}$ est bien définie, puisque $\theta_{\varepsilon}$ est à support compact disjoint des axes, que $H_{\lambda} * \omega_{\varepsilon}$, est bornée car $\left|Q_{\lambda}(\xi)\right|^{-1} \leq c$ et $\widehat{\omega}_{\varepsilon^{\prime}}$ est bien intégrable.

On va vérifier que la transformée de Fourier de $f^{\varepsilon} \varepsilon^{\varepsilon^{\prime}, \varepsilon_{1}}$ est le champ mesurable, borné, d'opérateurs $\lambda \mapsto E_{\lambda}^{\varepsilon_{1}} \varepsilon^{\prime}, \varepsilon_{1}$ :

$$
\begin{aligned}
\forall g \in C_{c}^{\infty}\left(\boldsymbol{R}^{n}\right), & \int_{\boldsymbol{R}^{2 n+1}} f^{\varepsilon, \varepsilon^{\prime}, \varepsilon_{1}}(x, y, z) \bar{g}(x, y, z) d x d y d z \\
& =\int_{-\infty}^{+\infty} \operatorname{Tr}\left[E^{\varepsilon, \varepsilon^{\prime}, \varepsilon_{1}} \circ \widehat{g} *\left(\Pi_{\lambda}\right)\right]|\lambda|^{n} d \lambda .
\end{aligned}
$$

Pour cela on fera deux remarques.

i) Soit $g$ une fonction de classe $C_{c}^{\infty}\left(H_{n}\right)$, si $\widehat{g}_{2,3}$ désigne la transformée de Fourier de $g$ en $(y, z)$ le noyau de $\widehat{g}\left(\Pi_{\lambda}\right)$ vaut $\hat{g}\left(\Pi_{\lambda}\right)(\xi, x)=$ $\widehat{g}_{2,3}(\xi-x,-\lambda \xi, \lambda)$ par conséquent celui de $\hat{g}\left(\Pi_{\lambda}\right) *$ est:

$$
\left[\widehat{g}\left(\Pi_{\lambda}\right)\right]^{*}(h, u)=\overline{\hat{g}}_{2,3}(u-h,-\lambda u, \lambda)
$$


ii) $g(x, y, z)=\int_{-\infty}^{+\infty} \operatorname{Tr}\left[\Pi_{\lambda}\left(h^{-1}\right) h\left(\Pi_{\lambda}\right)\right]|\lambda|^{n} d \lambda \quad$ où $\quad h^{-1}=(-x,-y$, $-z-x y)$. Ces deux remarques découlent directement de la description des répresentations $\Pi_{\lambda}$.

Soit à prouver l'assertion ci-dessus

$$
\begin{aligned}
& \int_{\boldsymbol{R}^{2 n+1}} f^{\varepsilon, \varepsilon^{\prime}, \varepsilon_{1}}(x, y, z) \bar{g}(x, y, z) d x d y d z \\
& \quad=\int_{\boldsymbol{R}^{2 n+1}}\left[\int_{\boldsymbol{R}^{n}} e^{i(y \cdot U-\lambda z)} K_{\lambda}^{\varepsilon, \varepsilon^{\prime}, \varepsilon_{1}}(U / \lambda,(U / \lambda)-x) d \lambda d U\right] \bar{g}(x, y, z) d y d x d z \\
& =\int_{\boldsymbol{R}^{2 n+1}} K_{\lambda}^{\varepsilon, \varepsilon^{\prime}, \varepsilon_{1}}(U, U-x) \overline{\hat{g}}_{2,3}(x,-\lambda u, \lambda)|\lambda|^{n} d x d u d \lambda \\
& =\int_{\boldsymbol{R}^{2 n+1}} K_{\lambda}^{\varepsilon, \varepsilon^{\prime}, \varepsilon_{1}}\left(\xi_{1}+x, \xi_{1}\right) \overline{\hat{g}}\left(x_{1},-\lambda\left(\xi_{1}+x\right), \lambda\right)|\lambda|^{n} d \xi_{1} d x d \lambda \\
& =\int_{\boldsymbol{R}^{2 n+1}} K_{\lambda}^{\varepsilon, \varepsilon^{\prime}, \varepsilon_{1}}\left(u, \xi_{1}\right) \overline{\hat{g}}_{2,3}\left(u-\xi_{1},-\lambda u, \lambda\right)|\lambda|^{n} d u d \xi_{1} d \lambda .
\end{aligned}
$$

D’après la formule

$$
\begin{aligned}
K_{\lambda}^{\varepsilon, \varepsilon^{\prime}, \varepsilon_{1}}\left[\widehat{g}\left(\Pi_{\lambda}\right)\right]^{*}(v, u) & =\int_{\mathbb{R}^{n}} K_{\lambda}^{\varepsilon, \varepsilon^{\prime}, \varepsilon_{1}}(v, h) \widehat{g}^{*}(\Pi)(h, u) d h \\
& =\int_{\mathbb{R}^{n}} K_{\lambda}^{\varepsilon, \varepsilon^{\prime}, \varepsilon_{1}}(v, h) \overline{\hat{g}}_{2,3}(u-h,-\lambda u, \lambda) d h .
\end{aligned}
$$

Par conséquent:

$$
\begin{aligned}
\int_{-\infty}^{+\infty} \operatorname{Tr}\left[K_{\lambda}^{\varepsilon, \varepsilon^{\prime}, \varepsilon_{1}} \circ \widehat{g}\left(\Pi_{\lambda}\right) *|\lambda|^{n} d \lambda\right. \\
\quad=\int_{\boldsymbol{R}^{2 n+1}} K_{\lambda}^{\varepsilon, \varepsilon^{\prime}, \varepsilon_{1}}(u, h) \overline{\widehat{g}}_{2,3}(u-h,-\lambda u, \lambda)|\lambda|^{n} d h d u d \lambda .
\end{aligned}
$$

Ce qui prouve l'égalité souhaitée.

Comme la norme des opérateurs $E^{\varepsilon, \varepsilon^{\prime}, \varepsilon_{1}}$ est bornée indépendamment de $\varepsilon, \varepsilon^{\prime} \varepsilon_{1}$ et qu'on a l'égalité (1) il s'ensuit que $E$ est la limite forte des opérateurs $E^{\varepsilon, \varepsilon^{\prime}, \varepsilon_{1}}$, par conséquent la distribution encore notée $E$ qui donne lieu à l'opérateur est la limite des fonctions $f^{\varepsilon, \varepsilon^{\prime}, \varepsilon_{1}}$ considerées ci-dessus.

On veut étudier cette limite. On va d'abord réécrire $f^{\varepsilon, \varepsilon^{\prime}, \varepsilon_{1}}$ sous une forme un peu plus agréable, en examinant chaque terme de la somme:

$$
f_{I}^{\varepsilon, \varepsilon^{\prime}, \varepsilon_{1}}(x, y, z)
$$




$$
\begin{aligned}
= & \int_{\boldsymbol{R}^{n}} e^{-i \lambda(y \cdot(U+x)-z)} \mathbf{1}_{I}(U+x) \theta_{\varepsilon, \varepsilon_{1}}(U) \mathbf{1}_{I}^{+}\left(U_{I}\right) \mathbf{1}_{I^{c}}^{-}\left(U_{I c}\right) \\
& \times H_{\lambda} * \omega_{\varepsilon^{\prime}}\left[\log \left(\frac{U+x}{U}\right)\right]|\lambda|^{n}\left(1+\varepsilon \lambda^{2}\right)^{-n} d \lambda d U \\
= & \int_{\boldsymbol{R}^{n}} \mathbf{1}_{\mathrm{I}}(U+x) \frac{\theta_{\varepsilon, \varepsilon_{1}}(U) \mathbf{1}_{I}^{+}\left(U_{I}\right) \mathbf{1}_{I^{c}}\left(U_{I^{c}}\right)}{U} \\
& \times \int_{\boldsymbol{R}^{2 n+1}} \frac{\widehat{\omega}_{\varepsilon^{\prime}}(\xi)|\lambda|^{n} \exp (-i(\lambda(y \cdot(U+x)-z)}{Q(\xi, \lambda)\left(1+\varepsilon^{\prime} \lambda^{2}\right)^{n}} \\
= & \int_{\boldsymbol{R}^{n}} \theta_{\varepsilon, \varepsilon_{1}}(U) \mathbf{1}_{I}^{+}\left(U_{I}\right) \mathbf{1}_{I^{c}}\left(U_{I c}\right) \\
& \times H^{\varepsilon^{\prime}}\left[\log _{I} \frac{U+x}{U}, y \cdot(U+x)-z\right] \mathbf{1}_{I}(U+x) \frac{d U}{U}
\end{aligned}
$$

où $H^{\varepsilon^{\prime}}$ désigne la transformée de Fourier de la fonction $\widehat{\omega}_{\varepsilon^{\prime}}(\xi)|\lambda|^{n} / Q(\xi, \lambda) \cdot\left(1+\varepsilon^{\prime} \lambda^{2}\right)^{-h}$ par rapport à la variable $(\xi, \lambda)$.

On doit faire face maintenant aux problèmes de convergence.

2. Problèmes de Convergence. Si $|\lambda|^{n}[Q(\xi, \lambda)]^{-1}$ est absolument sommable, pour tout $I \subset J$,

$$
\begin{aligned}
\lim _{\varepsilon^{\prime} \rightarrow 0} & \int_{\boldsymbol{R}^{n}} \theta_{\varepsilon, \varepsilon_{1}}(U) \mathbf{1}_{I}^{+}\left(U_{I}\right) \mathbf{I}_{I^{c}}^{-}\left(U_{I^{c}}\right) \\
& \times H^{\varepsilon^{\prime}}\left[\log _{I}\left(\frac{U+x}{U}\right), y \cdot(U+x)-z\right] \mathbf{1}_{I}(U+x) \frac{d U}{U} \\
= & \int_{\boldsymbol{R}^{n}} \theta_{\varepsilon, \varepsilon_{1}}(U) \mathbf{1}_{I}^{+}\left(U_{I}\right) \mathbf{1}_{I^{c}}^{-}\left(U_{I c}\right) \\
& \times H\left[\log _{I} \frac{U+x}{U}, y \cdot(U+x)-z\right] \mathbf{1}_{I}(U+x) \frac{d U}{U}
\end{aligned}
$$

ceci résulte simplement du fait que $H^{\varepsilon}$ converge uniformément vers la fonction $H^{0}$ et que $\theta_{\varepsilon, \varepsilon_{1}}$ est à support compact. Dans ce cas il ne reste plus qu'à étudier la limite:

$$
\begin{aligned}
& \lim _{\varepsilon_{1} \rightarrow 0} \int_{\boldsymbol{R}^{n}} \theta_{\varepsilon, \varepsilon_{1}}(U) \mathbf{1}_{I}^{+}\left(U_{I}\right) \mathbf{1}_{I^{c}}^{-}\left(U_{I c}\right) \\
& \quad \times H\left[\log _{I}\left(\frac{U+x}{U}\right), y \cdot(U+x)-z\right] \mathbb{1}_{I}(U+x) \frac{d U}{U}
\end{aligned}
$$


Les arguments qu'on vent développer pour étudier cette limite sont les même qu'on utilisera pour étudier la quantité analogue sous la seconde hypothèse; le lecteur voudra bien les suivre ci-dessous.

On aura besoin du lemme suivant sur les transformées de Fourier des inverses de polynômes.

Lemme 8. Soit $p$ assez grand; on considère la transformée de Fourier $H_{p}$ de la fonction $|\lambda|^{n} Q^{-1}\left(1+\lambda^{2}\right)^{-p}$. Soit $I \subset J$.

Sous les hypothèses $b$ du théorème II, pour p suffisamment grand, $H_{p}$ est une fonction et il existe une constante $c>0$ telle que si $\|x\| \geq c$ on $a$ :
c $) \quad\left|H_{p}(x, y)\right| \leq c_{1}$
$\left.c_{1}^{\prime}\right) \quad \operatorname{Sup}_{\varepsilon>0}\left|H_{p} * \omega_{\varepsilon^{\prime}}(x, t)\right| \leq c_{1}$
$\left.c_{2}\right) \quad\left|H_{p} * \omega_{\varepsilon^{\prime}}(x, t)\right| \leq c_{I} \exp \left(-\left(\sum_{i \in I} x_{i}\right) / 4\right) \exp \left(\varepsilon^{\prime} / 16\right)$.

Preuve du lemme 8. Il faut examiner deux cas:

i) $\mu \geq n+1$

ii) $\mu<n+1$.

Dans le premier cas, on pose $k=0$ et dans le second $k=n-\mu+1$. Il existe un polynôme $Q_{1}(\xi, \lambda)$ tel que $Q(\xi, \lambda)=Q_{1}(\lambda \xi, \lambda)$. Sous 1'hypothèse b) il existe un polynôme $q_{j}$ en $\lambda$ tel que:

$$
\left|\frac{\partial^{k}}{\partial \xi_{j}^{k}} Q_{1}^{-1}(\xi, \lambda)\right| \leq\left|q_{k_{j}}(\lambda)\right|(1+\|\xi\|)^{-\mu-k} .
$$

Il suffit de calculer les dériveés pour le voir. Soit $p$ tel que:

$$
c_{1}=\int_{-\infty}^{+\infty} \frac{|\lambda|^{k}\left(\sum_{j=1}^{n}\left|q_{k_{j}}(\lambda)\right|+1\right) d \lambda}{\left(1+|\lambda|^{2}\right)^{2 p}}<\infty .
$$

On voit tout de suite par intégration par parties que:

$$
\begin{aligned}
\|x\|^{k}\left|H_{p}(x, t)\right| & \leq c\left|\int_{-\infty}^{+\infty} \int_{\boldsymbol{R}^{n}} \frac{-\lambda^{k} e^{i t \lambda}}{\left(1+\lambda^{2}\right)^{p}} \sum_{j=1}^{n} \frac{\partial^{k}}{\partial \xi_{j}^{k}} Q_{1}^{-1}(\xi, \lambda) e^{-i x \xi / \lambda} d \xi d \lambda\right| \\
& \leq c \int_{\boldsymbol{R}^{n+1}} \frac{|\lambda|^{k}}{\left(1+\lambda^{2}\right)^{p}} \frac{\left(\sum_{j=1}^{n}\left|q_{k_{j}}(\lambda)\right|+1\right) d \xi d \lambda}{(1+\|\xi\|)^{\mu+k}} .
\end{aligned}
$$

Ce qui prouve $c_{1}$ puisque l'intégrale de droite est convergente; supposons 
$k \neq 0$.

On remarque aussi que la fonction $\xi \mapsto \sum_{j=1}^{n} \int_{\boldsymbol{R}^{n}} \frac{\partial^{k}}{\partial \xi_{j}^{k}} Q_{1}^{-1}(\xi, \lambda) e^{-i x \cdot \xi / \lambda} d \xi$ s'annule quand $x=0$, on peut donc $y$ remplacer $e^{-i x \cdot \xi / \lambda}$ par $e^{-i x \cdot \xi / \lambda}-1$ dont la valeur absolue ne dé passe pas $c\|x\|^{n}\|\xi\|^{\eta}|\lambda|^{-\eta}$, pour tout $0<\eta<1$. Par conséquent:

$$
\|x\|^{k}\left|H_{p}(x, t)\right| \leq c \int_{\boldsymbol{R}^{n+1}} \frac{|\lambda|^{k-\eta}\left(\sum_{j=1}^{n}\left|q_{k_{j}}(\lambda)\right|+1\right)\|\xi\|^{\eta}\|x\|^{\eta} d \xi d \lambda}{\left(1+\lambda^{2}\right)^{p}(1+\|\xi\|)^{\mu+k}} .
$$

Ce qui prouve que: $|H(x, t)| \leq c\|x\|^{-k+\eta}$. Considérons la fonction:

$$
\begin{aligned}
& \sup _{s>0} s^{-(n+1)} \int_{\|(y, t)\| \leq s}\left|H_{p}\left(x-y, t-t^{\prime}\right)\right| d y d t^{\prime} \\
\leq & \sup _{s>0} s^{-(n+1)} \int_{\|y\| \leq s} \int_{|t| \leq s}\left|H_{p}\left(x-y, t-t^{\prime}\right)\right| d y d t^{\prime} \\
\leq & c_{1} \sup _{s>0} s^{-n} \int_{\|y\| \leq s}\|x-y\|^{-k+\eta} d y
\end{aligned}
$$

si $k$ est non nul, on voit facilement que la quantité ci-dessus ne dépasse pas:

$$
c^{\prime}\|x\|^{-k+\eta}+c_{2}^{\prime}
$$

D'où $c_{1}$ et $c_{1}^{\prime}$ par les arguments classiques

Il reste à prouver $c_{2}$.

Soit $I \subset J$, on désignera par $(i / 4)_{I}$ le vecteur de $C^{n}$ tel que $x_{j}=i / 4$ si $j \in I$ et $x_{j}=0$ sinon.

D'après la formule de Stokes,

$$
\begin{aligned}
& H_{p} * \omega_{\varepsilon^{\prime}}(x, \tau) \\
& \quad=\exp \left(-\left(\sum_{j \in I} x_{j}\right) / 4\right) \int_{\boldsymbol{R}^{n+1}}|\lambda|^{n} \frac{e^{i(x, \tau) \cdot(\xi, \lambda)} \widehat{\omega}_{\xi^{\prime}}\left(\xi+(i / 4)_{I}\right) d \xi d \lambda}{Q\left(\xi+(i / 4)_{I}, \lambda\right)\left(1+\lambda^{2}\right)^{p}}
\end{aligned}
$$

et

$$
\begin{aligned}
& \exp \left(\left(\sum_{j \in I} x_{j}\right) / 4\right)\left(H_{p} * \omega_{\varepsilon^{\prime}}\right)(x, \tau) \\
& =H_{p, \varepsilon^{\prime}}^{1}(x, \tau)=\int_{\boldsymbol{R}^{n+1}} \frac{|\lambda|^{n} e^{i(x, \tau) \cdot(\xi, \lambda)} \widehat{\omega}_{\varepsilon^{\prime}}\left[\xi+(i / 4)_{I}\right] d \xi d \lambda}{Q\left(\xi+(i / 4)_{I}, \lambda\right)\left(1+\lambda^{2}\right)^{p}}
\end{aligned}
$$

fonction pour la quelle on veut faire le raisonnement précédent. 
Soit $\quad H_{p, \varepsilon^{\prime}}^{I}(x, \tau)=\int_{\boldsymbol{R}^{n+1}} \frac{|\lambda|^{n} e^{i(x, \tau) \cdot(\xi, \lambda)} d \xi d \lambda}{Q\left(\xi+(i / 4)_{I}, \lambda\right)\left(1+\lambda^{2}\right)^{p}}$.

D'après la forme explicite de $\widehat{\omega}_{\varepsilon^{\prime}}$, on voit facilement que:

$$
H_{p, \varepsilon^{\prime}}^{1}(x, \tau)=e^{\varepsilon^{\prime} / 16} H_{p}^{I} * \omega_{\varepsilon^{\prime}}\left(x-(\varepsilon / 8)_{I}, \tau\right) .
$$

Un raisonnement analogue à celui de la première partie prouve que si $\|x\|$ est assez grand

$$
\left|H_{p}^{I} * \omega_{\varepsilon^{\prime}}\left(x-(\varepsilon / 8)_{I}, \tau\right)\right| \leq\left\{\begin{array}{lll}
c & \text { si } & k=0 \\
|x|^{-k+\eta} & \text { si } & k \geq 1
\end{array}\right.
$$

ce qui prouve l'inégalité $c_{2}$.

Fin de la preuve du théorème II. Montrons d'abord que sous les hypothèses (b) du théorème.

$$
\begin{aligned}
\lim _{\varepsilon^{\prime} \rightarrow 0} & \int_{\boldsymbol{R}^{n}} \theta_{\varepsilon, \varepsilon_{1}}(U) \mathbb{1}_{I}^{+}\left(U_{I}\right) \mathbb{1}_{I^{c}}\left(U_{I c}\right) \\
& \times H_{p}^{\varepsilon^{\prime}}\left[\log _{I}\left(\frac{U+x}{U}\right), y \cdot(U+x)-z\right] \mathbb{1}_{I}(U+x) \frac{d U}{U} \\
= & \int_{\boldsymbol{R}^{n}} \theta_{\varepsilon, \varepsilon_{1}}(U) \mathbf{1}_{I}^{+}\left(U_{I}\right) \mathbf{1}_{I^{c}}\left(U_{I c}\right) \\
& \times H_{p}\left[\log _{I}\left(\frac{U+x}{U}\right), y \cdot(U+x)-z\right] \mathbb{H}_{I}(U+x) \frac{d U}{U}
\end{aligned}
$$

où $H_{p}^{\varepsilon^{\prime}}=H_{p} * \omega_{\varepsilon^{\prime}}$.

Ce sera une conséquence du lemme précédent et du théorème de convergence dominée de Lebesgue. Les cas embêtants sont ceux où l'on se trouve au voisinage d'un point $u$ avec l'un des $u_{i}=-x_{i}$. Nous étudions en détail l'exemple du groupe $H_{6}$; le cas général se traite de la même façon mais prend inutilement trop d'espace avec les notations. On examinera l'intégrale au voisinage du point $u_{1}=-x_{1} ; u_{2}=-x_{2} ; u_{3}=-x_{3}$; $u_{4} \neq-x_{4} ; u_{5} \neq-x_{5} ; u_{6} \neq-x_{6}$.

Soient $\alpha_{1}, \alpha_{2}, \alpha_{3}$ trois nombres positifs assez petits; on va decouper $\boldsymbol{R}^{3}$ à l'aide des $\alpha_{j}$ suivant des régions où l'on peut contrôler $H_{p}$.

On note

1) $A_{i, j, k}^{(1)}$ le produit de trois intervalles dont l'une des bornes seulement 
est finie et vaut $-x_{i}-\alpha_{i}$, ou $-x_{i}+\alpha_{i} ;-x_{j}+\alpha_{j}$ ou $-x_{j}-\alpha_{j} ;-x_{k}-\alpha_{k}$ ou $-x_{k}+\alpha_{k}$.

2) $A_{i, k}^{(2)}$ le produit de trois intervalles tels que:

a) l'une seule des bornes des deux premiers est finie et vaut

$$
-x_{i}-\alpha_{i} \text { ou }-x_{i}+\alpha_{i}
$$

b) le $3^{\circ}$ est de la forme $\left[-x_{k}-\alpha_{k},-x_{k}+\alpha_{k}\right]$.

3) $A_{i, j, k}^{(3)}$ est de la forme $T_{i}^{1} \times T_{j}^{2} \times T_{k}^{3}$ avec

$$
\left.\left.T_{i}^{1}=\right]-\infty,-x_{i}-\alpha_{i}\right] \text { ou }\left[-x_{i}+\alpha_{i},+\infty[\right.
$$

et

$$
T_{j}^{2}=\left[-\alpha_{j}-x_{j}, \alpha_{j}-x_{j}\right] ; T_{k}^{3}=\left[-\alpha_{k}-x_{k}, \alpha_{k}-x_{k}\right]
$$

4) $A_{1,2,3}^{(4)}=\left[-x_{1}-\alpha_{1},-x_{1}+\alpha_{1}\right] \times\left[-x_{2}-\alpha_{2},-x_{2}+\alpha_{2}\right]$

$$
\times\left[-x_{3}-\alpha_{3},-x_{3}+\alpha_{3}\right] \text {. }
$$

Alors

$$
\begin{aligned}
& \int_{\boldsymbol{R}^{\mathrm{B}}} \theta_{\varepsilon, \varepsilon_{1}}(U) \mathbf{1}_{I}^{+}\left(U_{I}\right) \mathbb{1}_{I^{c}}^{-c}\left(U_{I^{c}}\right) \\
& \quad \times H_{p}^{\varepsilon^{\prime}}\left[\log _{I}\left(\frac{U+x}{U}\right), y \cdot(U+x)-z\right] \mathbf{1}_{I}(U+x) \frac{d U}{U} \\
& =\sum_{i, j, k, l} \int_{A_{i, j, k}^{(1)} \times \boldsymbol{R}^{3}} \theta_{\varepsilon, \varepsilon_{1}}(U) \mathbb{1}_{I}^{+}\left(U_{I}\right) \mathbf{1}_{I^{c}}^{-c}\left(U_{I^{c}}\right) \\
& \quad \times H_{p}^{\varepsilon^{\prime}}\left[\log _{I}\left(\frac{U+x}{U}\right), y \cdot(U+x)-z\right] \mathbf{1}_{I}(U+x) \frac{d U}{U} .
\end{aligned}
$$

L'intégrale sur $A_{i, j, k}^{(1)} \times \mathbb{R}^{3}$ ne pose pas de problèmes puisque $H_{p}^{\varepsilon^{\prime}}\left[\log \frac{U+x}{U}\right.$, $y \cdot(U+x)-z]$ converge vers $H_{p}\left[\log _{I}\left(\frac{U+x}{U}\right), y \cdot(U+x)-z\right]$, de plus elle est bornée sur ces intervales d'après le lemme $8\left(c_{1}^{\prime}\right)$. Puisque $\theta_{\varepsilon, \varepsilon_{1}}$ est à support compact, le théorème de convergence dominée de Lebesgue montre que

$$
\begin{aligned}
& \lim _{\varepsilon^{\prime} \rightarrow 0} \sum_{i, j, k} \int_{A_{i, j, k}^{(1)} \times \boldsymbol{R}^{s}} \theta_{\varepsilon, \varepsilon_{1}}(U) \mathbf{1}_{I}^{+}\left(U_{I}\right) \mathbf{1}_{I^{c}}^{-}\left(U_{I^{c}}\right) \\
& \quad \times H_{p}^{\varepsilon^{\prime}}\left[\log \left(\frac{U+x}{U}\right), y \cdot(U+x)-z\right] \mathbf{1}_{I}(U+x) \frac{d U}{U} \\
& =\sum_{i, j, k} \int_{A_{i, j, k}^{(1)} \times \boldsymbol{R}^{\mathrm{s}}} \theta_{\varepsilon, \varepsilon_{1}}(U) \mathbf{1}_{I}^{+}\left(U_{I}\right) \mathbf{1}_{I^{c}}\left(U_{I^{c}}\right)
\end{aligned}
$$




$$
\times H_{p}\left[\log _{I}\left(\frac{U+x}{U}\right), y \cdot(U+x)-z\right] \mathbb{H}_{I}(U+x) \frac{d U}{U} .
$$

C'est la même preuve que sous l'hypothèse $a$.

Les intégrales sur les trois types d'intervalles se traitent de la même façon on peut étudier pas exemple celui de $A_{i, j, k}^{(3)}$.

Si $\alpha_{j}$ et $\alpha_{k}$ sont assez petits $\log \left(\frac{u_{2}+x_{2}}{u_{2}}\right), \log \left(\frac{u_{3}+x_{3}}{u_{3}}\right)$ sont assez grands et d'après le lemme $8, c_{2}$

$$
\left|H_{p}^{\varepsilon^{\prime}}\left[\log _{I}\left(\frac{U+x}{U}\right), y \cdot(U+x)-z\right]\right| \leq c\left|\frac{u_{2}}{u_{2}+x_{2}}\right|^{1 / 4}\left|\frac{u_{3}}{u_{3}+x_{3}}\right|^{1 / 4} \cdot \quad *
$$

On utilise encore le théorème de convergence dominée de Lebesgue pour conclure grâce à la compacité du support de $\theta_{\varepsilon, \varepsilon_{1}}$ que

$$
\begin{aligned}
& \lim _{\varepsilon^{\prime} \rightarrow 0} \int_{A_{i, j, k}^{(3)} \times \boldsymbol{R}^{\mathbf{8}}} \theta_{\varepsilon, \varepsilon_{1}}(U) \mathbb{1}_{I}^{+}\left(U_{I}\right) \mathbb{1}_{I^{c}}^{-}\left(U_{I^{c}}\right) \\
& \quad \times H_{p}^{\varepsilon^{\prime}}\left[\log _{I}\left(\frac{U+x}{U}\right), y \cdot(U+x)-z\right] \mathbb{1}_{I}(U+x) \frac{d U}{U} \\
& =\int_{A_{i, j, k}^{(3)}} \theta_{\varepsilon, \varepsilon_{1}}(U) \mathbb{1}_{I}^{+}\left(U_{I}\right) \mathbb{1}_{I c}\left(U_{I^{c}}^{-}\right) \\
& \quad \times H_{p}\left[\log _{I}\left(\frac{U+x}{U}\right), y \cdot(U+x)-z\right] \mathbb{1}_{I}(U+x) \frac{d U}{U} .
\end{aligned}
$$

Il reste à se débarrasser de $\varepsilon_{1}$.

On utilise encore l'inégalité $*$.

Le théorème de convergence dominée de Lebesgue permet encore de conclure que:

$$
\begin{aligned}
\lim _{\varepsilon_{1} \rightarrow 0} & \int_{\boldsymbol{R}^{\varepsilon}} \theta_{\varepsilon, \varepsilon_{1}}(U) \mathbb{1}_{I}^{+}\left(U_{I}\right) \mathbb{1}_{I^{c}}^{-}\left(U_{I^{c}}\right) \\
& \times H_{p}\left[\log _{I}\left(\frac{U+x}{U}\right), y \cdot(U+x)-z\right] \mathbb{1}_{I}(U+x) \frac{d U}{U} \\
= & \int_{\left|u_{i}\right|<1 / \varepsilon} \mathbb{1}_{I}^{+}\left(U_{I}\right) \mathbb{H}_{I^{c}}\left(U_{I^{c}}\right) \\
& \times H_{p}\left[\log _{I}\left(\frac{U+x}{U}\right), y \cdot(U+x)-z\right] \mathbb{H}_{I}(U+x) \frac{d U}{U} .
\end{aligned}
$$

Ce qui prouve le théorème sous l'hypothèse (a) avec $p=0$. Soit $p$ assez grand pour que les conditions du lemme 8 soient remplies et soit $H_{p}$ 
la fonction obtenue, pour terminer la preuve du théorème il faut d'abord remarquer, d'après ce qui a été dit antérieurement que

$$
\begin{aligned}
E_{0}= & \lim _{\varepsilon \rightarrow 0} \int_{\left|u_{i}\right| \leq 1 / \varepsilon} \sum_{I \subset J} \mathbf{1}_{I}^{+}\left(U_{I}\right) \mathbf{1}_{I^{c}}^{-}\left(U_{I c}\right) \\
& \times H_{p}\left[\log _{I}\left(\frac{U+x}{U}\right), y \cdot(U+x)-z\right] \mathbb{1}_{I}(U+x) \frac{d U}{U}
\end{aligned}
$$

existe en tant que distribution et la limite a lieu au sens des distributions. D'autre part si l'on désigne par $I_{d}$ la masse de Dirac à l'origne, il est clair d'après la construction qu'on a faite que:

$$
P\left[I_{d}-\left(\frac{\partial}{\partial z}\right)^{2 p}\right] E_{0}=I_{d}
$$

Pas conséquent:

$$
E=\left[I_{d}-\left(\frac{\partial}{\partial z}\right)^{2 p}\right] E_{0}
$$

est une solution fondamentale de $P$. Ce qui termine la preuve du théorème II.

\section{§ 4. Quelques Exemples d'Opérateurs Satisfisant les Hypothèses des Théorèmes II et III}

$1^{\circ}$ A chaque opérateur $\xi^{k}\left(d^{k} / d \xi^{k}\right)$ correspond, comme on l'a vu au paragraphe (I) un polynôme $P_{k}(\xi)$ de dégré $k$.

D'autre part, il est facile de vérifier que l'ensemble $\left\{1, \cdots, P_{k}\right\}$ forme une base de l'espace vectoriel complexe des polynômes de degré inférieur ou égal à $k$. Comme il en est ainsi de l'ensemble $\left(1, \xi, \cdots, \xi^{k}\right)$, pour chaque indice $\alpha$

$$
\xi^{\alpha}=\sum_{K \subset \alpha} p_{K}^{\alpha} P_{K}(\xi)
$$

où

i) $p_{K}^{\alpha}$ sont des constantes

ii) $P_{K}(\xi)=\prod_{k \in K} P_{K}\left(\xi_{k}\right)$.

Par conséquent le polynôme $(\lambda \xi)^{\alpha}$ correspondant à l'opérateur 


$$
P^{\alpha}=\sum_{K \subset \alpha} p_{K}^{\alpha}(i)^{\alpha} Y^{K} X^{K} Z^{|\alpha|-|K|}
$$

En particulier nous noterons $P_{i}^{2 m}$ l'opérateur correspondant à $\left(\lambda \xi_{i}\right)^{2 m}$

$2^{\circ}$ Soient $\left\{a_{i}\right\}_{i=1}^{n}$ des constantes strictement positives; considèrons l'opérateur

$$
P=\sum_{i=1}^{n} a_{i} P_{i}^{2 m}+q^{m}(i Z)
$$

où $q$ est un polynôme réel positif tel que $q(i \lambda) \geq 8 \lambda^{2} n+1$.

Alors suivant les valeurs de $m, P$ satisfait les hypothèses $a$ et $b$ du théorème II et celles de l'opérateur $P_{0}$ du théorème III.

La polynôme associé à $P$ est:

$$
Q(\lambda \xi, \lambda)=\sum_{j=1}^{n} a_{j}\left(\lambda \xi_{j}\right)^{2 m}+q^{m}(\lambda) .
$$

Si $q(\lambda) \geq 8 \lambda^{2} n$

$$
|Q((\lambda(\xi+i \eta)), \lambda)| \geq(3 / 8)^{m}\left[(\lambda\|\xi+i \eta\|)^{2}+q(\lambda)\right]^{m}
$$

pour tout $\|\eta\| \leq 1$.

Si $m \leq \frac{n}{2}+1$ les hypothèses a) du théorème II sont satisfaites; sinon les hypothèses $b$ du théorème II le sont; cet opérateur peut jouer le rôle de l'opérateur $P_{0}$ du théorème III.

$3^{\circ}$ Soit $Q^{0}$ un polynôme réel, elliptique de degré $2 m$; soit $v \in \mathbb{R}^{n}, 0 \leq v_{i}$ $\leq 1 / 2$; on note

$$
Q_{\alpha}^{0}(\eta)=D^{\alpha} Q^{0}(\eta)
$$

Soit:

$$
P_{0}=\sum_{\alpha} P^{\alpha}(X, Y) Q_{\alpha}^{0}(v Z)+i q^{m}(i Z)
$$

si on prend $\theta(\lambda)=v$ alors l'opérateur $P_{0}$ satisfait les bonnes hypothèses $\mathrm{du}$ théorème III pour un bon choix du polynôme réel $q(\lambda)$.

En effet le polynôme associé à $P_{0}$

$$
Q(\lambda \xi, \lambda)=Q^{0}(\lambda(\xi-i v))+i q^{m}(\lambda)
$$

et

$$
Q(\lambda(\xi+i v), \lambda)=Q^{0}(\lambda \xi)+i q^{m}(\lambda)
$$


Par définition du polynôme elliptique, il existe $a>0$ tel que si $\|x\| \geq a$, $\left|Q^{0}(\xi)\right| \geq c\|\xi\|^{2 m}$; soit $q$ un polynôme réel positif, $q(\lambda)>a$ pour tout $\lambda$

$$
\left(Q^{0}(\lambda \xi)\right)^{2}+[q(\lambda)]^{2 m} \geq c^{2}\|\lambda \xi\|^{4 m}+q^{2 m}(\lambda) \geq c_{1}\left[\|\lambda \xi\|^{2}+q(\lambda)\right]^{2 m}
$$

si $q(\lambda) \geq 8 n \lambda^{2}$, on aura l'inégalité désirée.

$4^{\circ}$ Si l'on pose:

$$
T(Y, Z)=\sum_{\alpha}(i)^{\alpha} Y^{\alpha} Q_{\alpha}^{0}(v Z)+i q^{m}(i Z)
$$

on voit aussi que $T$ est un bon candidat pour l'opérateur du théorème III, suivant les valeurs de $m$.

Preuve du théorème III.

A) $P_{\lambda}^{\omega}(\xi, D)$ s'exprime:

$$
\begin{aligned}
P_{\lambda}^{\omega}(\xi, D)= & \widehat{T}(i \lambda \xi-i \lambda \omega, \lambda) \sum_{I_{K}, r} a_{I_{K}, r}(-i \lambda)^{\left|I_{K}\right|}(-i \lambda)(\xi-\omega)^{\left|I_{K}\right|} \frac{\partial^{I_{K}}}{\partial \xi^{I_{K}}} \\
& +\sum_{\substack{|p|+i s \mid \leq m \\
r \leq p}}(-i \lambda)^{s}(i \lambda)^{|p|} b_{p, r, s}(\xi-\omega)^{p}(-1)^{|r|} \frac{\partial^{r}}{\partial \xi^{r}} .
\end{aligned}
$$

Par un changement de variables, on est ramené à l'opérateur correspondant à $\omega=0$.

$$
\begin{aligned}
P_{\lambda}(\xi, D)= & \widehat{T}(i \lambda \xi, \lambda) \sum_{I_{K}, r} a_{I_{K}, r}(-i \lambda)^{\left|I_{K}\right|+r} \xi^{I_{K}} \frac{\partial^{I_{K}}}{\partial \xi^{I_{K}}} \\
& +\sum_{\substack{|p|+|s| \leq m \\
r \leq p}}(-i \lambda)^{s}(-1)^{|r|} b_{p, r, s}(i \lambda)^{|p| \xi^{p}} \frac{\partial^{r}}{\partial \xi^{r}} .
\end{aligned}
$$

Soit $I \subset J$ une partie, on pose:

$$
T^{I}(\lambda u, \lambda)=\widehat{T}\left(i \lambda \psi_{I}(u), \lambda\right)
$$

et l'opérateur qu'on va étudier s'exprime:

$$
\begin{aligned}
P_{\lambda, I}^{0}(u, D)= & T^{I}(\lambda u, \lambda) \sum_{I_{K}, r} a_{I_{K}, r}(-i \lambda)^{r}(-i \lambda)^{\left|I_{K}\right|} \sum_{1 \leq \alpha \leq I_{K}} C_{I_{K}}^{\alpha} D^{\alpha} \\
& +\sum_{\substack{|p|+|r| \leq m \\
r \leq p}}(-i \lambda)^{s}(-1)^{|r|} b_{p, r, s}(i \lambda)^{|p-r|} \psi_{I}^{p-r}(u)(i \lambda)^{|r|} B_{r}(D)
\end{aligned}
$$

où

$$
B_{r}(D)=\sum_{1 \leq \alpha_{k} \leq r_{k}} C_{r}^{\alpha} D^{\alpha}
$$


Pour trouver un inverse de $P_{\lambda, I}^{0}$ il suffira de calculer un inverse de $R_{\lambda}^{I}(u, D)$ qui est $\left(T^{I}\right)^{-1} P_{\lambda, I}^{0}(u, D)$.

Plan de la démonstration. On fixe $u$ et l'on considère $R_{\lambda}^{I}(u, D)$ comme un opérateur différentiel à coefficients constants.

Sous les hypothèses du théorème on va prouver qu'il existe une solution fondamentale $\mathcal{E}_{\lambda}^{I}$ de $R_{\lambda}^{I}$ qui satisfait certaines inégalités.

Soit $S_{\lambda}^{I}$ l'opérateur défini sur $\mathscr{S}\left(\boldsymbol{R}^{n}\right)$ par la formule:

$$
S_{\lambda}^{I} f(t)=\int_{\boldsymbol{R}^{n}} \mathcal{E}_{\lambda}^{I}(t, t-y) f(y) d y .
$$

Soit $G_{\lambda}^{I}=R_{\lambda}^{I}(u, D) S_{\lambda}^{I}-I_{d}$.

On prouvera que la norme de $G_{\lambda}^{I}$ est strictement plus petite que un. Alors l'opérateur $G_{\lambda}^{I}+I_{d}$ sera inversible et l'égalité

$$
R_{\lambda}^{I}(u, D) S_{\lambda}^{I}\left(G^{I}+I_{d}\right)^{-1}=I_{d}
$$

fournira un inverse à gauche pour $R_{\lambda}^{I}$.

B) Estimations de $R_{\lambda}^{I}(u, \xi)$ et de ses dérivées.

Soit

$$
B(u, \xi+i v, \lambda)=\sum_{\substack{|p|+|r| \leq m \\ r \leq p}}(-i \lambda)^{s}(-1)^{|r|}(i \lambda)^{|p-r|} b_{p, r, s} \psi_{I}^{p-r}(u)(i \lambda)^{|r|} B_{r}(\xi+i v)
$$

on va commencer par estimer $\left(T^{I}\right)^{-1}(\lambda u, \lambda) B(u, \xi+i v, \lambda)$.

$\mathrm{B}_{1}$ ) On note

$$
\begin{aligned}
A_{p, r, s}^{I}=( & -i \lambda)^{r}(-1)^{|r|} b_{p, r, s}(i \lambda)^{|p-r|} \phi_{I}^{p-r}(u)(i \lambda)^{|r|} \\
& \times B_{r}(\xi+i v)\left(T^{I}\right)^{-1}(\lambda u, \lambda) .
\end{aligned}
$$

Soient $\quad r_{i} \leq p_{i}$ éventuellement nuls tels que: $\left[\psi_{I}^{p_{2}-r_{2}}(u)\right]|\lambda|^{p_{2}-r_{2}} \leq 1$; $\left[\psi_{I}\right](u)^{p_{1}-r_{1}}|\lambda|^{\left|p_{1}-r_{1}\right|} \geq 1$.

Puisque $\quad\left|T^{I}(\lambda u, \lambda)\right| \geq\left[\left\|\lambda \psi_{I}(u)\right\|+\left|q_{0}(\lambda)\right|\right]^{m+\beta}$

$$
\begin{aligned}
& \geq c_{1}\left|q_{0}(\lambda)\right|^{\beta}\left[\left\|\lambda \psi_{I}(u)\right\|+q_{0}(\lambda)\right]^{m} \\
& \geq c_{1}\left|q_{0}(\lambda)\right|^{\beta}\left|\lambda^{\left|p_{1}-r_{1}\right|} \psi_{I}^{p_{1}-r_{1}}(u)\right|\left(q_{0}(\lambda)\right)^{m-\left|p_{1}-r_{1}\right|} .
\end{aligned}
$$

Puisque $p_{1}+r_{1} \leq m$

$$
\left|A_{p, r, s}^{I}\right| \leq c|\lambda|^{s}\left|b_{p, r, s}\right|\left(1+|\lambda|^{|r|-1}\right)(\|\lambda(\xi+i v)\|+1)^{|r|}\left|q_{0}(\lambda)\right|^{-\beta}
$$


finalement:

a) si $|\lambda| \leq 1$

(1) $\left|\left(T^{I}\right)^{-1}(\lambda u, \lambda) B(u, \lambda, \xi+i v)\right|$ $\leq c \sum_{p, r, s}\left|q_{0}(\lambda)\right|^{-\beta}\left(\|\lambda(\xi+i v)\|^{2}+1\right)^{\left|r_{0}\right| / 2}|\lambda|^{s}\left|b_{p, r, s}\right|$.

$\left.a_{2}\right) \quad$ si $|\lambda| \geq 1$

(2) $\left|\left(T^{I}\right)^{-1}(\lambda u, \lambda) B(u, \lambda, \xi+i v)\right|$ $\leq c_{2}^{\prime}\left|q_{0}(\lambda)\right|^{-\beta} \sum_{p, r, s}\left|b_{p, r, s}\right||\lambda|^{s+|r|-1}\left(\|\lambda(\xi+i v)\|^{2}+1\right)^{\left|r_{0}\right| / 2}$.

Soit

$$
c_{1}(\lambda)=\left\{\begin{array}{lll}
\left|q_{0}(\lambda)\right|^{-\beta} \sum_{p, r, s}\left|b_{p, r, s}\right||\lambda|^{s} & \text { si } & |\lambda| \leq 1 \\
\left|q_{0}(\lambda)\right|^{-\beta} \sum_{p, r, s}\left|b_{p, r, s}\right||\lambda|^{s+|r|} & \text { si } & |\lambda| \geq 1
\end{array}\right.
$$

Puisque:

$$
\begin{aligned}
& \left|Q_{\lambda}(\lambda(\xi+i v))\right| \geq c\left[\|\lambda(\xi+i v)\|^{2}+q(\lambda)\right]^{\mu} \\
& \left|R_{\lambda}^{I}(u, \xi+i v)\right| \geq c\left[\|\lambda(\xi+i v)\|^{2}+q(\lambda)\right]^{\mu}-c_{2}^{\prime} c_{1}(\lambda)\left(\|\lambda(\xi+i v)\|^{2}+1\right)^{\left|r_{0}\right| / 2}
\end{aligned}
$$

si $c$ est grand par rapport à $c_{2}^{\prime} c_{1}(\lambda)$ on a l'inégalité:

$$
\left|R_{\lambda}^{I}(u, \xi+i v)\right| \geq\left[\|\lambda(\xi+i v)\|^{2}+q(\lambda)\right]^{\mu}\left[c-c_{1}(\lambda) c_{2}^{\prime}\right] .
$$

On veut déduire de (1), (2) et (3) des estimées sur les dérivées de $R_{\lambda}^{I}(u, \xi+i v)$; on va commencer par les dérivées de $T^{I}$.

$\mathrm{B}_{2}$ ) Estimations de dérivées de $T^{I}$.

$$
T^{I}(\lambda u, \lambda)=\sum_{\alpha, r} t_{\alpha, r}\left(i \lambda \psi_{I}(u)\right)^{\alpha}(-i \lambda)_{r}
$$

et:

$$
D_{u}^{r} T^{I}(\lambda u, \lambda)=\sum_{\alpha, r} t_{\alpha, \tau} \alpha^{r}\left(i \lambda \psi_{I}(u)\right)^{\alpha}(-i \lambda)^{r}
$$

Mais

$$
\left|T^{I}(\lambda u, \lambda)\right| \geq c\left[\left\|\lambda \psi_{I}(u)\right\|+q_{0}(\lambda)\right]^{m+\beta} \geq c\left\|\lambda \psi_{I}(u)\right\|^{|\alpha|}\left[q_{0}(\lambda)\right]^{m+\beta-|\alpha|}
$$

et:

(4) $\left|D_{u}^{r} T^{I}(\lambda u, \lambda)\left(T^{I}\right)^{-1}(\lambda u, \lambda)\right| \leq c \sum_{\substack{\alpha \neq 0 \\ r}} \frac{\left|t_{\alpha, r}\right| \alpha^{r}|\lambda|^{r}}{\left|q_{0}(\lambda)\right|^{m+\beta-|\alpha|}}=c c_{1}(\lambda, T)$. 
Soient $H^{0}=\left(T^{I}\right)^{-1} B(u, \lambda, \xi+i v)$ et $p \neq 0$. Alors

$$
D_{u}^{p} R_{\lambda}^{I}=D_{u}^{p}\left(H^{0}\right)
$$

et

$$
D_{u}^{p}\left(H^{0}\right)=\left(D_{u}^{p} B-\sum_{p \Varangle \alpha}\left(\begin{array}{c}
p \\
\alpha
\end{array}\right) D_{u}^{\alpha} H^{0} D_{u}^{p-a} T^{I}\right)\left(T^{I}\right)^{-1}
$$

On obtient, comme dans (1) et (2) l'inégalité:

$$
\left|D_{u}^{p} B\left(T^{I}\right)^{-1}(\xi+i v)\right| \leq\left(\|\lambda(\xi+i v)\|^{2}+1\right)^{\mid r_{0}: / 2} c_{1}(\lambda) .
$$

Par conséquent:

(5)

$$
\begin{aligned}
& \left|D_{u}^{p}\left(H^{0}\right)(\xi+i v)\right| \\
& \quad \leq c c_{1}(\lambda, T) \sum_{\alpha<p}\left|D_{u}^{\alpha} H^{0}(\xi+i v)\right|+c_{1}(\lambda)(\|\lambda(\xi+i v)\|+1)^{\left|r_{0}\right| / 2} \\
& \mid p_{1}^{\mid}=1 \text { des inégalités (2) et (5) on déduit la suivante: }
\end{aligned}
$$

$$
\left|D_{u}^{p} H^{0}(\xi+i v)\right| \leq c c_{1}(\lambda)\left[1+c_{1}(\lambda, T)\right]\left(\|\lambda(\xi+i v)\|^{2}+1\right)^{\mid r_{0^{\prime}} / 2} .
$$

Il s'en suit que pour tout $p$

$$
\left|D_{u}^{p} H^{0}(\xi+i v)\right| \leq c q_{p}^{\prime}\left[c_{1}(\lambda, T)\right] c_{1}(\lambda)\left(\|\lambda(\xi+i v)\|^{2}+1\right)^{\left|r_{0}\right| / 2} .
$$

où $q_{p}^{\prime}$ est un polynôme d'une variable.

On suppose l'assertion vraie pour $|p|=l$ et voici la preuve pour $|p|=l+1$. D'après (5)

$$
\begin{aligned}
& \left|D_{u}^{p} H^{0}(\xi+i v)\right| \\
& \quad \leq c\left(c_{1}(\lambda, T) \sum_{\alpha<p} q_{p-1}^{\prime}\left[c_{1}(\lambda, T)\right]+1\right) c_{1}(\lambda)\left(\|\lambda(\xi+i v)\|^{2}+1\right)^{\left|r_{0}\right| / 2} .
\end{aligned}
$$

On remarque que les polynômes $q_{p}^{\prime}$ sont de degrés croissants en $|p|$. On aura besoin de calculer des dérivées $D_{u}^{p} R_{\lambda}^{I}$ d'ordre élèvè mais fixe. On aura donc un polynôme $q_{T}$ indépendant de $|p| \leq M$ tel que:

$$
\left|D_{u}^{p} R_{\lambda}^{I}(\xi+i v)\right| \leq c c_{1}(\lambda) q_{r}\left[c_{1}(\lambda, T)\right]\left(\|\lambda(\xi+i v)\|^{2}+1\right)^{\left|r_{0}\right| / 2}
$$

on pose $q^{0}(\lambda)=c_{1}(\lambda) q_{T}\left[c_{1}(\lambda, T)\right]$.

La prochaine étape consiste à étudier les dérivées de $\left(R_{\lambda}^{I}\right)^{-1}=V_{\lambda}$ en $\xi$ et $u$.

$\left.\mathrm{B}_{3}\right)$ Estimation des dérivées de $V_{\lambda}$.

Exprimons la dérivée d'ordre $p$ de $V_{\lambda}$ en termes de celles de $R_{\lambda}^{I}$ :

$$
D_{u}^{p} V_{\lambda}(u, \xi+i v)
$$




$$
=\sum_{|\alpha| \leq|p|-1}\left(\begin{array}{c}
p \\
\alpha
\end{array}\right) D_{u}^{\alpha} V_{\lambda}(u, \xi+i v) D_{u}^{p-\alpha} R_{\lambda}^{I}(u, \xi+i v) V_{\lambda}(\xi+i v) .
$$

À une constante multiplicative près, d'après (6)

(7) $\left|D_{u}^{p} V_{\lambda}(u, \xi+i v)\right|$

$$
\leq c \sum_{|\alpha| \leq|p|-1}\left|D_{u}^{\alpha} V_{\lambda}(u, \xi+i v)\right| q^{0}(\lambda)\left(\|\lambda(\xi+i v)\|^{2}+1\right)^{\left|r_{0}\right| / 2}\left|\left(V_{\lambda}(u, \xi+i v)\right)\right|
$$

et d'après (3)

$$
\leq c \sum_{|\alpha| \leq|p|-1}\left|D_{u}^{a} V_{\lambda}(u, \xi+i v)\right| q^{0}(\lambda) .
$$

Si l'on a supposé dans (3) que $\left(c-c_{1}(\lambda) c_{2}^{\prime}\right)>c_{3}$ et que $\left|r_{0}\right| \leq 2 \mu$ comme il a été dit dans l'énoncé du théorème.

Un raisonnement analogue à celui de la fin de $B_{2}$ montre que:

$$
\left|D_{u}^{p} V_{\lambda}(u, \xi+i v)\right| \leq c q^{0}(\lambda) s_{1}(\lambda)\left[\|\lambda(\xi+i v)\|^{2}+q(\lambda)\right]^{-\mu}
$$

où $s_{1}$ est un polynôme en $q^{0}(\lambda)$ qu'on peut fixer si l'on calcule des dérivées d'ordre inférieur à un nombre donné.

On veut alors estimer:

$$
\begin{aligned}
D_{\xi}^{k} D_{u}^{p} R_{\lambda}^{I}(u, \xi+i v)= & \left(T^{I}\right)^{-1}\left\{D_{u}^{p}\left[D_{\xi}^{k}(B(u, \lambda, \xi))\right]\right. \\
& \left.-\sum_{\alpha \leq p} D_{u}^{\alpha} D_{\xi}^{k}\left(H^{0}\right) D_{u}^{p-\alpha} T^{I}\right\}
\end{aligned}
$$

si $|p| \neq 0$

$$
D_{\xi}^{k} D_{u}^{p} R_{\lambda}^{I}(u, \xi+i v)=D_{\xi}^{k} D_{u}^{p} H^{0}(u, \xi+i v) .
$$

Par conséquent un argument semblable au précèdent montre que:

$$
\left|D_{\xi}^{k} D_{u}^{p} R_{\lambda}^{I}(u, \xi+i v)\right| \leq c q^{0}(\lambda)\left[\|\lambda(\xi+i v)\|^{2}+|q(\lambda)|\right]^{\left|r_{0}-k\right| / 2}
$$

si $|k| \leq\left|r_{0}\right|$.

Avec la notation de page 401

$\left(9^{\prime}\right) \quad\left|D_{\xi}^{k} R_{\lambda}^{I}(u, \xi+i v)\right| \leq\left[\|\lambda(\xi+i v)\|^{2}+q(\lambda)\right]^{\mu-\mid k: / 2} q_{2}(\lambda) \quad 0<|k| \leq 2 \mu$

si on réécrit la dérivée d'ordre $k$ de $V_{\lambda}$ en tenant compte de (1) et $\left(9^{\prime}\right)$, on trouve:

$$
\begin{aligned}
& \left|D_{\xi}^{k} V_{\lambda}(u, \xi+i v)\right| \\
& \quad \leq c \sum_{|\alpha| \leq|k|-1}\left|D_{\xi}^{\alpha} V_{\lambda}(\xi+i v)\right|\left|D_{\xi}^{k-\alpha} R_{\lambda}^{I}(u, \xi+i v)\right|\left|V_{\lambda}(u, \xi+i v)\right|
\end{aligned}
$$




$$
\begin{gathered}
\left.\leq c \sum_{|\alpha|<|k|-1}\left|D_{\xi}^{\alpha} V_{\lambda}(\xi+i v)\right|\left[\|\lambda(\xi+i v)\|^{2}+1\right)\right]^{\mu-|k-\alpha| / 2} \\
\times q_{2}(\lambda)\left[\|\lambda(\xi+i v)\|^{2}+q(\lambda)\right]^{-\mu} \\
\leq c \sum_{|\alpha| \leq|k|-1}\left|D_{\xi}^{\alpha} V_{\lambda}(\xi+i v)\right|\left(\|\lambda(\xi+i v)\|^{2}+1\right)^{-|k-\alpha| / 2} q_{2}(\lambda) .
\end{gathered}
$$

Cette inégalité entraine que:

$$
\begin{aligned}
& \left|D_{\xi}^{k} V_{\lambda}(u, \xi+i v)\right| \\
& \quad \leq\left[q_{2}(\lambda)\right]^{|k|}\left[\|\lambda(\xi+i v)\|^{2}+q(\lambda)\right]^{-\mu}\left(\|\lambda(\xi+i v)\|^{2}+1\right)^{-|k| / 2} .
\end{aligned}
$$

Dans la dernière étape, on va estimer les dérivées mixtes de $V_{\lambda}$.

$\left.\mathrm{B}_{4}\right)$ Estimation des dérivées mixtes de $V$.

$$
\begin{aligned}
& D_{\xi}^{k} D_{u}^{p} V_{\lambda}(u, \xi+i v) \\
& \begin{aligned}
&=\sum_{\substack{\alpha<p \\
k_{1} \leq k \\
k_{2} \leq k_{1}}}\left(\begin{array}{l}
p \\
\alpha
\end{array}\right)\left(\begin{array}{l}
k \\
k_{1}
\end{array}\right)\left(\begin{array}{l}
k_{1} \\
k_{2}
\end{array}\right) D_{\xi}^{k_{2}} D_{u}^{\alpha} V_{\lambda}(u, \xi+i v) D_{\xi}^{k_{1}-k_{2}} D_{u}^{p-\alpha} R_{\lambda}^{I}(u, \xi+i v) \\
& \times D_{\xi}^{k-k_{1}} V_{\lambda}(u, \xi+i v) .
\end{aligned}
\end{aligned}
$$

Par conséquent d'après (9) et (10)

$$
\begin{aligned}
& \left|D_{\xi}^{k} D_{u}^{p} V_{\lambda}(u, \xi+i v)\right| \\
& \quad \leq c \sum_{\substack{\alpha<p \\
k_{1} \leq k \\
k_{2} \leq k k_{1}}}\left|q_{2}(\lambda)\right|^{\left|k-k_{1}\right|}\left(\|\lambda(\xi+i v)\|^{2}+1\right)^{-\left|k_{1}-k_{2}\right| / 2} q^{0}(\lambda) \\
& \quad \times\left|D_{\xi}^{k_{2}} D_{u}^{\alpha} V_{\lambda}(u, \xi+i v)\right|
\end{aligned}
$$

si $|p|=1$, d'après (1) cette inégalité entraine la suivante:

$$
\begin{aligned}
\left|D_{\xi}^{k} D_{u}^{p} V_{\lambda}(u, \xi+i v)\right| \leq & c q^{0}(\lambda)\left|q_{2}(\lambda)\right|^{|k|}\left[\|\lambda(\xi+i v)\|^{2}\right. \\
& +q(\lambda)]^{-\mu}\left(\|\lambda(\xi+i v)\|^{2}+1\right)^{-|k| / 2} .
\end{aligned}
$$

Il s'en suit qu'on peut trouver deux constantes $c_{M_{0}}^{1}, c_{M_{0}}^{2}$ ne dépendant que de $M_{0},|k| \leq M_{0},|p| \leq M_{0}$ telles que:

$$
\begin{aligned}
& \left|D_{\xi}^{k} D_{u}^{p} V_{\lambda}(u, \xi+i v)\right| \\
\leq & \frac{\left[c_{M_{0}}^{1}\left(q^{0}(\lambda)\left|q_{2}(\lambda)\right|^{|k|}\right)^{|p|}+c_{M_{0}}^{2} q^{0}(\lambda)\left[q_{2}(\lambda)\right]^{|k|}\right]\left(\|\lambda(\xi+i v)\|^{2}+1\right)^{-|k| / 2}}{\left[\|\lambda(\xi+i v)\|^{2}+q(\lambda)\right]^{\mu}} .
\end{aligned}
$$

Ce qui termine les estimations sur les dérivées.

C) Estimations des Transformées de Fourier $\int_{\mathbb{R}^{n}} e^{i x \cdot \xi}(i \lambda)^{\alpha-p-\beta}$ $\times D_{t}^{D} V_{\lambda}(t, \xi+i \theta(\lambda) / 2) d \xi=F_{\alpha, \beta, p}(t, x)$.

Dans toute cette partie on suppose $0<p \leq \alpha ; 0 \leq \beta \leq \alpha-p$ et le coefficient 
de $D^{\alpha}$ dans $Q_{\lambda}(D)$ où $B(u, \lambda, D)$ non nul.

On suppose de plus que le polynôme $Q_{\lambda}$ est de degré $2 \mu$ en $\xi$ et satisfait une inégalité du type:

$$
\left|Q_{i}[\lambda(\xi+i v)]\right| \geq c\left[\|\lambda(\xi+i v)\|^{2}+q(\lambda)\right]^{\mu}
$$

pour $v$ tel que

$$
\|\theta(\lambda) / 2-v\| \leq \eta
$$

où $\eta$ est un nombre positif ou nul; on veut trouver deux constantes $c_{2}(\lambda, b), c_{3}(\lambda, b)$, telles que:
a) $\left|\int_{\boldsymbol{R}^{n}}(i \xi)^{\alpha-p-\beta} e^{i \xi \cdot x} D_{t}^{p} V_{\lambda}[t, \xi+i \theta(\lambda) / 2] d \xi\right|$

$$
\leq c_{2}(\lambda, b)\|x\|^{-n+(1 / 2)} e^{-\eta\|x\|} \quad \text { si } \quad\|x\| \leq a
$$
b) $\left|\int_{\boldsymbol{R}^{n}}(i \xi)^{\alpha-p-\beta} e^{i \xi \cdot x} D_{t}^{p} V_{\lambda}[t, \xi+i \theta(\lambda) / 2] d \xi\right|$

$$
\leq c_{3}(\lambda, b)\|x\|^{-n-1} e^{-\eta\|x\|} \quad \text { si } \quad\|x\| \geq a .
$$

$\mathrm{C}_{1}$ ) On examine d'abord le cas où $\eta=0$.

On va faire deux estimations:

i) $n+1 \leq 2 \mu-|\alpha-p-\beta|$.

D’après (8)

$$
\begin{aligned}
& \int_{\boldsymbol{R}^{n}}\|\xi\|^{|\alpha-p-\beta|}\left|D_{t}^{p} V_{\lambda}(u, \xi+i \theta(\lambda) / 2)\right| d \xi \\
& \quad \leq q^{0}(\lambda) s_{1}(\lambda) \int_{\boldsymbol{R}^{n}} \frac{\|\xi\|^{|\alpha-p-\beta|} d \xi}{\left[(\lambda\|\xi\|)^{2}+q(\lambda)\right]^{\mu}} \\
& \quad=c q^{0}(\lambda) s_{1}(\lambda)[q(\lambda)]^{-\mu+n / 2+|\alpha-p-\beta| / 2}\left[|\lambda|^{-2 \mu+1}+|\lambda|^{-(n+1)}\right]
\end{aligned}
$$

et l'on a l'estimation à prouver au voisinage de 0 .

ii) $2 \mu-|\alpha-p-\beta| \leq n$; on pose $s=n-2 \mu+|\alpha-p-\beta|+1$.

D'après (11)

$$
\begin{aligned}
& \left.|| x_{i}\right|^{s} \int_{\boldsymbol{R}^{n}}(i \xi)^{\alpha-p-\beta} D_{u}^{p} V_{\lambda}(u, \xi+i \theta(\lambda) / 2) e^{i \xi \cdot x} d \xi \mid \\
& \quad=\left|\int_{\boldsymbol{R}^{n}} \sum_{k=0}^{s}\left(\begin{array}{l}
s \\
k
\end{array}\right)(i \xi)^{\alpha-p-\beta-k} D_{u}^{p} D_{i}^{s-k} V_{\lambda}(u, \xi+i \theta(\lambda) / 2)\left(e^{i \xi \cdot x}-1\right) d \xi\right|
\end{aligned}
$$




$$
\begin{aligned}
& \leq \sum_{k=0}^{s} c_{M_{0}}^{1}\left[q^{0}(\lambda) q_{2}(\lambda)^{s-k}\right]^{|p|}+c_{M_{0}}^{2} q^{0}(\lambda)\left[q_{2}(\lambda)^{s-k}\right] \\
& \times \int_{R^{n}} \frac{\|\xi\|^{|\alpha-p-\beta|-k-1 / 2} d \xi}{\left[\|\lambda \xi\|^{2}+q(\lambda)\right]^{\mu+s / 2}} \\
&=c \sum_{k=0}^{s}\left[c_{M_{0}}^{1}\left[q^{0}(\lambda)\left[q_{2}(\lambda)\right]^{s-k}\right]^{|p|}+c_{M_{0}}^{2} q^{0}(\lambda)\left[q_{2}(\lambda)\right]^{s-k}\right] \\
& \times|x|^{\mid 1 / 2}|q(\lambda)|^{-1 / 2}|\lambda|^{-\left(n+1 / 2+\mid \alpha-p-\beta_{i}-k\right)} .
\end{aligned}
$$

Par conséquent:

$$
\begin{aligned}
& \left|\int_{\boldsymbol{R}^{n}}(i \xi)^{\alpha-p-\beta} D_{u}^{p} V_{\lambda}(u, \xi+i \theta(\lambda) / 2) e^{i \xi \cdot x} d \xi\right| \\
& \quad \leq\|x\|^{-n+1 / 2+2 \mu-|\alpha-p-\beta|+1}|q(\lambda)|^{-1 / 2}\left(|\lambda|^{-2 \mu+1 / 2}+|\lambda|^{-n-2 \mu+1 / 2}\right) \\
& \quad \times \sum_{k=0}^{s}\left(c_{M_{0}}^{1}\left[q^{0}(\lambda)\left[q_{2}(\lambda)\right]^{s-k}\right]^{|p|}+c_{M_{0}}^{2} q^{0}(\lambda)\left[q_{2}(\lambda)\right]^{s-k}\right) .
\end{aligned}
$$

iii) On pose $k_{0}=n+1$; on travaille alors comme en ii) et l'on voit que:

$$
\begin{aligned}
& \left|x_{i}\right|^{k_{0}}\left|\int_{\boldsymbol{R}^{n}}(i \xi)^{\alpha-p-\beta} D_{u}^{p} V_{\lambda}(u, \xi+i \theta(\lambda) / 2) e^{i \xi \cdot x} d \xi\right| \\
& \leq c_{M_{0}}^{1} \sum_{k_{1}=0}^{k_{0}}\left[q^{0}(\lambda)\left[q_{2}(\lambda)\right]^{k_{0}-k_{1}}\right]^{|p|}+c_{M_{0}}^{2} q^{0}(\lambda)\left[q_{2}(\lambda)\right]^{k_{0}-k_{1}} \\
& \quad+c_{M_{0}}^{2} q^{0}(\lambda)\left[q_{2}(\lambda)\right]^{k_{0}-k_{1}} q(\lambda)^{-\mu+(|\alpha-\beta-p|-1) / 2}|\lambda|^{-\left(n+\mid \alpha-\beta-p_{1}-k_{1}\right)} .
\end{aligned}
$$

Par conséquent:

$$
\begin{aligned}
& \left|\int_{\boldsymbol{R}^{n}}(i \xi)^{\alpha-p-\beta} D_{u}^{p} V_{\lambda}(u, \xi+i \theta(\lambda) / 2) e^{i \xi \cdot x} d \xi\right| \\
& \quad \leq\|x\|^{-n-1}\left(|\lambda|^{-2 \mu+1}+|\lambda|^{-n-2 \mu+1}\right)|q(\lambda)|^{-\mu+(|\alpha-p-\beta|-1) / 2} \\
& \quad \times \sum_{k_{1}=0}^{k_{0}} c_{M_{0}}^{1}\left[q^{0}(\lambda)\left[q_{2}(\lambda)\right]^{k_{0}-k_{1}}\right]^{|p|}+c_{M_{0}}^{2} q^{0}(\lambda)\left[q_{2}(\lambda)\right]^{k_{0}-k_{1}}
\end{aligned}
$$

Soient $s(\lambda)=\left(|\lambda|^{-2 \mu+1 / 2}+|\lambda|^{-n-2 \mu+1 / 2}, s^{\prime}(\lambda)=\left(|\lambda|^{-2 \mu+1}+|\lambda|^{-(n+1)}\right)\right.$. On déduit facilement et i) et ii) la preuve de l'assertion a) pour $\eta=0$ et de iii) celle de l'assertion b) dans ce cas.

Il reste à prouver les deux assertions dans le cas général.

iv) Soit $0 \neq u \in \boldsymbol{R}^{n}$; on considère $u=\eta\|u\|^{-1} u$; comme on a les estimations (11) dans le tube $\xi+i(\theta(\lambda) / 2+\omega)\|\omega\| \leq \eta$, en utilisant la formule de Stokes, on trouve par exemple dans le cas iii) 


$$
\begin{aligned}
& \left|\int_{\boldsymbol{R}^{n}} \sum_{k_{1}=0}^{k_{0}}\left(\begin{array}{c}
k_{0} \\
k_{1}
\end{array}\right)(i \xi)^{\alpha-p-\beta-k_{1}} D_{u}^{p} D_{i}^{k_{0}-k_{1}} V_{\lambda}(u, \xi+i \theta(\lambda) 2) e^{i \xi \cdot x} d \xi\right| \\
& \leq \int_{\boldsymbol{R}^{n}} \sum_{k_{1}=0}^{k_{0}}\left(\begin{array}{c}
k_{0} \\
k_{1}
\end{array}\right)\left\|\xi+i u_{\eta}\right\|^{|\alpha-p-\beta|-k_{1}} \\
& \quad \times\left|D_{u}^{p} D_{i}^{k_{0}-k_{1}} V_{\lambda}\left(u, \xi+i\left(\theta(\lambda) 2+u_{\eta}\right)\right)\right| d \xi e^{-\eta\|x\|} .
\end{aligned}
$$

La technique développée au paragraphe précédent s'applique puisqu'on a les estimations (11); ce qui termine la preuve de a) et b).

Remarquons que si $Q_{\lambda}=Q_{\lambda}^{\prime} q_{1}^{\prime}(\lambda)$ où $q_{1}^{\prime}$ est un polynôme sans zéro, et $Q_{\lambda}^{\prime}$ satisfait les hypothèses du théorème, on obtient en principe une meilleure constante, $c_{1}$ voir $2^{\circ}$, en étudiant un opérateur qui est lié à $Q_{\lambda}$ :

$$
\left[q_{1}^{\prime}(\lambda)\right]^{-1} P_{\lambda}(\xi, D)=\widehat{T}(i \lambda \xi,-i \lambda) Q_{\lambda}^{\prime}(\xi, D)+\left[q_{1}^{\prime}(\lambda)\right]^{-1} B(\xi, \lambda, D) .
$$

D) Estimations de $G^{I}$

$\mathrm{D}_{1}$ ) Quelques estimations liées à $F_{\alpha, \beta, p}$.

i) $\eta=0$ et $2 \mu-|\alpha-p-\beta| \leq n$.

D'après (13) et (14)

$$
\begin{aligned}
\left|F_{\alpha, \beta, p}(u, x)\right| \leq & \|x\|^{-n+1 / 2}|q(\lambda)|^{-1 / 2} s(\lambda) \sum_{k=0}^{s} c_{M_{0}}^{1}\left[q^{0}(\lambda)\left(q_{2}(\lambda)\right)^{s-k}\right]^{|p|} \\
& +c_{M_{0}}^{2} q^{0}(\lambda)\left(q_{2}(\lambda)\right)^{s-k} \mathbf{1}_{\|x\|<a} \\
& +\|x\|^{-n-1}|\lambda|^{1 / 2} s(\lambda)|q(\lambda)|^{-1 / 2}\left(\sum_{k=0}^{k_{0}} c_{M_{0}}^{1}\left[q^{0}(\lambda)\left(q_{2}(\lambda)\right)^{s-k}\right]^{|p|}\right. \\
& \left.+c_{M_{0}}^{2} q^{0}(\lambda)\left(q_{2}(\lambda)\right)^{s-k}\right) \mathbf{1}_{\|x\| \geq a} .
\end{aligned}
$$

Il s'en suit que l'opérateur

$$
f \mapsto \int_{\boldsymbol{R}^{n}} F_{\alpha, \beta, p}(u, u-y) f(y) d y=F_{\alpha, \beta, p}(f)(u)
$$

est borné sur $L^{2}\left(\boldsymbol{R}^{n}\right)$ et sa norme ne dépasse pas l'intégrale du second membre ci-dessus:

$$
\begin{aligned}
& \left\|F_{\alpha, \beta, p}(f)\right\|_{2} \\
& \quad \leq s(\lambda)\left[1+|\lambda|^{1 / 2}\right]|q(\lambda)|^{-1 / 2} \sum_{k=0}^{k_{0}} c_{M_{0}}^{1}\left(q^{0}(\lambda)\left[q_{2}(\lambda)\right]^{k_{0}-k}\right)^{|p|} \\
& \quad+c_{M_{0}}^{2} q^{0}(\lambda)\left[q_{2}(\lambda)\right]^{k_{0}-k}\|f\|_{2} .
\end{aligned}
$$

ii) $\eta=0$ et $2 \mu-|\alpha-p-\beta| \geq n+1$.

On utilisera les inégalités (12) et (14) 


$$
\begin{aligned}
& \left|F_{\alpha, \beta, p}(u, x)\right| \\
& \leq\left\{\left|c q^{0}(\lambda) s_{1}(\lambda)\right||q(\lambda)|^{-1 / 2} \mathbb{H}_{\|x\| \leq a}+\|x\|^{-n-1}|q(\lambda)|^{-1 / 2} \mathbb{H}_{\|x\| \geq a}\right. \\
& \left.\quad \times \sum_{k=0}^{k_{0}} c_{M_{0}}^{1}\left(q^{0}(\lambda)\left[q_{2}(\lambda)\right]^{k_{0}-k}\right)^{|p|}+c_{M_{0}}^{2} q^{0}(\lambda)\left[q_{2}(\lambda)\right]^{k_{0}-k}\right\} \\
& \quad \times\left[s(\lambda)|\lambda|^{1 / 2}+s^{\prime}(\lambda)\right]
\end{aligned}
$$

et comme ci-dessus la norme de l'opérateur $f \mapsto F_{\alpha, \beta, p}(f)$ ne dépasse pas:

$$
\begin{aligned}
& \left\|F_{\alpha, \beta, p}(f)\right\|_{2} \\
& \quad \leq c\|f\|_{2}\left\{q^{0}(\lambda) s_{1}(\lambda)+\sum_{k=0}^{k_{0}} c_{M_{0}}^{\prime}\left(q^{0}(\lambda)\left[q_{2}(\lambda)\right]\right)^{k_{0}-k}\right. \\
& \left.\quad+c_{M_{0}}^{2} q^{0}(\lambda)\left[q_{2}(\lambda)\right]^{k_{0}-k}\right\}\left[s(\lambda)|\lambda|^{1 / 2}+s^{\prime}(\lambda)\right]|q(\lambda)|^{-1 / 2}
\end{aligned}
$$

iii) $\eta \neq 0$ par un choix approprié de la constante $a_{0}$, on remplace les constantes précédentes par:

$$
\begin{aligned}
& \left\|F_{\alpha, \beta, p}(f)\right\|_{2} \\
& \leq\|f\|_{2} e^{-\eta a_{0}}|q(\lambda)|^{-1 / 2} \sum_{k=0}^{k_{0}} c_{M_{0}}^{1}\left(q^{0}(\lambda)\left[q_{2}(\lambda)\right]^{k_{0}-k}\right)^{|\rho|} \\
& \quad+c_{M_{0}}^{2} q^{0}(\lambda)\left[q_{2}(\lambda)\right]^{k_{0}-k}\left\{s(\lambda)\left[1+|\lambda|^{1 / 2}\right\} w_{0}^{-1}\right.
\end{aligned}
$$

et

$$
\begin{aligned}
& \left\|F_{\alpha, \beta, p}(f)\right\| \\
& \leq \quad\left[s(\lambda)|\lambda|^{1 / 2}+s^{\prime}(\lambda)\right]|q(\lambda)|^{-1 / 2}\left\{q^{0}(\lambda) s_{1}(\lambda)\right. \\
& \left.\quad+\sum_{k=0}^{k_{0}} c_{M_{0}}^{1}\left(q^{0}(\lambda)\left[q_{2}(\lambda)\right]^{k_{0}-k}\right)^{|p|}+c_{M_{0}}^{2} q^{0}(\lambda)\left[q_{2}(\lambda)\right]^{k_{0}-k}\right\} e^{-\eta a_{0}} w_{0}^{-1}
\end{aligned}
$$

en utilisant l'estimation (15). Ici $w_{0}>0$ est un nombre choisi pour tenir compte de la taille de $\eta$.

$\left.\mathrm{D}_{2}\right) \quad$ Estimations des $\mathcal{E}_{\lambda, \beta}^{I, \alpha}=D_{t}^{\alpha} D_{x}^{\beta} \mathcal{E}_{\lambda}^{I}\left(t, t-y^{\prime}\right)$.

i) Pour simplifier l'exposé, on note $q_{\alpha}(\lambda)$ le coefficient de $D^{\alpha}$ dans $Q_{\lambda}$ et $b_{\alpha}(u, \lambda)$ celui de $D^{\alpha}$ dans $B(u, \lambda, D)$.

On remarque d'une part que:

$$
D_{t}^{a} \mathcal{E}_{\lambda}^{I}(t, t-y)=\sum_{0 \leq p \leq \alpha} c_{p, \alpha} D_{t}^{p} D_{x}^{a-p} \mathcal{E}_{\lambda}^{I}(t, t-y)
$$

où $c_{p, \alpha}$ sont certains constantes. Puisque $\mathcal{E}_{\lambda}^{I}$ est une solution fondamentale de $R_{\lambda}^{I}(u, D)$ on a l'égalité: 


$$
\begin{gathered}
R_{\lambda}^{I}(u, D) \mathcal{E}_{\lambda}^{I}(t, t-y)=I_{d}+\sum_{\alpha \neq 0} q_{\alpha}(\gamma) \sum_{0<p \leq \alpha} D_{t}^{p} D_{x}^{\alpha-p} \mathcal{E}_{\lambda}^{I}(t, t-y) c_{p, \alpha} \\
+\left(T^{I}\right)^{-1} \sum_{\alpha \neq 0} b_{\alpha}(u, \lambda) \sum_{0<p \leq \alpha} c_{p, \alpha} D_{t}^{p} D_{x}^{r-p} \mathcal{E}^{I}(t, t-y) .
\end{gathered}
$$

ii) D'après l'estimation (3), cette solution fondamentale de $R^{I}(u, D)$, est :

$$
\mathcal{E}_{\lambda}^{I} f(x)=e^{-\theta(\lambda) \cdot x / 2} \int_{\boldsymbol{R}^{n}} \frac{e^{i u \cdot x} \hat{f}(u+i \theta(\lambda) / 2)}{R_{\lambda}^{I}(x, u+i \theta(\lambda) / 2)} d u .
$$

Il est évident que

$$
\int_{\mathbb{R}^{n}}\left|\mathcal{E}_{\lambda}^{I} f(x)\right|^{2} e^{\theta(\lambda) \cdot x} d x \leq \sup _{u \in \mathbb{R}^{n}}\left|Q_{\lambda}(\xi, u+i \theta(\lambda) / 2)\right|^{-2} \int_{\mathbb{R}^{n}}|f(x)|^{2} e^{\theta(\lambda) \cdot x} d x
$$

On veut prouver que la norme de $G^{I}$ comme opérateur sur l'espace $L^{2}\left(\boldsymbol{R}^{n}, e^{\theta(\lambda) \cdot x} d x\right)$ ne dépasse pas 1 .

Alors

$$
\begin{aligned}
D_{t}^{p} D_{x}^{\alpha-p} \mathcal{E}_{\lambda}^{I} f(y) \\
=\int_{\mathbb{R}^{n}} D_{t}^{p} V_{\lambda}(x, \xi+i \theta(\lambda) / 2)(\xi+i \theta(\lambda) / 2)^{\alpha-p} \\
\quad \times \widehat{f}(\xi+i \theta(\lambda) / 2) e^{i x \cdot(\xi+i \theta(\lambda) / 2)} d \xi
\end{aligned}
$$

et pour estimer la norme de $\mathcal{E}_{\lambda, \alpha-p}^{I, \alpha}$ comme opérateur sur $L^{2}\left(\mathbb{R}^{n}, e^{\theta(\lambda) \cdot x} d x\right)$ il suffit alors d'estimer la norme de l'opérateur $f \mapsto F_{\alpha, p}(f)$, qui à été etudiée au paragraphe $D_{1}$.

$\left.\mathrm{D}_{3}\right)$ Remarquons enfin qu'un calcul analogue à celui de page 440 montre que:

$$
\left|\left(T^{I}\right)^{-1}(u, \lambda) b_{\alpha}(u, \lambda)\right| \leq\left|q_{0}(\lambda)\right|^{-\beta} \sum_{p, r, s} b_{p, r, s}|\lambda|^{s+r}=c_{4}(\lambda, \beta) .
$$

On aura besoin de cette estimation pour prouver le théorème III.

E) Fin de la preuve du théorème.

$1^{\circ}$ La norme de l'opérateur $G_{\lambda}^{I}$ sur $L^{2}\left(\boldsymbol{R}^{n}, e^{\theta(\lambda) \cdot x} d x\right)$ ne dépasse pas la somme:

$$
\left\|G_{\lambda}^{I}\right\| \leq \sum_{\substack{0<p<\alpha \\|\alpha| \leq 2 \mu}}\left\|F_{\alpha, 0, p}\right\|\left[1+c_{4}(\lambda, \beta)\right]
$$

d'après $D_{2}$ ) i) et $D_{3}$ ).

D'après les inégalités (15) et (17), sous l'hypothèse $\eta=0$ 


$$
\begin{aligned}
\left\|G_{\lambda}^{I}\right\| \leq & \sum_{0<|p| \leq 2 \mu} c\left[1+c_{4}(\lambda, \beta)\right]\left[s(\lambda)+s^{\prime}(\lambda)\right]|q(\lambda)|^{-1 / 2} \\
& \times\left\{q^{0}(\lambda) s_{1}(\lambda)+\sum_{i=0}^{n+1} c_{M_{0}}^{1}\left(q^{0}(\lambda)\left[q_{2}(\lambda)\right]^{n+1-k}\right)^{|p|}+c_{M_{0}}^{2} q^{0}(\lambda)\left[q_{2}(\lambda)\right]^{n+1-k}\right\} \\
\leq & c\left[1+c_{4}(\lambda, \beta)\right]|q(\lambda)|^{-1 / 2}\left\{q^{0}(\lambda) s_{1}(\lambda)+\left[q^{0}(\lambda)\left[q_{2}(\lambda)\right]^{n+1}\right]^{2 \mu}\right. \\
& \left.+q^{0}(\lambda)\left[q_{2}(\lambda)\right]^{n+1}\right\}\left[s(\lambda)+s^{\prime}(\lambda)\right] .
\end{aligned}
$$

Puis que $c_{4}(\lambda, \beta)$ est bornée, comme il en est ainsi de $c(\lambda)$.

$$
\begin{aligned}
\left\|G^{I}\right\|=O\left(|q(\lambda)|^{-1 / 2}\right) & \left\{q^{0}(\lambda) s_{1}(\lambda)+\left(q^{0}(\lambda)\left[q_{2}(\lambda)\right]^{n+1}\right)^{2 \mu}+q^{0}(\lambda)\left[q_{2}(\lambda)\right]^{n+1}\right\} \\
& \left.\times\left(s(\lambda)+s^{\prime}(\lambda)\right)\right) .
\end{aligned}
$$

Elle sera plus petite que un si la condition i) de la page 402 est remplie pour un certain entier $L_{T}$.

Dans ce cas on trouvera une solution fondamentale $\widetilde{E}_{\lambda}^{I}$ de $R_{\lambda}^{I}(u, D)$ qui est bornée sur $L^{2}\left(\mathbb{R}^{n}, e^{\theta(\lambda) \cdot x} d x\right)$ et l'on peut s'arranger de telle sorte que sa norme soit plus petite qu'une constante donné en choisissant $C\left(\varepsilon, \varepsilon^{\prime}\right.$, $\theta, \eta, \mu, m)$ assez petite.

L'application $f \mapsto \widetilde{E}_{\lambda}^{\prime I}(f)=\widetilde{E}_{\lambda}^{I}\left(\left(T^{I}\right)^{-1} f\right)$ est bornée sur $L^{2}\left(\mathbb{R}^{n}\right.$, $\left.e^{\theta(\lambda) \cdot x} d x\right)$; c'est une solution fondamentale de $P_{\lambda, I}^{0}$.

$2^{\circ}$ On va définir une solution fondamentale de $P_{k}(\xi, D)$. Soit $U \in$ $C_{c}^{\infty}\left(\mathbb{R}^{n}\right)$ à support disjoint des axes de coordonnées. Pour tout $I \subset J$, on pose: $E_{\lambda}^{I}(U)=E_{\lambda}^{\prime I}\left(U_{I}\right) \circ \psi_{I}^{-1}$ et

$$
E_{\lambda}^{1} U=\sum_{I \subset J} E_{\lambda}^{I}(U)
$$

on vérifie facilement que:

$$
\left.\int_{\mathbb{R}^{n}}\left|E_{\lambda}^{1} U(x)\right|^{2}\left|x_{J} !^{-[1-\theta(\lambda)]} d x \leq-\frac{c}{q_{1}(\lambda) m+\beta} \int_{\mathbb{R}^{n}}\right| u(x)\right|^{2}\left|x_{J}\right|^{[1-\theta(\lambda)]} d x
$$

on vérifie aussi comme à la proposition (3) que $E_{\lambda}^{1}$ est une solution de $P_{\lambda}(\xi, D)$. Soit $H^{\theta}$, resp. $H^{-\theta}$ l'opérateur de convolution dont la transformée de Fourier pour chaque $\lambda$ est l'opérateur de multiplication par $|\lambda|^{(1-\theta(\lambda)) / 2}\left|x_{J}\right|^{(1-\theta(\lambda)) / 2}$, resp. $|\lambda|^{-(1-\theta(\lambda)) / 2}\left|x_{J}\right|^{-(1-\theta(\lambda)) / 2}$. On voit comme à la page 421 qu'il existe un opérateur de convolution $E^{\prime}$ borné sur $L^{2}\left(H_{n}\right)$ tel que si l'on pose $E^{1}=H^{\theta} E^{\prime}$

$$
P E^{1}=I I^{\theta} \text {. }
$$

Pour terminer la preuve du théorème il suffit d'utiliser comme en (40) 
le lemme suivant:

Lemme 9. On suppose que $\varepsilon_{i}<\theta_{i}(\lambda)<2-\varepsilon_{i}^{\prime}, 0<\varepsilon_{i}, 0<\varepsilon_{i}^{\prime}<1$

$$
\begin{aligned}
& \left\|H^{\theta} f\right\|_{L^{2}\left(H_{n}\right)}^{2} \leq c \sum_{|s| \leq 2 n}\left(\int_{\mathbb{R}^{n+1}}\left(\int_{\boldsymbol{R}^{n}}\left|D^{s} f(x, y, z)\right|^{r_{\theta}} d y\right)^{2 / r_{\theta}^{\prime}} d x d z\right)^{1 / 2} \\
& \left\|H^{-\theta} f\right\|_{L^{2}\left(H_{n}\right)} \leq c \sum_{|s| \leq 2 n}\left(\int_{\boldsymbol{R}^{n+1}}\left(\int_{\boldsymbol{R}^{n}}\left|D^{s} f(x, y, z)\right|^{r} d y\right)^{2 / r} d y d z\right)^{1 / 2}
\end{aligned}
$$

où

$$
1 / r_{\theta}^{\prime}=\sup _{i}\left(1 / 2+\left(1-\varepsilon_{i}^{\prime}\right) / 2\right) ; 1 / r_{\theta}=\sup _{i}\left(1 / 2+\left(1-\varepsilon_{i}\right) / 2\right)
$$

La preuve de ce lemme est tout à fait analogue à celle de la proposition 6; on laisse au lecteur.

$E=E^{1} * H^{-\theta}$ est une solution fondamentale de $P$, comme on le vérifie immédiatement. Signalons que si $\theta_{i}(\lambda)=1 / 2, E$ est un opérateur de convolution borné sur $L^{2}\left(H_{n}\right)$.

Ceci termine la preuve du théorème III.

Ennonçons un corollaire du théorème III.

Corollaire 10. Soit $P(D)=Q_{1}(D) Q_{2}(D)$ où $Q_{1}(D)$ est de la forme décrite dans le théorème III et $Q_{2}(D)$ est comme au théorème $I$ alors $P(D)$ est localement résoluble et surjectif sur $C^{\infty}\left(H_{n}\right)$.

La preuve de ce corollaire est sensiblement la même qu'à la page 423.

Il est plus facile en partant des estimations à priori de prouver que $E^{*}$ posséde une solution fondamentale sous l'hypothèse que $c_{1}(\lambda)$ n'est pas trop grand.

\section{Bibliographie}

[1] Chang, W., Global solvability of invariant differential operators, Ph. D. Thesis M. I. T., 1978.

[2] Fefferman, Ch. et Stein, E. M., $H^{p}$ spaces of several variables, Acta. Mathematica 129 (1972), 137-193. 
[3] Helgason, S., invariant differential equations on homogeneous manifolds, Bull. of A.M.S. 83, No. 5, Sept. 1977.

[4] Lion, G., Hypoellipticité et résolubilité d'opérateurs différentiels sur des groups nilpotents de rang 2, C. R. Acad. Sc. 290, Serie A. fevrier 1980, 271-274.

[5] Treves, F., Linear partial differential equations with constant coefficients, Gordon and Beach Science Publishers, New York-London. 
